\title{
WestVirginiaUniversity
}

THE RESEARCH REPOSITORY @ WVU

Graduate Theses, Dissertations, and Problem Reports

2011

\section{Group Colorability and Hamiltonian Properties of Graphs}

Hao Li

West Virginia University

Follow this and additional works at: https://researchrepository.wvu.edu/etd

\section{Recommended Citation}

Li, Hao, "Group Colorability and Hamiltonian Properties of Graphs" (2011). Graduate Theses,

Dissertations, and Problem Reports. 3374.

https://researchrepository.wvu.edu/etd/3374

This Dissertation is protected by copyright and/or related rights. It has been brought to you by the The Research Repository @ WVU with permission from the rights-holder(s). You are free to use this Dissertation in any way that is permitted by the copyright and related rights legislation that applies to your use. For other uses you must obtain permission from the rights-holder(s) directly, unless additional rights are indicated by a Creative Commons license in the record and/ or on the work itself. This Dissertation has been accepted for inclusion in WVU Graduate Theses, Dissertations, and Problem Reports collection by an authorized administrator of The Research Repository @ WVU.

For more information, please contact researchrepository@mail.wvu.edu. 


\title{
Group Colorability and Hamiltonian Properties of Graphs
}

\author{
Hao Li \\ Dissertation submitted to the \\ Eberly College of Arts and Sciences \\ at West Virginia University \\ in partial fulfillment of the requirements \\ for the degree of \\ Doctor of Philosophy \\ in \\ Mathematics \\ Hong-Jian Lai, Ph.D., Chair \\ John Goldwasser, Ph.D. \\ K. Subramani, Ph.D. \\ Jerzy Wojciechowski, Ph.D. \\ Cun-Quan Zhang, Ph.D. \\ Department of Mathematics \\ Morgantown, West Virginia \\ 2011
}

Keywords: Group coloring, Multigraph, Cartesian product, Hamiltonian, Collapsible, Reduction, P3D, subdivision

Copyright 2011 Hao Li 


\section{ABSTRACT \\ Group Colorability and Hamiltonian Properties of Graphs}

\section{Hao Li}

The research of my dissertation was motivated by the conjecture of Thomassen that every 4-connected line graph is hamiltonian and by the conjecture of Matthews and Sumner that every 4-connected claw-free graph is hamiltonian. Towards the hamiltonian line graph problem, we proved that every 3-edge-connected, essentially 4-edge-connected graph $G$ has a spanning eulerian subgraph, if for every pair of adjacent vertices $u$ and $v, d_{G}(u)+d_{G}(v) \geq 9$. A straight forward corollary is that every 4-connected, essentially 6 -connected line graph with minimum degree at least 7 is hamiltonian.

We also investigate graphs $G$ such that the line graph $L(G)$ is hamiltonian connected when $L(G)$ is 4-connected. Ryjáček and Vrána recently further conjectured that every 4-connected line graph is hamiltonian-connected. In 2001, Kriesell proved that every 4connected line graph of a claw free graph is hamiltonian connected. Recently, Lai et al showed that every 4-connected line graph of a quasi claw free graph is hamiltonian connected, and that every 4-connected line graph of an almost claw free graph is hamiltonian connected. In 2009, Broersma and Vumer discovered the $P_{3}$-dominating (P3D) graphs as a superfamily that properly contains all quasi claw free graphs, and in particular, all claw-free graphs. Here we prove that every 4-connected line graph of a P3D graph is hamiltonian connected, which extends several former results in this area.

R. Gould [15] asked what natural graph properties of $G$ and $H$ are sufficient to imply that the product of $G$ and $H$ is hamiltonian. we first investigate the sufficient and necessary conditions for $G \times H$ being hamiltonian or traceable when $G$ is a hamiltonian graph and $H$ is a tree. Then we further investigate sufficient and necessary conditions for $G \times H$ being hamiltonian connected, or edge-pancyclic, or pan-connected.

The problem of group colorings of graphs is also investigated in this dissertation. Group coloring was first introduced by Jeager et al. [21]. They introduced a concept of 
group connectivity as a generalization of nowhere-zero flows. They also introduced group coloring as a dual concept to group connectivity. Prior research on group chromatic number was restricted to simple graphs, and considered only Abelian groups in the definition of $\chi_{g}(G)$. The behavior of group coloring for multigraphs is different to that of simple graphs. Thus we extend the definition of group coloring by considering general groups (both Abelian groups and non-Abelian groups), and investigate the properties of $\chi_{g}$ for multigraphs by proving an analogue to Brooks' Theorem. 


\section{Acknowledgements}

First and foremost, I am most indebted to my supervisor, Dr. Hong-Jian Lai, for his continued encouragement and support over these last few years. It is a pleasure to work under his supervision. Without him, this paper could not have come about.

I would like to take this opportunity to thank Dr. Mei Lu at Tsinghua University, who brought me to the research field of graph theory.

I would also like to thank my other committee members: Dr. John Goldwasser, Dr. K. Subramani, Dr. Jerzy Wojciechowski, and Dr. Cun-Quan Zhang, for their help during my studies.

And finally, I would like to thank the Department of Mathematics and Eberly College of Arts and Sciences at West Virginia University for providing me with an excellent study environment and support during my study as a graduate student. 


\section{DEDICATION}

To

my father Jiawang Li , my mother Xiaomei Zhou

and

my wife $\underline{\text { Xin Gao }}$ 


\section{Contents}

1 Preliminaries 1

1.1 Notation and Terminology . . . . . . . . . . . . . . . . . . . . 1

1.2 Catlin's Reduction Method . . . . . . . . . . . . . . . . 5 5

1.3 Main Results . . . . . . . . . . . . . . . . . . . . . . . 7

2 Hamiltonian properties in Cartesian product $\quad 9$

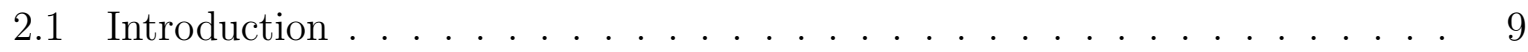

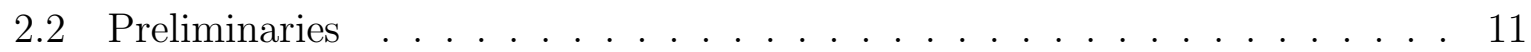

2.3 Hamiltonian circuit in $G \times T \ldots \ldots \ldots \ldots \ldots$

2.4 Hamiltonian path in $G \times T \ldots \ldots \ldots \ldots$

2.5 Hamiltonian connected Cartesian product . . . . . . . . . . . . . 21

2.6 Weakly edge-pancyclic Cartesian product . . . . . . . . . . . . . 22

2.7 Pan-connected Cartesian product . . . . . . . . . . . . . 24

3 On 3-edge-connected Supereulerian Graphs 27 
3.1 Introduction . . . . . . . . . . . . . . . 27

3.2 Preliminary . . . . . . . . . . . . . . . . . . . . . . . . . . 29

3.3 Proofs of Theorems . . . . . . . . . . . . . . . . . . 31

4 4-connected line graph of a $P 3 D$ graph 35

4.1 Introduction . . . . . . . . . . . . . . . . . 35

4.2 Preliminaries . . . . . . . . . . . . . . . . . . . . 37

4.3 Local Graphs of Small Degree Vertices in a P3D Graph . . . . . . . . . . . 46

4.4 Hamiltonian Connectedness of $P_{3}$-dominating Graphs . . . . . . . . . . 57

$5 \quad$ Group Colorability of Multigraphs $\quad 78$

5.1 Introduction . . . . . . . . . . . . . . . . . 78

5.2 Properties of Group Coloring . . . . . . . . . . . . . . . . 80

5.3 Degeneration Method . . . . . . . . . . . . . . . . . . 82

5.4 Group Chromatic Number of Multigraphs . . . . . . . . . . . . . 83 


\section{Chapter 1}

\section{Preliminaries}

\subsection{Notation and Terminology}

We use [4] for terminology and notations not defined here. All graphs in this thesis are finite and undirected. A graph with at most one vertex is called a trivial graph. Let $G$ be a graph. We use $V(G)$ and $E(G)$ to denote the set of vertices and the set of edges of $G$, respectively. Two vertices $u, v$ are adjacent if $u v \in E(G)$. For a vertex $v \in V(G)$, the number of edges incident with $v$ is called the degree of $v$ in $G$, and is denoted by $d_{G}(v)$ or $d(v)$. We use $N_{G}(v)$ and $E_{G}(v)$ to denote the set of vertices adjacent to $v$ and the set of edges incident with $v$, respectively. We use $\Delta(G)$ and $\delta(G)$ to denote the maximum and minimum degree of $G$, respectively. For each integer $i=0,1,2, \cdots$, we let $D_{i}(G)=\left\{v \in V(G): d_{G}(v)=i\right\}$. A graph is called a $k$-regular graph if every vertex is of degree $k$.

A walk (of length $k$ ) in $G$ is a non-empty alternating sequence $v_{0} e_{0} v_{1} e_{1} \ldots e_{k-1} v_{k}$ of vertices and edges in $G$ such that $e_{i}=v_{i} v_{i+1}$ for all $0 \leq i \leq k-1$. If the edges in a walk are distinct, it defines a trail in $G$. If the vertices in a walk are distinct, it defines a path in $G$. We use $P_{k}$ to denotes a path with $k$ vertices. A graph is connected if any two of its vertices are linked by a path. A maximal connected subgraph of a graph is called a component, and let $c(G)$ denote the number of components of $G$. If any two 
vertices are adjacent in $G$, then $G$ is called a complete graph. A complete graph with $n$ vertices is denoted by $K_{n}$. A nontrivial 2-regular connected graph is called a circuit, and a $k$-circuit, denoted by $C_{k}$, is a circuit of $k$ vertices. A star, denoted by $K_{1, j}(j \geq 0)$, is a graph with $j+1$ vertices and $j$ edges, in which one vertex is adjacent with other $j$ vertices. The center of a $K_{1, j}$ is the vertex of degree $j$. We use $H \subseteq G$ to denote the fact that $H$ is a subgraph of $G$.

For a connected graph $G$, a vertex cut of $G$ is a subset $V^{\prime}$ of $V(G)$ such that $G-V^{\prime}$ is disconnected. A $k$-vertex cut is a vertex cut of $k$ elements. A complete graph has no vertex cut; in fact, the only graphs that do not have vertex cuts are those that contain complete graphs as spanning subgraphs. $G$ is called $k$-connected (for some positive integer $k$ ) if $G$ has no $i$-vertex cut for all $1 \leq i \leq k-1$. The greatest integer $k$ such that $G$ is $k$-connected is the connectivity $\kappa(G)$ of $G$. In particular, $\kappa\left(K_{n}\right)=n-1$. Similarly, an edge cut of $G$ is a subset $E^{\prime}$ of $E(G)$ such that $G-E^{\prime}$ is disconnected. $G$ is called $l$-edge-connected (for some positive integer $l$ ) if $G$ has no $i$-edge cut for all $1 \leq i \leq l-1$. The greatest integer $l$ such that $G$ is $l$-edge-connected is the edgeconnectivity $\kappa^{\prime}(G)$ of $G$. An edge cut $X$ of $G$ is peripheral if for some $v \in V(G)$, $X=E_{G}(v)$; and is essential if each side of $G-X$ has an edge. A graph $G$ is essentially $k$-edge-connected if $|E(G)| \geq k+1$ and if for every $E_{0} \subseteq E(G)$ with $\left|E_{0}\right|<k, G-E_{0}$ has exactly one component $H$ with $E(H) \neq \emptyset$.

Let $A, B$ be the subsets of $V(G)$ with $A \cap B=\emptyset$. An edge $x y \in E(G)$ is called an $A$-B edge if $x \in A$ and $y \in B$. The set of all $A$ - $B$ edges in $G$ is denoted by $[A, B]_{G}$. Instead of $[\{u\}, B]_{G}$ and $[\{u\},\{v\}]_{G}$, we simply write $[u, B]_{G}$ and $[u, v]_{G}$. The number of edges in $[A, B]_{G}$ is denoted by $\|A, B\|_{G}$. For two vertex-disjoint subgraphs $H_{1}, H_{2}$ of $G$, we write $\left[H_{1}, H_{2}\right]_{G}$ for $\left[V\left(H_{1}\right), V\left(H_{2}\right)\right]_{G}$. For simplicity, we use $[A, B]$ and $\|A, B\|$ for $[A, B]_{G}$ and $\|A, B\|_{G}$, respectively.

For $V_{1} \subseteq V(G)$, let $G\left[V_{1}\right]$ denote the subgraph induced by $V_{1}$. Let $H$ be a subgraph of $G$. For $E_{1} \subseteq E(H), E_{2} \cap E(H)=\emptyset, V_{1} \subseteq V(H)$, and $V_{2} \cap V(H)=\emptyset$, the subgraphs induced by $E(H)-E_{1}, E(H) \cup E_{2}, V(H)-V_{1}, V(H) \cup V_{2}$ are denoted by $H-E_{1}, H+$ $E_{2}, H-V_{1}, H+V_{2}$, respectively. For $E_{1}=\left\{e_{1}\right\}, E_{2}=\left\{e_{2}\right\}, V_{1}=\left\{v_{1}\right\}, V_{2}=\left\{v_{2}\right\}$, we abbreviate them to $H-e_{1}, H+e_{2}, H-v_{1}$, and $H+v_{2}$. 
Let $X \subseteq E(G)$. The contraction $G / X$ is the graph obtained from $G$ by identifying the two ends of each edge in $X$ and then deleting the resulting loops. For convenience, we use $G / e$ for $G /\{e\}$ and $G / \emptyset=G$; and if $H$ is a subgraph of $G$, we write $G / H$ for $G / E(H)$. Note that even if $G$ is a simple graph, contracting some edges of $G$ may result in a graph with multiple edges. If $K$ is a connected subgraph of $G$, and if $v_{K}$ is the vertex in $G / K$ onto which $K$ is contracted, then $K$ is called the pre-image of $v_{K}$, and is denoted by $P I\left(v_{K}\right)$. A vertex $v$ in a contraction of $G$ is a nontrivial vertex if $P I(v)$ has at least one edge.

A subgraph $H$ of $G$ is spanning if $V(H)=V(G)$, and a spanning path (or circuit) of $G$ is sometimes called a hamiltonian path (or circuit) of $G$. A graph is traceable if it contains a hamiltonian path, and hamiltonian if it contains a hamiltonian circuit. For a pair of distinct vertices $u$ and $v$ in $G$, a $(u, v)$-path is a path linking $u$ and $v$. The distance between $u$ and $v$, denoted by $\operatorname{dist}_{G}(u, v)$, is the length of a shortest $(u, v)$-path in $G$. $G$ is hamiltonian connected if for every pair of distinct vertices $u$ and $v$, there is a hamiltonian $(u, v)$-path. $G$ is edge-pancyclic, if every edge lies on a circuit of length $l$ for all $l=3,4, \cdots,|V(G)| . G$ is pan-connected if for every pair of distinct vertices $u$ and $v$, there is a $(u, v)$-path of length $l$ for all $l=\operatorname{dist}_{G}(u, v), \cdots, n-1$.

The line graph of a graph $G$, denoted by $L(G)$, has $E(G)$ as its vertex set, where two vertices in $L(G)$ are adjacent if and only if the corresponding edges in $G$ are adjacent. For a graph $G$, an induced subgraph $H$ isomorphic to $K_{1,3}$ is called a claw of $G$, and the only vertex of degree 3 of $H$ is the center of the claw. A graph $G$ is claw free if it does not contain a claw. Beineke ([2]) and Robertson ([33] and [17]) showed that every line graph is also a claw-free graph.

Proposition 1.1.1 Let $G$ be a nontrivial simple graph. Then $L(G)$ is complete if and only if $G$ is a $K_{3}$ or a $K_{1, n}$ for an integer $n \geq 1$.

Proof. The line graph $L(G)$ is complete if and only if any two edges in $G$ are incident. If $|E(G)|=1, G=K_{2}=K_{1,1}$; if $|E(G)|=2, G=P_{2}=K_{1,2}$; if $|E(G)|=3, G=K_{3}$ or $G=K_{1,3} ;$ if $|E(G)| \geq 4, G=K_{1, n}$.

A subgraph $H$ of a graph $G$ is dominating if $E(G-V(H))=\emptyset$. Let $O(G)$ denote the 
set of odd degree vertices of $G$. A graph $G$ is eulerian if $O(G)=\emptyset$ and $G$ is connected. Note that the graph $K_{1}$ is also eulerian.

A well known relationship between dominating Eulerian subgraphs in $G$ and hamiltonian circuits in $L(G)$ is given by Harary and Nash-Williams.

Theorem 1.1.2 (Harary and Nash-Williams, [18]) Let $G$ be a graph with $|E(G)| \geq 3$. Then $L(G)$ is hamiltonian if and only if $G$ has a dominating eulerian subgraph.

The following follows by a similar argument in the proof of Theorem 1.1.2.

Proposition 1.1.3 Let $G$ be a graph with $|E(G)| \geq 3$. Then $L(G)$ is hamiltonian connected if and only if for any pair of edges $e^{\prime}, e^{\prime \prime} \in E(G), G$ has a dominating $\left(e^{\prime}, e^{\prime \prime}\right)$-trail.

Let $G$ be a connected, essentially 3-edge-connected graph such that $L(G)$ is not a complete graph. The core of the graph $G$, denoted by $G_{0}$, is obtained by applying the following two operations repeatedly:

Operation 1. Delete a vertex of degree 1.

Operation 2. For each vertex $y$ of degree 2 with $\Theta(y)=\{x y, y z\}$, contract exactly one edge in $\Theta(y)$. This amounts to deleting the vertex $y$ for the path $x y z$ in $G$ with $d_{G}(y)=2$ and adding the new edge $x z$.

Shao [38] proved Theorem 1.1.4 (a)-(c). In a similar way as Theorem 1.1.4 (c), one can prove Theorem 1.1.4 (d).

Theorem 1.1.4 (Shao, [38]) Let $G$ be a connected and essentially 3-edge-connected graph and let $G_{0}$ be the core of graph $G$, then each of the following holds:

(a) $G_{0}$ is nontrivial and $\delta\left(G_{0}\right) \geq \kappa^{\prime}\left(G_{0}\right) \geq 3$;

(b) $G_{0}$ is well defined; 
(c) If $G_{0}$ has a spanning eulerian subgraph, then $G$ has a dominating eulerian subgraph;

(d) If $G_{0}$ has a dominating eulerian subgraph containing all nontrivial vertices and both endvertices of each nontrivial edges, then $G$ has a dominating eulerian subgraph.

\subsection{Catlin's Reduction Method}

In [7] Catlin defined collapsible graphs. Given a subset $R$ of $V(G)$, a subgraph $\Gamma$ of $G$ is called an $R$-subgraph if both $O(\Gamma)=R$ and $G-E(\Gamma)$ is connected. A graph $G$ is collapsible if for any even subset $R$ of $V(G), G$ has an $R$-subgraph. In particular, $K_{1}$ is collapsible.

For collapsible graphs, there is another equivalent definition: a graph $G$ is collapsible if for any even subset $X$ of $V(G), G$ has a spanning connected subgraph $R_{X}$ of $G$ such that $O\left(R_{X}\right)=X$.

Catlin [7] showed that every graph $G$ has a unique collection of pairwise vertexdisjoint maximal collapsible subgraphs $H_{1}, H_{2}, \cdots, H_{k}$ such that $\bigcup_{i=1}^{k} V\left(H_{i}\right)=V(G)$. The $c$-reduction of $G$ is the graph obtained from $G$ by successively contracting $H_{1}, H_{2}, \cdots$, $H_{k}$. This contraction is called the $c$-contraction. If $H_{i}$ is $c$-contracted to $v_{i}$, then $H_{i}$ is called the $c$-preimage of $v_{i}$, and denoted by $\mathcal{C P}\left(v_{i}\right)$. Note that if $G$ has an $O(G)$-subgraph $\Gamma$, then $G-E(\Gamma)$ is a spanning eulerian subgraph of $G$. Therefore, every collapsible graph is supereulerian.

Theorem 1.2.1 Let $G$ be a graph and let $H$ be a collapsible subgraph of $G$. Let $v_{H}$ denote the vertex onto which $H$ is contracted in $G / H$. Each of the following holds.

(i) (Catlin, Theorem 3 of [7]) $G$ is collapsible if and only if $G / H$ is collapsible. In particular, $G$ is collapsible if and only if the reduction of $G$ is $K_{1}$.

(ii) (Catlin, Theorem 8 of [7]) 2-circuits and 3-circuits are collapsible.

(iii) ([27]) If $G$ is collapsible, then for any pair of vertices $u, v \in V(G), G$ has a spanning $(u, v)$-trail. 
(iv) ([27]) For vertices $u, v \in V(G / H)-\left\{v_{H}\right\}$, if $G / H$ has a spanning $(u, v)$-trail, then $G$ has a spanning $(u, v)$-trail.

(v)(Catlin, Theorem 5 of [7])Any subgraph of a reduced graph is reduced.

(vi) ([r]) If $G$ is collapsible, and if $e \in E(G)$, then $G / e$ is also collapsible.

(vii) (Lemma 1 of [8]) $K_{3,3}-e$ is collapsible.

(viii) (Catlin, Theorem 3 of [7]) Let $H$ be a collapsible subgraph of $G$. Then $G$ is supereulerian if and only if $G / H$ is supereulerian.

Let $\tau(G)$ denote the maximum number of edge-disjoint spanning trees of $G$. We assume that $\tau\left(K_{1}\right)=\infty$. Catlin showed the relationship between $\tau(G)$ and the edgeconnectivity $\kappa^{\prime}(G)$.

Theorem 1.2.2 Let $G$ be a graph, $H$ be a subgraph of $G$, and $k>0$ be an integer.

(i) (Catlin, Theorem 5.1 of [8]) $\kappa^{\prime}(G) \geq 2 k$ if and only if for any edge subset $X \subseteq E(G)$ with $|X| \leq k, \tau(G-X) \geq k$.

(ii) If $\tau(H) \geq k$ and if $\tau(G / H) \geq k$, then $\tau(G) \geq k$.

Theorem 1.2.3 (Catlin and Lai, Theorem 4 of [12]) Let $G$ be a graph with $\tau(G) \geq 2$ and let $e^{\prime}, e^{\prime \prime} \in E(G)$. Then $G$ has a spanning $\left(e^{\prime}, e^{\prime \prime}\right)$-trail if and only if $\left\{e^{\prime}, e^{\prime \prime}\right\}$ is not an essential edge cut of $G$.

We define $F(G)$ be the minimum number of additional edges that must be added to $G$ such that the resulting graph has two edge-disjoint spanning trees.

Theorem 1.2.4 Let $G$ be a graph.

(i) (Catlin, Han and Lai, Lemma 2.3 of [10]) If for any $H \subset G$ with $|V(H)|<|V(G)|$, $H$ is reduced, and if $|V(G)| \geq 3$, then $F(G)=2|V(G)|-|E(G)|-2$.

(ii) (Catlin, Theorem 7 of [r]) If $F(G) \leq 1$, then $G$ is collapsible if and only if $\kappa^{\prime}(G) \geq 2$.

(iii) (Catlin, Han and Lai, Theorem 1.3 of [10]) Let $G$ be a connected graph and $t$ an integer. If $F(G) \leq 2$, then $G$ is collapsible if and only if the c-reduction of $G$ is not a member in $\left\{K_{2}\right\} \cup\left\{K_{2, t}: t \geq 1\right\}$. 
(iv) (Catlin, Theorem 2 of [7]) If $F(G)=0$, then $G$ is collapsible and hence $G$ is supereulerian.

Theorem 1.2.5 (Lai, [24]) Let $G$ be a 2-connected graph with $\delta(G) \geq 3$. If every edge of $G$ is in a circuit of length at most 4 , then $G$ is collapsible.

It is known that all complete graphs of order at least 3 are collapsible and any circuit of length at least 4 is not collapsible. If $G$ contains a 4 -circuit $C=u v z w u$ with a partition $\pi=\langle\{u, z\},\{v, w\}\rangle$, then we can follow Catlin [6] and define $G / \pi(C)$ to be the graph obtained from $G-E(C)$ by identifying $u$ and $z$ to form a new vertex $x$, identifying $v$ and $w$ to form a new vertex $y$, and adding an edge $e_{\pi}=x y$.

Theorem 1.2.6 (Catlin, [6]) Let $G$ be a graph containing a 4-circuit $C$ and let $G / \pi(C)$ be defined as above. Each of the following holds:

(i) If $G / \pi(C)$ is collapsible, then $G$ is collapsible;

(ii) If $G / \pi(C)$ has a spanning eulerian subgraph, then $G$ has a spanning eulerian subgraph, i.e., if $G / \pi(C)$ is supereulerian, then $G$ is supereulerian.

\subsection{Main Results}

In the consequent chapters, we will present the following main results.

(1) R. Gould [15] asked what natural graph properties of $G$ and $H$ are sufficient to imply that the product of $G$ and $H$ is hamiltonian. we first investigate the sufficient and necessary conditions for $G \times H$ being hamiltonian or traceable when $G$ is a hamiltonian graph and $H$ is a tree. Then we further investigate sufficient and necessary conditions for $G \times H$ being hamiltonian connected, or edge-pancyclic, or pan-connected for more generic graphs $G$ and $H$.

(2) The supereulerian graph problem, raised by Boesch, Suffel, and Tindell [3], is to determine when a graph has a spanning eulerian subgraph. Pulleyblank showed that such 
a decision problem, even when restricted to planar graphs, is NP-complete. Jaeger [20] and Catlin [7] independently showed that every 4-edge-connected graph has a spanning eulerian subgraph. In 1992, Zhan [44] showed that every 3-edge-connected, essentially 7edge-connected graph has a spanning eulerian subgraph. It has been conjectured in 1995 that every 3-edge-connected, essentially 5-edge-connected graph has a spanning eulerian subgraph. Here we show that if $G$ is a 3-edge-connected, essentially 4-edge-connected graph and if for every pair of adjacent vertices $u$ and $v, d_{G}(u)+d_{G}(v) \geq 9$, then $G$ has a spanning eulerian subgraph.

(3) Mathews and Sumner [31], and Tommasen [40] conjectured that every 4-connected line graph is hamiltonian. We investigate graphs $G$ such that the line graph $L(G)$ is hamiltonian connected when $L(G)$ is 4-connected. Ryjáček and Vrána [37] recently further conjectured that every 4-connected line graph is hamiltonian-connected. In 2001, Kriesell [23] proved that every 4-connected line graph of a claw free graph is hamiltonian connected. Recently, Lai et al [27] showed that every 4-connected line graph of a quasi claw free graph is hamiltonian connected, and that every 4-connected line graph of an almost claw free graph is hamiltonian connected. In 2009, Broersma and Vumer [5] discovered the $P_{3}$-dominating (P3D) graphs as a superfamily that properly contains all quasi claw free graphs, and in particular, all claw-free graphs. Here we prove that every 4-connected line graph of a P3D graph is hamiltonian connected, which extends several former results in this area.

(4) Group coloring was first introduced by Jeager et al. [21], who introduced group coloring of graphs. Prior research on group chromatic number was restricted to simple graphs, and considered only Abelian groups in the definition of $\chi_{g}(G)$. The behavior of group coloring for multigraphs is different to that of simple graphs. Thus we extend the definition of group coloring by considering general groups (both Abelian groups and non-Abelian groups), and investigate the properties of $\chi_{g}$ for multigraphs by proving an analogue to Brooks' Theorem. 


\section{Chapter 2}

\section{Hamiltonian properties in Cartesian product}

\subsection{Introduction}

All graphs considered in this chapter are finite loopless simple graphs.

Let $G_{1}=\left(V_{1}, E_{1}\right)$ and $G_{2}=\left(V_{2}, E_{2}\right)$ be two graphs. The Cartesian product of $G_{1}$ and $G_{2}$, denoted by $G_{1} \times G_{2}$, is the graph with vertex set $V_{1} \times V_{2}$ such that two vertices $\left(x_{1}, y_{1}\right)$ and $\left(x_{2}, y_{2}\right)$ are adjacent if and only if either $x_{1}=x_{2} \in V_{1}$ with $y_{1} y_{2} \in E_{2}$, or $y_{1}=y_{2} \in V_{2}$ with $x_{1} x_{2} \in E_{1}$. It follows from the definition that for any $(x, y) \in V\left(G_{1} \times G_{2}\right)$,

$$
d_{G_{1} \times G_{2}}(x, y)=d_{G_{1}}(x)+d_{G_{2}}(y) .
$$

For any $y \in V_{2}$, define $\left(G_{1}\right)_{y}$ to be the graph with vertex set $\left(V_{1}\right)_{y}=\left\{(x, y) \mid x \in V_{1}\right\}$ and edge set $\left(E_{1}\right)_{y}=\left\{\left(x_{1}, y\right)\left(x_{2}, y\right) \mid x_{1} x_{2} \in E_{1}\right\}$. Similarly, For any $x \in V_{1}$, define $\left(G_{2}\right)_{x}$ to be the graph with vertex set $\left(V_{2}\right)_{x}=\left\{(x, y) \mid y \in V_{2}\right\}$ and edge set $\left(E_{2}\right)_{x}=$ $\left\{\left(x, y_{1}\right)\left(x, y_{2}\right) \mid y_{1} y_{2} \in E_{2}\right\}$.

Note that $\left(G_{1}\right)_{y}$ is isomorphic to graph $G_{1}$, for any $y \in V_{2}$; and that $\left(G_{2}\right)_{x}$ is isomorphic 
to graph $G_{2}$ for any $x \in V_{1}$. It is clear that

$$
\begin{gathered}
\left(V_{1}\right)_{y} \cap\left(V_{1}\right)_{y^{\prime}}=\emptyset \quad\left(E_{1}\right)_{y} \cap\left(E_{1}\right)_{y^{\prime}}=\emptyset \quad \text { for } y \neq y^{\prime} ; \\
\left(V_{2}\right)_{x} \cap\left(V_{2}\right)_{x^{\prime}}=\emptyset \quad\left(E_{2}\right)_{x} \cap\left(E_{2}\right)_{x^{\prime}}=\emptyset \quad \text { for } x \neq x^{\prime} ; \\
\left(V_{1}\right)_{y} \cap\left(V_{2}\right)_{x}=\{(x, y)\} \quad \text { for } x \in V_{1} ; \quad y \in V_{2} ; \\
E\left(G_{1} \times G_{2}\right)=\left(\cup_{y \in V_{2}}\left(E_{1}\right)_{y}\right) \cup\left(\cup_{x \in V_{1}}\left(E_{2}\right)_{x}\right) ; \\
V\left(G_{1} \times G_{2}\right)=\cup_{y \in V_{2}}\left(V_{1}\right)_{y}=\cup_{x \in V_{1}}\left(V_{2}\right)_{x} .
\end{gathered}
$$

Define a graph to be weakly edge-pancyclic if every edge $e$ lies on a circuit of length $l$ for all $l=k, k+1, \cdots,|V(G)|$, where $k$ is the length of a minimum circuit containing $e$.

Our research is motivated by the following open problem posed in [15]:

Problem 2.1.1 (Problem 6 of [15]) What natural graph properties of $G$ and $H$ are sufficient to imply that the product of $G$ and $H$ is hamiltonian?

In this chapter, we first investigate the sufficient and necessary conditions for $G \times H$ being hamiltonian or traceable when $G$ is a hamiltonian graph and $H$ is a tree. Define $\mathfrak{F}$ to be the set of such graph $H^{(n)}$ which is obtained by identifying every degree 1 vertex of $H$ with the center of a $K_{1, n}$, where $H$ is a subdivision of $K_{1,3}$. Specially, $K_{1,3}^{(n)} \in \mathfrak{F}$. Our main theorems are as follows:

Theorem 2.1.2 Let $G_{1}$ be a hamiltonian graph with order $n$ and let $T$ be a tree with maximum degree $\Delta$. Then the graph $G=G_{1} \times T$ is hamiltonian if and only if $\Delta \leq n$.

Theorem 2.1.3 Let $G_{1}$ be a hamiltonian graph with order $n$ and let $T$ be a tree with maximum degree $\Delta$. Then the graph $G=G_{1} \times T$ is traceable if and only if either $\Delta \leq n$, or $\Delta=n+1$ and $T$ contains no member of $\mathfrak{F}$ as a subgraph.

If we strengthen the condition of $G$ in $G \times H$, then the Cartesian product will have better hamiltonian properties. 
Theorem 2.1.4 Let $G$ be a Hamiltonian connected graph of order $n$ and let $T$ be a tree. Then $G \times T$ is Hamiltonian connected if and only if $\Delta(T) \leq n-1$.

Theorem 2.1.5 Let $G$ be an edge-pancyclic graph of order $n$ and let $T$ be a tree. Then $G \times T$ is weakly edge-pancyclic if and only if $\Delta(T) \leq n-1$.

Theorem 2.1.6 Let $G$ be a pan-connected graph of order $n$ and let $T$ be a tree. Then $G \times T$ is pan-connected if and only if $\Delta(T) \leq n-1$.

In Section 2.2, we will prove some lemmas. Then from Section 2.3 to Section 2.7, we will prove Theorem 2.1.2, Theorem 2.1.3, Theorem 2.1.4, Theorem 2.1.5 and Theorem 2.1.6, respectively.

\subsection{Preliminaries}

In this section, we will show some lemmas that will be used in the proofs of our main results. The following two theorems are well known as "toughness conditions".

Theorem 2.2.1 ([4]) Let $S$ be a set of vertices of a hamiltonian graph $G$. Then $c(G-$ $S) \leq|S|$.

Theorem 2.2.2 ([4]) Let $S$ be a set of vertices of a graph $G$. If $G$ is traceable, then $c(G-S) \leq|S|+1$.

Lemma 2.2.3 Let $K_{1, m}$ be a star $(m \geq 0)$ and let $C_{n}$ be a circuit $(n \geq 3)$. If $m \leq n$, then the graph $C_{n} \times K_{1, m}$ is Hamiltonian.

Proof. We denote $G=C_{n} \times K_{1, m}$. If $m=0$, then the result holds trivially since $G$ is isomorphic to $C_{n}$. So we will assume that $m \geq 1$ in the following proof. Assume that 
$V\left(C_{n}\right)=\left\{x_{1}, x_{2}, \cdots, x_{n}\right\}$ and $V\left(K_{1, m}\right)=\left\{y_{0}, y_{1}, \cdots, y_{m}\right\}$, where $d\left(y_{0}\right)=m$ and $d\left(y_{i}\right)=1$ for $1 \leq i \leq m$. Note that $\left(G_{1}\right)_{y_{i}} \cong C_{n}$ and that $\left(G_{1}\right)_{y_{i}}=\left(x_{1}, y_{i}\right)\left(x_{2}, y_{i}\right) \cdots\left(x_{n}, y_{i}\right)\left(x_{1}, y_{i}\right)$ for $i=0,1, \cdots, m$. Then

$$
\begin{aligned}
C= & \left(\left(E_{1}\right)_{y_{1}}-\left\{\left(x_{1}, y_{1}\right)\left(x_{2}, y_{1}\right)\right\}\right) \cup \cdots \cup\left(\left(E_{1}\right)_{y_{i}}-\left\{\left(x_{i}, y_{i}\right)\left(x_{i+1}, y_{i}\right)\right\}\right) \cup \cdots \\
\cup & \left(\left(E_{1}\right)_{y_{m}}-\left\{\left(x_{m}, y_{m}\right)\left(x_{m+1}, y_{m}\right)\right\}\right) \\
\cup & \left(\left(E_{1}\right)_{y_{0}}-\left\{\left(x_{1}, y_{0}\right)\left(x_{2}, y_{0}\right),\left(x_{2}, y_{0}\right)\left(x_{3}, y_{0}\right), \cdots,\left(x_{m}, y_{0}\right)\left(x_{m+1}, y_{0}\right)\right\}\right) \\
\cup & \left\{\left(x_{1}, y_{0}\right)\left(x_{1}, y_{1}\right),\left(x_{2}, y_{0}\right)\left(x_{2}, y_{1}\right), \cdots,\left(x_{i}, y_{0}\right)\left(x_{i}, y_{i}\right),\left(x_{i+1}, y_{0}\right)\left(x_{i+1}, y_{i}\right), \cdots\right. \\
& \left.\left(x_{m}, y_{0}\right)\left(x_{m}, y_{m}\right),\left(x_{m+1}, y_{0}\right)\left(x_{m+1}, y_{m}\right)\right\}
\end{aligned}
$$

is a Hamiltonian circuit of $G$, where all indexes are taken modulo $n$.

Note 1 By the proof of Lemma $1,\left|\left(E_{1}\right)_{y_{0}} \cap E(C)\right|=\emptyset$ if $n=m$. If $n>m$, then $\left(x_{i}, y_{0}\right)\left(x_{i+1}, y_{0}\right) \in E(C)$ for $m+1 \leq i \leq n$, that is, $\left|\left(E_{1}\right)_{y_{0}} \cap E(C)\right|=n-m$. On the other hand, we can construct another Hamiltonian circuit $C^{\prime}$ such that $\left(x_{i}, y_{0}\right)\left(x_{i+1}, y_{0}\right) \in E\left(C^{\prime}\right)$ for $m+j \leq i \leq n+j$ by $\left(G_{1}\right)_{y_{i}} \cong C_{n}$ for $i=0,1, \cdots, m$, where $1 \leq j \leq n$ and all indexes are taken modulo $n$.

Lemma 2.2.4 Let $C_{n}$ be a circuit of $n$ vertices $(n \geq 3)$ and let $K_{1, n+1}$ be a star with center $y_{0}$. Then in the graph $C_{n} \times K_{1, n+1}$, for any pair of adjacent vertices $u_{1}, u_{2} \in V\left(C_{n}\right)$ and any pair of distinct degree one vertices $v_{1}, v_{2} \in V\left(K_{1, n+1}\right)$, there is a Hamiltonian path $P$ of $C_{n} \times K_{1, n+1}$ satisfying:

(i) $\left(u_{1}, v_{1}\right)$ and $\left(u_{2}, v_{2}\right)$ are endpoints of $P$;

(ii) For $i=1$ or $2, P \backslash\left(G_{1}\right)_{v_{i}}$ is a Hamiltonian path of $C_{n} \times\left(K_{1, n+1}-v_{i}\right)$ with endpoints $\left(u_{3-i}, v_{3-i}\right)$ and $\left(u_{3-i}, y_{0}\right)$.

Proof. Let $\left\{u_{1}, u_{2}, v_{1}, v_{2}\right\}$ be given vertices such that $u_{1} u_{2} \in E\left(C_{n}\right)$ and $v_{1}, v_{2} \in$ $D_{1}\left(K_{1, n+1}\right)$. Let $V\left(C_{n}\right)=\left\{x_{1}, x_{2}, \cdots, x_{n}\right\}$ and $V\left(K_{1, n+1}\right)=\left\{y_{0}, y_{1}, \cdots, y_{n+1}\right\}$, where $d\left(y_{0}\right)=n+1$ and $d\left(y_{i}\right)=1$ for $1 \leq i \leq n+1$. By the symmetry of $C_{n}$ and $K_{1, n+1}$, we 
can assume that $u_{1}=x_{1}, u_{2}=x_{2}, v_{1}=y_{1}$ and $v_{2}=y_{n+1}$, . Then

$$
\begin{aligned}
P= & \left(\left(E_{1}\right)_{y_{1}}-\left\{\left(x_{1}, y_{1}\right)\left(x_{2}, y_{1}\right)\right\}\right) \cup \cdots \cup\left(\left(E_{1}\right)_{y_{i}}-\left\{\left(x_{i}, y_{i}\right)\left(x_{i+1}, y_{i}\right)\right\}\right) \cup \cdots \\
\cup & \left(\left(E_{1}\right)_{y_{n+1}}-\left\{\left(x_{1}, y_{n+1}\right)\left(x_{2}, y_{n+1}\right)\right\}\right) \\
\cup & \left\{\left(x_{2}, y_{0}\right)\left(x_{2}, y_{1}\right),\left(x_{2}, y_{0}\right)\left(x_{2}, y_{2}\right), \cdots,\left(x_{i}, y_{0}\right)\left(x_{i}, y_{i-1}\right),\left(x_{i}, y_{0}\right)\left(x_{i}, y_{i}\right), \cdots\right. \\
& \left.\left(x_{n}, y_{0}\right)\left(x_{n}, y_{n-1}\right),\left(x_{n}, y_{0}\right)\left(x_{n}, y_{n}\right),\left(x_{1}, y_{0}\right)\left(x_{1}, y_{n}\right),\left(x_{1}, y_{0}\right)\left(x_{1}, y_{n+1}\right),\right\}
\end{aligned}
$$

is a required Hamiltonian path of $C_{n} \times K_{1, n+1}$.

Let $K_{1,3}^{(n)}$ be the graph obtained by identifying every degree 1 vertex of a $K_{1,3}$ with the center of a $K_{1, n}$. Note that $\Delta\left(K_{1,3}^{(n)}\right)=n+1$.

Lemma 2.2.5 Let $C_{n}$ be a circuit $(n \geq 3)$. Then $C_{n} \times K_{1,3}^{(n)}$ is not traceable.

Proof. We denote $G=C_{n} \times K_{1,3}^{(n)}$. By way of contradiction, we may assume that $P$ is a Hamiltonian path of $G$. Referring to the structure of $K_{1,3}^{(n)}$, we may assume that $T_{1}$, $T_{2}$ and $T_{3}$ are the three copies of $K_{1, n}$ whose centers were identified with the degree one vertices of $K_{1,3}$. Let $y_{i}$ be the center of $T_{i}(i=1,2,3)$. Note that the $T_{i}$ can be also viewed as a sub-tree of $K_{1,3}^{(n)}$, and then $C_{n} \times T_{i}$ can be viewed as a subgraph of $G(i=1,2,3)$.

Suppose $C_{n} \times T_{1}$ contains no endpoints of $P$. Assume that $\left\{z_{1}, z_{2}, \ldots, z_{n}\right\}$ are the degree one vertices in $T_{1}$. Then $\left(G_{1}\right)_{z_{1}},\left(G_{1}\right)_{z_{2}}, \ldots,\left(G_{1}\right)_{z_{n}}$ and $C_{n} \times\left(K_{1,3}^{(n)}-T_{1}\right)$ are the $n+1$ components of $G-\left(G_{1}\right)_{y_{1}}$. Since $P$ is a spanning subgraph of $G, c\left(P-\left(G_{1}\right)_{y_{1}}\right) \geq$ $c\left(G-\left(G_{1}\right)_{y_{1}}\right)=n+1$. By Theorem 2.2.2, $\left.c\left(P-\left(G_{1}\right)_{y_{1}}\right) \leq \mid\left(V_{1}\right)_{y_{1}}\right) \mid+1=n+1$. Thus, $c\left(P-\left(G_{1}\right)_{y_{1}}\right)=n+1$. Note that every $\left(G_{1}\right)_{z_{i}}(1 \leq i \leq n)$ contains at least one component of $P-\left(G_{1}\right)_{y_{1}}$, and $C_{n} \times\left(K_{1,3}^{(n)}-T_{1}\right)$ contains at least two components of $P-\left(G_{1}\right)_{y_{1}}$ as it contains both endpoints of $P$. Therefore, $c\left(P-\left(G_{1}\right)_{y_{1}}\right) \geq n+2$, which contradicts to $c\left(P-\left(G_{1}\right)_{y_{1}}\right)=n+1$. So $C_{n} \times T_{1}$ contains at least one endpoint of $P$. Similarly, each of $C_{n} \times T_{2}$ and $C_{n} \times T_{3}$ contains at least one endpoint of $P$. Since $C_{n} \times T_{1}, C_{n} \times T_{2}$ and $C_{n} \times T_{3}$ are disjoint subgraphs of $G, P$ has at least three endpoints, a contradiction.

Corollary 2.2.6 Let $C_{n}$ be a circuit, where $n \geq 3$. For any tree $T$, if $T$ contains a member of $\mathfrak{F}$ as a subgraph, then $G=C_{n} \times T$ is not traceable. 
The proof of this corollary is similar to the proof of Lemma 2.2.5. So it is omitted.

Let $P_{m}=p_{1} p_{2} \cdots p_{m}$ be a path with $m$ vertices. Denote $P_{m}^{(n)}$ to be the graph obtained by identifying $p_{i}$ with the center of a star $T_{i}$ where $T_{i}=K_{1, n-1}$ for $i=1$ or $m$ and $T_{i}=K_{1, n-2}$ for $2 \leq i \leq m-1$ if $m \geq 2$ and $P_{m}^{(n)}=K_{1, n}$ if $m=1$. Thus every vertex of $P_{m}^{(n)}$ is either of degree one or of degree $n$. Note that $P_{m}$ and $T_{i}(1 \leq i \leq m)$ can be viewed as subgraphs of $P_{m}^{(n)}$.

Let $C_{n}$ be a circuit $(n \geq 3)$. Let $u_{1}$ and $u_{2}$ be adjacent vertices of $C_{n}$, and let $v_{1}$ and $v_{2}$ be degree one vertices of $P_{m}^{(n+1)}$ where $v_{1} \in T_{1}$ and $v_{2} \in T_{m}$. In $C_{n} \times P_{m}^{(n+1)}$, a path $P$ is called a Hamiltonian path with respect to $\left\{u_{1}, u_{2}, v_{1}, v_{2}\right\}$ if $P$ satisfies:

(i) $P$ is a Hamiltonian path where $\left(u_{2}, v_{2}\right)$ is an endpoint and the other endpoint is in $\left(C_{n}\right)_{v_{1}}$;

(ii) $P-\left(C_{n}\right)_{v_{1}}$ is a Hamiltonian path of $C_{n} \times\left(P_{m}^{(n+1)}-v_{1}\right)$ with an endpoint in $\left(C_{n}\right)_{p_{1}}$;

(ii) $P-\left(C_{n}\right)_{v_{2}}$ is a Hamiltonian path of $C_{n} \times\left(P_{m}^{(n+1)}-v_{2}\right)$ with an endpoint $\left(u_{1}, p_{m}\right)$.

Lemma 2.2.7 Let $C_{n}$ be a circuit $(n \geq 3)$ and $G=C_{n} \times P_{m}^{(n+1)}$. Then $G$ is traceable. Moreover, for any two adjacent vertices $u_{1}, u_{2} \in V\left(C_{n}\right)$ and any two degree one vertices $v_{1}, v_{2}$ of $P_{m}^{(n+1)}$ where $v_{1} \in T_{1}$ and $v_{2} \in T_{m}$, there is a Hamiltonian path $P$ of $G$ with respect to $\left\{u_{1}, u_{2}, v_{1}, v_{2}\right\}$.

Proof. We argue by induction on $m$. If $m=1$, then the result follows by Lemma 2.2.4. So assume $m \geq 2$ and the result holds for smaller values of $m$. Let $\left\{u_{1}, u_{2}, v_{1}, v_{2}\right\}$ be given vertices satisfying the hypotheses.

As $T_{m} \subseteq P_{m}^{(n+1)}$, denote $H_{1}=P_{m}^{(n+1)} / T_{m}$ and $H_{2}=P_{m}^{(n+1)} /\left(P_{m}^{(n+1)}-T_{m}\right)$. Then $H_{1} \cong P_{m-1}^{(n+1)}$ and $H_{2} \cong P_{1}^{(n+1)}=K_{1, n+1}$. Assume that $v_{0} \in V\left(H_{1}\right)$ is the vertex to which $T_{m}$ contracts and $v_{0}^{\prime} \in V\left(H_{2}\right)$ is the vertex to which $P_{m}^{(n+1)}-T_{m}$ contracts.

By induction hypotheses, $C_{n} \times H_{1}$ has a Hamiltonian path $P_{1}$ with respect to $\left\{u_{2}, u_{1}, v_{1}, v_{0}\right\}$. Note that $\left(u_{2}, p_{m-1}\right)$ is an endpoint of $P_{1}-\left(C_{n}\right)_{v_{0}}$. By Lemma 2.2.4, $C_{n} \times H_{2}$ has a Hamiltonian path $P_{2}$ with endpoint $\left(u_{1}, v_{0}^{\prime}\right)$ and $\left(u_{2}, v_{2}\right)$ such that $\left(u_{2}, p_{m}\right)$ is an endpoint 
of $P_{2}-\left(C_{n}\right)_{v_{0}^{\prime}}$ and $\left(u_{1}, p_{m}\right)$ is an endpoint of $P_{2}-\left(C_{n}\right)_{v_{2}}$. Therefore

$$
P=\left(P_{1}-\left(C_{n}\right)_{v_{0}}\right) \cup\left(P_{2}-\left(C_{n}\right)_{v_{0}^{\prime}}\right) \cup\left\{\left(u_{2}, p_{m-1}\right)\left(u_{2}, p_{m}\right)\right\}
$$

is a Hamiltonian path of $G$ with respect to $\left\{u_{1}, u_{2}, v_{1}, v_{2}\right\}$.

Corollary 2.2.8 Let $C_{n}$ be a circuit $(n \geq 3)$ and let $T$ be a tree obtained by subdividing some edges of $P_{m}$ in $P_{m}^{(n+1)}(m \geq 2)$. Then $C_{n} \times T$ is traceable. Moreover, for any two adjacent vertices $u_{1}, u_{2} \in V\left(C_{n}\right)$ and any two degree one vertices $v_{1}$, $v_{2}$ of $P_{m}^{(n+1)}$ where $v_{1} \in T_{1}$ and $v_{2} \in T_{m}, C_{n} \times T$ admits a Hamiltonian path $P$ with respect to $\left\{u_{1}, u_{2}, v_{1}, v_{2}\right\}$.

Proof. Let $\left\{u_{1}, u_{2}, v_{1}, v_{2}\right\}$ be given vertices satisfying the hypotheses. It's sufficient to show that the result holds when $T$ is obtained by subdividing one edge of $P_{m}$. Assume that $T$ is obtained by subdividing $p_{k} p_{k+1} \in E\left(P_{m}\right)(k \geq 1)$ and $v_{0}$ is the subdividing vertex.

Let $C_{1}$ and $C_{2}$ be the two components of $T-v_{0}$ where $p_{k} \in V\left(C_{1}\right)$. Denote $H_{i}$ to be the induced subgraph of $V\left(C_{i}\right) \cup v_{0}$ in $T(i=1,2)$. Then $V\left(H_{1}\right) \cap V\left(H_{2}\right)=v_{0}$, and $H_{1} \cong P_{k}^{n+1}, H_{2} \cong P_{m-k-1}^{n+1}$. Therefore, by Lemma 2.2.5, $C_{n} \times H_{2}$ has a Hamiltonian path $P_{2}$ with respect to $\left\{u_{1}, u_{2}, v_{0}, v_{2}\right\}$. Assume that $\left(x_{j}, p_{k+1}\right)$ is the endpoint of $P_{2}-\left(C_{n}\right)_{v_{0}}$ in $\left(C_{n}\right)_{p_{k+1}}\left(x_{j} \in V\left(C_{n}\right)\right)$. Let $x_{j+1}$ be a neighbor of $x_{j}$ in $C_{n}$. By Lemma 2.2.5, $C_{n} \times H_{1}$ has a Hamiltonian path $P_{1}$ with respect to $\left\{x_{j+1}, x_{j}, v_{1}, v_{0}\right\}$. Hence,

$$
\begin{aligned}
P= & \left(P_{1}-\left(C_{n}\right)_{v_{0}}\right) \cup\left(P_{2}-\left(C_{n}\right)_{v_{0}}\right) \cup\left(E\left(\left(C_{n}\right)_{v_{0}}\right)-\left(x_{j}, v_{0}\right)\left(x_{j+1}, v_{0}\right)\right) \\
& \cup\left\{\left(x_{j+1}, p_{k}\right)\left(x_{j+1}, v_{0}\right),\left(x_{j}, v_{0}\right)\left(x_{j}, p_{k+1}\right)\right\}
\end{aligned}
$$

is a Hamiltonian path of $C_{n} \times T$ with respect to $\left\{u_{1}, u_{2}, v_{1}, v_{2}\right\}$.

Lemma 2.2.9 Let $C_{n}$ be a circuit (where $n \geq 3$ ). Let $T$ be a tree where $y_{1} y_{2} \in E(T)$ and $y_{1} \in D_{1}(T)$. If $d_{T}\left(y_{2}\right) \leq n$ and $C_{n} \times\left(T-y_{1}\right)$ is traceable, then $C_{n} \times T$ is traceable.

Proof. Let $P$ be a Hamiltonian path of $C_{n} \times\left(T-y_{1}\right)$. Since $d_{T-y_{1}}\left(y_{2}\right) \leq n-1$, at least one edge of $\left(C_{n}\right)_{y_{2}}$ lies in $P$. Without loss of generality, we can assume that 
$\left(x_{1}, y_{2}\right)\left(x_{2}, y_{2}\right) \in E\left(\left(C_{n}\right)_{y_{2}}\right) \cap E(P)$ where $x_{1} x_{2} \in E\left(C_{n}\right)$. Hence

$$
\begin{aligned}
P^{\prime} & =\left(P-\left\{\left(x_{1}, y_{2}\right)\left(x_{2}, y_{2}\right)\right\}\right) \cup\left(E\left(\left(C_{n}\right)_{y_{1}}\right)-\left\{\left(x_{1}, y_{1}\right)\left(x_{2}, y_{1}\right)\right\}\right) \\
& \cup\left\{\left(x_{1}, y_{1}\right)\left(x_{1}, y_{2}\right),\left(x_{2}, y_{1}\right)\left(x_{2}, y_{2}\right)\right\}
\end{aligned}
$$

is a Hamiltonian path of $C_{n} \times T$.

Lemma 2.2.10 Let $G$ be a Hamiltonian connected graph of order $n$. Then $G \times K_{1, n}$ is not Hamiltonian connected.

Proof. Let $G_{0}=G \times K_{1, n}$. Suppose $G_{0}$ is Hamiltonian connected. Let $y$ be the center of $K_{1, n}$. Choose $\left(x_{1}, y\right),\left(x_{2}, y\right) \in V\left(G_{0}\right)$. Then there is an $\left(\left(x_{1}, y\right),\left(x_{2}, y\right)\right)$-Hamiltonian path $P$ of $G_{0}$. Since $c\left(G_{0}-V\left((G)_{y}\right)\right)=n$ and $P-V\left((G)_{y}\right)$ is a spanning subgraph of $G_{0}-V\left((G)_{y}\right), c\left(P-V\left((G)_{y}\right)\right) \geq n$. Note that $P-\left\{\left(x_{1}, y\right),\left(x_{2}, y\right)\right\}$ is still a path. Thus by Theorem 2.2.2,

$$
\begin{aligned}
c\left(P-V\left((G)_{y}\right)\right) & =c\left(\left(P-\left\{\left(x_{1}, y\right),\left(x_{2}, y\right)\right\}\right)-\left(V\left((G)_{y}\right) \backslash\left\{\left(x_{1}, y\right),\left(x_{2}, y\right)\right\}\right)\right) \\
& \leq\left|\left(V\left((G)_{y}\right) \backslash\left\{\left(x_{1}, y\right),\left(x_{2}, y\right)\right\}\right)\right|+1 \\
& =n-1
\end{aligned}
$$

which contradicts to $c\left(P-V\left((G)_{y}\right)\right) \geq n$.

Lemma 2.2.11 Let $G$ be a Hamiltonian connected graph of order $n$. Let $H$ be a path of length one. Then $G \times H$ is Hamiltonian connected.

Proof. We may assume that $H=z_{1} z_{2}$. Let $\left(x_{1}, y_{1}\right)$ and $\left(x_{2}, y_{2}\right)$ be a pair of distinct vertices in $G \times H$. Without loss of generality, we may assume that $y_{1}=z_{1}$.

If $y_{1} \neq y_{2}$, then $y_{2}=z_{2}$. Choose $x_{3} \in V(G) \backslash\left\{x_{1}, x_{2}\right\}$. Since $(G)_{z_{i}} \cong G$ is Hamiltonian connected, there is a Hamiltonian $\left(\left(x_{i}, z_{i}\right),\left(x_{3}, z_{i}\right)\right)$-path $P_{i}$ of $(G)_{z_{i}}(i=1,2)$. Then $P_{1}+P_{2}+\left(x_{3}, z_{1}\right)\left(x_{3}, z_{2}\right)$ is a Hamiltonian $\left(\left(x_{1}, y_{1}\right),\left(x_{2}, y_{2}\right)\right)$-path of $G \times H$.

So we could assume that $y_{1}=y_{2}=z_{1}$. Then $x_{1} \neq x_{2}$. Since $(G)_{z_{1}} \cong G$ is Hamiltonian connected, there is a $\left(\left(x_{1}, z_{1}\right),\left(x_{2}, z_{1}\right)\right)$-Hamiltonian path $P_{1}$ of $(G)_{z_{1}}$. Choose 
$\left(x_{3}, z_{1}\right)\left(x_{4}, z_{1}\right) \in E\left(P_{1}\right)$. Since $(G)_{z_{2}} \cong G$ is Hamiltonian connected, there exists a $\left(\left(x_{3}, z_{2}\right),\left(x_{4}, z_{2}\right)\right)$-Hamiltonian path $P_{2}$ of $(G)_{z_{2}}$. Thus

$$
P=\left(P_{1}-\left(x_{3}, z_{1}\right)\left(x_{4}, z_{1}\right)\right)+P_{2}+\left\{\left(x_{3}, z_{1}\right)\left(x_{3}, z_{2}\right),\left(x_{4}, z_{1}\right)\left(x_{3}, z_{2}\right)\right\}
$$

is a Hamiltonian $\left(\left(x_{1}, y_{1}\right),\left(x_{2}, y_{2}\right)\right)$-path of $G \times H$.

Lemma 2.2.12 Let $G$ be an edge-pancyclic graph of order $n$. Then $G \times K_{1, n}$ is not weakly edge-pancyclic.

Proof. Let $G_{0}=G \times K_{1, n}$. Suppose $G_{0}$ is weakly edge-pancyclic. Let $y$ be the center of $K_{1, n}$. Choose $x_{1} x_{2} \in E(G)$. Then by the definition of weakly edge-pancyclic graph, there is a Hamiltonian circuit $C$ of $G_{0}$ containing $\left(x_{1}, y\right)\left(x_{2}, y\right)$. Since $c\left(G_{0}-V\left((G)_{y}\right)\right)=n$, $c\left(C-V\left((G)_{y}\right)\right) \geq n$. Note that $C-\left\{\left(x_{1}, y\right),\left(x_{2}, y\right)\right\}$ is a path. Thus by Theorem 2.2.2,

$$
\begin{aligned}
c\left(C-V\left((G)_{y}\right)\right) & =c\left(\left(C-\left\{\left(x_{1}, y\right),\left(x_{2}, y\right)\right\}\right)-\left(V\left((G)_{y}\right) \backslash\left\{\left(x_{1}, y\right),\left(x_{2}, y\right)\right\}\right)\right) \\
& \leq\left|V\left((G)_{y}\right) \backslash\left\{\left(x_{1}, y\right),\left(x_{2}, y\right)\right\}\right|+1 \\
& =n-1,
\end{aligned}
$$

which contradicts to $c\left(C-V\left(G_{y}\right)\right) \geq n$.

Lemma 2.2.13 Let $G$ be an edge-pancyclic graph of order $n$ and let $H=y_{1} y_{2}$ be a path of length 1 . Then for $\forall x \in V(G),\left(x, y_{1}\right)\left(x, y_{2}\right)$ is on a circuit of length $l$ in $G \times H$ for all $l=4,5, \cdots, 2 n$.

Proof. Fix $x \in V(G)$. We will show that for all $l=4,5, \cdots, 2 n$, there is a circuit $C_{l}$ of length $l$ containing $\left(x, y_{1}\right)\left(x, y_{2}\right)$.

Since $G$ is edge-pancyclic, there is a triangle $x z_{1} z_{2}$ in $G$, where $z_{1}, z_{2} \in N_{G}(x)$. Then $C_{4}=\left(x, y_{1}\right)\left(x, y_{2}\right)\left(z_{1}, y_{2}\right)\left(z_{1}, y_{1}\right)$ and $C_{5}=\left(x, y_{1}\right)\left(x, y_{2}\right)\left(z_{1}, y_{2}\right)\left(z_{1}, y_{1}\right)\left(z_{2}, y_{1}\right)$. So we may assume that $l \geq 6$. Let $l_{1}=\lfloor l / 2\rfloor$ and $l_{2}=l-l_{1}$. Note that both $l_{1}$ and $l_{2}$ are at least 3. Since $(G)_{y_{i}} \cong G$ is edge-pancyclic, there is a circuit $C_{y_{i}}^{l_{i}}$ of length $l_{i}$ containing $\left(x, y_{i}\right)\left(z_{1}, y_{i}\right)$ in $(G)_{y_{i}}(i=1,2)$. Thus $C_{l}=\left(C_{y_{1}}^{l_{1}}-\left(x, y_{1}\right)\left(z_{1}, y_{1}\right)\right)+\left(C_{y_{2}}^{l_{2}}-\left(x, y_{2}\right)\left(z_{1}, y_{2}\right)\right)+$ $\left\{\left(x, y_{1}\right)\left(x, y_{2}\right),\left(z_{1}, y_{1}\right)\left(z_{1}, y_{2}\right)\right\}$. 
Lemma 2.2.14 Let $n$ and $k$ be two positive integers such that $n \geq 3$ and $k \leq n-1$. Let $\left\{n_{1}, n_{2}, \cdots, n_{k}\right\}$ be a sequence of positive integers. Then for each integer $l \in[n+2, n+$ $\left.\sum_{i=1}^{k}\left(n_{i} * n\right)\right]$, there is a sequence of integers $\left\{l_{1}, l_{2}, \cdots, l_{k}\right\}$ such that $l=n+\sum_{i=1}^{k} l_{i}$, and $l_{i}$ is either 0 or in the interval $\left[2, n_{i} * n\right]$ for all $i=1,2, \cdots, k$.

Proof. If $n+2 \leq l \leq n+n_{1} * n$, then $\left\{l_{1}, l_{2}, \cdots, l_{k}\right\}=\{l-n, 0, \cdots, 0\}$. So we assume that $l \geq n+n_{1} * n+1$. If $l=n+\sum_{i=1}^{j}\left(n_{i} * n\right)+1(1 \leq j \leq k-1)$, then $\left\{l_{1}, l_{2}, \cdots, l_{k}\right\}=$ $\left\{n_{1} * n-1, n_{2} * n, \cdots, n_{j} * n, 2,0, \cdots, 0\right\}$. If $l=n+\sum_{i=1}^{j}\left(n_{i} * n\right)+l^{\prime}(1 \leq j \leq k-1$ and $\left.2 \leq l^{\prime} \leq n_{j+1} * n\right)$, then $\left\{l_{1}, l_{2}, \cdots, l_{k}\right\}=\left\{n_{1} * n, n_{2} * n, \cdots, n_{j} * n, l^{\prime}, 0, \cdots, 0\right\}$.

\subsection{Hamiltonian circuit in $G \times T$}

In this section, we will prove Theorem 2.1.2. We first prove the following theorem, and Theorem 2.1.2 will be its corollary.

Theorem 2.3.1 Let $T$ be a tree with maximum degree $\Delta$. Then the graph $G=C_{n} \times T$ is Hamiltonian if and only if $\Delta \leq n$.

Proof of Theorem 2.3.1 Let $G=C_{n} \times T$ be a graph with a Hamiltonian circuit. If there exists $y \in V(T)$ such that $d_{T}(y) \geq n+1$, then $c\left(G-\left(C_{n}\right)_{y}\right)=c(T-y)=d_{T}(y) \geq n+1$. However, by Theorem 2.2.1, $c\left(G-\left(C_{n}\right)_{y}\right) \leq\left|V\left(\left(C_{n}\right)_{y}\right)\right|=n$, a contradiction. Therefore $d_{T}(y) \leq n$ for every $y \in V(T)$ and $\Delta \leq n$.

So it is sufficient to show that if $\Delta \leq n$, then $G=C_{n} \times T$ has a Hamiltonian circuit. If $T$ is a star, then it follows Lemma 2.2.3. Therefore we may assume that $T$ is not a star. By way of contradiction, choose a tree $T$ with minimal number of vertices such that $\Delta \leq n$ and $G=C_{n} \times T$ has no Hamiltonian circuit.

Note that $T$ can be viewed as a graph obtained from finite stars $T_{1}, T_{2}, \cdots, T_{k}$ by connecting their centers with edges and there exists such a star $T_{i}$ that is connected to the other stars with only one edge. Without loss of generality, we may assume that $T_{1}$ 
is only connected to $T_{2}$. Let $y_{i}$ be the center of $T_{i}(i=1,2)$. Since $T-T_{1}$ is also a tree and $\left|V\left(T-T_{1}\right)\right|<|V(T)|, C_{n} \times\left(T-T_{1}\right)$ has a Hamiltonian circuit $C$. Since $d_{T-T_{1}}\left(y_{2}\right) \leq n-1$, at least one edge of $\left(C_{n}\right)_{y_{2}}$ lies in $C$. Without loss of generality, we can assume that $\left(x_{1}, y_{2}\right)\left(x_{2}, y_{2}\right) \in E\left(\left(C_{n}\right)_{y_{2}}\right) \cap E(C)$ where $x_{1} x_{2} \in E\left(C_{n}\right)$. Since $\Delta\left(T_{1}\right) \leq n-1$, by Lemma 2.2.3, there is a Hamiltonian circuit $C^{\prime}$ of $C_{n} \times T_{1}$. By Note 1 , we can assume that $\left(x_{1}, y_{1}\right)\left(x_{2}, y_{1}\right) \in E\left(C^{\prime}\right)$. Hence

$$
\begin{aligned}
C^{\prime \prime} & =\left(C-\left\{\left(x_{1}, y_{2}\right)\left(x_{2}, y_{2}\right)\right\}\right) \cup\left(C^{\prime}-\left\{\left(x_{1}, y_{1}\right)\left(x_{2}, y_{1}\right)\right\}\right) \\
& \cup\left\{\left(x_{1}, y_{1}\right)\left(x_{1}, y_{2}\right),\left(x_{2}, y_{1}\right)\left(x_{2}, y_{2}\right)\right\}
\end{aligned}
$$

is a Hamiltonian circuit of $G$, a contradiction to the choice of $T$. This completes the proof of Theorem 2.3.1.

Theorem 2.1.2 will be a corollary of Theorem 2.3.1.

\subsection{Hamiltonian path in $G \times T$}

Recall that $\mathfrak{F}$ is be the set of such graph $H^{(n)}$ which is obtained by identifying every degree 1 vertex of $H$ with the center of a $K_{1, n}$, where $H$ is a subdivision of $K_{1,3}$. Specially, $K_{1,3}^{(n)} \in \mathfrak{F}$. In this section, we will prove Theorem 2.1.3. We first prove the following theorem, and Theorem 2.1.2 will be its corollary.

Theorem 2.4.1 Let $T$ be a tree with maximum degree $\Delta$. Then the graph $G=C_{n} \times T$ is traceable if and only if $\Delta \leq n$ or $\Delta=n+1$ and $T$ contains no member of $\mathfrak{F}$ as a subgraph.

Proof of Theorem 2.4.1 By Theorem 2.3.1, we only need to consider $T$ with $\Delta \geq n+1$.

Let $G=C_{n} \times T$ be a graph with a Hamiltonian path $P$ and $\Delta \geq n+1$. If $\Delta \geq n+2$, choose $y \in V(T)$ such that $d_{T}(y) \geq n+2$. Then $c\left(G-\left(C_{n}\right)_{y}\right)=c(T-y)=d_{T}(y) \geq n+2$. However, by Theorem 2.1.2, $c\left(G-\left(C_{n}\right)_{y}\right) \leq\left|V_{1 y}\right|+1=n+1$, a contradiction. Therefore $\Delta=n+1$. By Corollary 2.2.6, $T$ contains no member of $\mathfrak{F}$ as a subgraph. 
So it is sufficient to show that if $\Delta=n+1$ and $T$ contains no member of $\mathfrak{F}$ as a subgraph, then $G=C_{n} \times T$ has a Hamiltonian path. If $T$ is a star, then it follows Lemma 2.2.4. So we may assume that $T$ is not a star. By way of contradiction, let $T$ be a tree with minimum vertices such that $T$ contains no member of $\mathfrak{F}$ as a subgraph and $G=C_{n} \times T$ is not traceable.

Claim $1\left|D_{n+1}(T)\right| \geq 2$.

Suppose $\left|D_{n+1}(T)\right|=|\{y\}|=1$. Let $y_{1}$ be a vertex of $T$ such that the distance between $y$ and $y_{1}$ in $T$ is maximum. Then $y_{1} \in D_{1}(T)$. Assume that $y_{2}$ is the only neighbor of $y_{1}$ in $T$. Since $T$ is not a star, $y_{2} \neq y$, and then $d_{T}\left(y_{2}\right) \leq n$. Since $T-y_{1}$ is also a tree and $\left|V\left(T-y_{1}\right)\right|<|V(T)|, C_{n} \times\left(T-y_{1}\right)$ has a Hamiltonian path $P$. By Lemma 2.2.9, $C_{n} \times T$ is traceable, a contradiction. This completes the proof of Claim 1 .

Choose $z_{1}, z_{2} \in D_{n+1}(T)$ such that the distance between $z_{1}$ and $z_{2}$ in $T$ is maximal. Let $P_{0}$ be the path between $z_{1}$ and $z_{2}$ in $T$.

Claim $2 D_{n+1}(T) \subseteq V\left(P_{0}\right)$

If $D_{n+1}(T)-V\left(P_{0}\right) \neq \emptyset$, then $T$ contains no member of $\mathfrak{F}$ as a subgraph, a contradiction. This completes the proof of Claim 2.

Claim 3 For every $y \in D_{1}(T)$, the distance from $y$ to $P_{0}$ is one.

By way of contradiction, assume that there exists a vertex $y_{1} \in D_{1}(T)$ such that the distance from $v$ to $P$ is greater than 1 . Assume that $y_{2}$ is the only neighbor of $y_{1}$ in $T$. Note that $y_{2} \notin V(P)$, and then $d_{T}\left(y_{2}\right) \leq n$. Since $T-y_{1}$ is also a tree and $\left|V\left(T-y_{1}\right)\right|<|V(T)|, C_{n} \times\left(T-y_{1}\right)$ has a Hamiltonian path P. By Lemma 2.2.9, $C_{n} \times T$ 
is traceable, a contradiction. This completes the proof of Claim 3.

Claim $4 V(T)-V(P)=D_{1}(T)$.

Claim 4 follows directly from Claim 3.

Claim $5 V\left(P_{0}\right)=D_{2}(T) \cup D_{n+1}(T)$.

Suppose there exists $y_{2} \in V\left(P_{0}\right) \backslash\left(D_{2}(T) \cup D_{n+1}(T)\right)$. Then there exists $y_{1} \in N_{T}\left(y_{2}\right)$ such that $y_{1} \notin V\left(P_{0}\right)$. By Claim $4, y_{1} \in D_{1}(T)$. Since $T-y_{1}$ is also a tree and $\left|V\left(T-y_{1}\right)\right|<|V(T)|, C_{n} \times\left(T-y_{1}\right)$ has a Hamiltonian path P. By Lemma 2.2.9, $C_{n} \times T$ is traceable, a contradiction. This completes the proof of Claim 5 .

By Claim 4 and 5, $T$ is either $P_{m}^{n+1}$ or a subdivision of $P_{m}^{n+1}$ by subdividing some edges of $P_{m}(m \geq 2)$. By Lemma 2.2.7 and Corollary 2.2.8, $G=C_{n} \times T$ is traceable, a contradiction. This completes the proof of Theroem 2.4.1.

Theorem 2.1.2 will be a corollary of Theorem 2.3.1.

\subsection{Hamiltonian connected Cartesian product}

In this section, we will prove Theorem 2.1.4.

Proof of Theorem 2.1.4 Let $G$ be a Hamiltonian connected graph of order $n$ and let $T$ be a tree. The necessary condition follows by Lemma 2.2.10. So it is sufficient to show that $G \times T$ is Hamiltonian connected if $\Delta(T) \leq n-1$.

We argue by induction on $|V(T)|$. The result holds trivially when $|V(T)|=1$, and is 
also true when $|V(T)|=2$ by Lemma 2.2.11. Let $|V(T)|=m(m \geq 3)$ and suppose the result holds for trees of at most $m-1$ vertices. Let $u=\left(x_{u}, y_{u}\right)$ and $v=\left(x_{v}, y_{v}\right)$ be a pair of distinct vertices in $G \times T$. Let $N_{T}\left(y_{u}\right)=\left\{y_{1}, y_{2}, \cdots, y_{k}\right\}(1 \leq k \leq n-1)$. Denote $T_{1}, T_{2}, \cdots, T_{k}$ to be components of $T-y_{u}$ where $y_{i} \in V\left(T_{i}\right)(1 \leq i \leq k)$.

If $y_{u}=y_{v}$, then $x_{u} \neq x_{v}$. Since $(G)_{y_{u}} \cong G$ is Hamiltonian connected, there is a Hamiltonian $(u, v)$-path $P_{y_{u}}$ of $(G)_{y_{u}}$. Denote $P_{y_{u}}=\left(x_{1}, y_{u}\right)\left(x_{2}, y_{u}\right) \cdots\left(x_{n}, y_{u}\right)$ where $x_{1}=x_{u}$ and $x_{n}=x_{v}$. In $G \times T_{i}(1 \leq i \leq k)$, by induction, there is a Hamiltonian $\left(\left(x_{i}, y_{i}\right),\left(x_{i+1}, y_{i}\right)\right)$-path $P_{i}$. Thus

$$
\begin{aligned}
P= & \left(P_{y_{u}}-\left\{\left(x_{i}, y_{u}\right)\left(x_{i+1}, y_{u}\right): 1 \leq i \leq k\right\}\right)+\sum_{i=1}^{k} P_{i}+ \\
& \left\{\left(x_{i}, y_{u}\right)\left(x_{i}, y_{i}\right),\left(x_{i+1}, y_{u}\right)\left(x_{i+1}, y_{i}\right): 1 \leq i \leq k\right\}
\end{aligned}
$$

is a Hamiltonian $(u, v)$-path of $G \times T$.

So we may assume that $y_{u} \neq y_{v}$. Without loss of generality, we may assume that $y_{v} \in V\left(T_{k}\right)$. Choose $x_{0} \in V(G) \backslash\left\{x_{u}, x_{v}\right\}$. Note that $\left(x_{0}, y_{k}\right)$ and $v$ are distinct vertices. By induction, there is a Hamiltonian $\left(u,\left(x_{0}, y_{u}\right)\right)$-path $P^{\prime}$ of $G \times\left(T-T_{k}\right)$ and there is a Hamiltonian $\left(\left(x_{0}, y_{k}\right), v\right)$-path $P^{\prime \prime}$ of $G \times T_{k}$. Thus $P^{\prime}+\left(x_{0}, y_{u}\right)\left(x_{0}, y_{k}\right)+P^{\prime \prime}$ is a Hamiltonian $(u, v)$-path of $G \times T$. This completes the proof of Theorem 2.1.4.

\subsection{Weakly edge-pancyclic Cartesian product}

In this section, we will prove Theorem 2.1.5.

Proof of Theorem 2.1.5 Let $G$ be an edge-pancyclic graph of order $n$ and let $T$ be a tree. The necessary condition follows by Lemma 2.2.12. So it is sufficient to show that $G \times T$ is weakly edge-pancyclic if $\Delta(T) \leq n-1$. By the definition of weakly edge-pancyclic, we actually need to prove the following theorem.

Theorem 2.6.1 Let $G$ be an edge-pancyclic graph of order $n$. Let $T$ be a tree with $\Delta(T) \leq n-1$. Then in $G \times T$, each of the following holds: 
(a) for $\forall y \in V(T), \forall e \in E\left((G)_{y}\right)$ and $\forall l(3 \leq l \leq|V(G \times T)|)$, there is a circuit of length $l$ containing e;

(b) for $\forall x \in V(G), \forall e \in E\left((T)_{x}\right)$ and $\forall l(4 \leq l \leq|V(G \times T)|)$, there is a circuit of length $l$ containing e.

Proof. (a) We use induction on $|V(T)|$. The result holds trivially when $|V(T)|=1$. Let $|V(T)|=m(m \geq 2)$ and suppose the result holds for trees with at most $m-1$ vertices.

Let $y \in V(T)$ and $e \in E\left(G_{y}\right)$ be given. Denote $\left(x_{1}, y\right)$ and $\left(x_{n}, y\right)$ to be the endpoints of $e$. Suppose $N_{T}(y)=\left\{y_{1}, y_{2}, \cdots, y_{k}\right\}$ (where $1 \leq k \leq n-1$ ). Denote $T_{1}, T_{2}, \cdots, T_{k}$ to be components of $T-y$ where $y_{i} \in V\left(T_{i}\right)$ and $\left|V\left(T_{i}\right)\right|=n_{i}(1 \leq i \leq k)$. Note that $\sum_{i=1}^{k} n_{i}=m-1$. We will show that for $\forall l(3 \leq l \leq n * m)$, there is a circuit $C_{l}$ of length $l$ containing $e$ in $G \times T$.

If $3 \leq l \leq n$, since $(G)_{y} \cong G$ is edge-pancyclic, there is a circuit $C_{y}^{l}$ of length $l$ containing $e$ in $(G)_{y}$, which can also be viewed as a circuit $C_{l}$ in $G \times T$. Specially, denote

$$
\begin{gathered}
C_{y}^{n}=\left(x_{1}, y\right)\left(x_{2}, y\right) \cdots\left(x_{n-1}, y\right)\left(x_{n}, y\right), \\
\text { and } C_{y}^{n-1}=\left(x_{1}, y\right)\left(x_{2}^{\prime}, y\right) \cdots\left(x_{n-2}^{\prime}, y\right)\left(x_{n}, y\right) \text {. }
\end{gathered}
$$

If $l=n+1$, then

$$
C_{l}=\left(C_{y}^{n-1}-\left(x_{1}, y\right)\left(x_{2}^{\prime}, y\right)\right)+\left\{\left(x_{1}, y\right)\left(x_{1}, y_{1}\right),\left(x_{1}, y_{1}\right)\left(x_{2}^{\prime}, y_{1}\right),\left(x_{2}^{\prime}, y\right)\left(x_{2}^{\prime}, y_{1}\right)\right\}
$$

So we may assume that $l \geq n+2$. By Lemma 2.2.14, there is a sequence of $k$ integers $\left\{l_{1}, l_{2}, \cdots, l_{k}\right\}$ such that $l=n+\sum_{i=1}^{k} l_{i}$, and $l_{i}$ is either 0 or in the interval $\left[2, n_{i} * n\right]$ (for all $1 \leq i \leq k)$. Let $I_{1}=\left\{i: l_{i} \geq 3\right\}$ and $I_{2}=\left\{j: l_{j}=2\right\}$ be two disjoint subscribe sets. For $\forall i \in I_{1}$, by induction, there is a circuit $C_{T_{i}}^{l_{i}}$ of length $l_{i}$ containing $\left(x_{i}, y_{i}\right)\left(x_{i+1}, y_{i}\right)$ in $G \times T_{i}$. Thus

$$
\begin{aligned}
C_{l}= & \left(C_{y}^{n}-\left\{\left(x_{i}, y\right)\left(x_{i+1}, y\right): i \in I_{1} \cup I_{2}\right\}\right)+ \\
& \sum_{i \in I_{1}}\left(C_{T_{i}}^{l_{i}}-\left(x_{i}, y_{i}\right)\left(x_{i+1}, y_{i}\right)\right)+\sum_{j \in I_{2}}\left\{\left(x_{j}, y_{j}\right)\left(x_{j+1}, y_{j}\right)\right\}+ \\
& \left\{\left(x_{i}, y\right)\left(x_{i}, y_{i}\right),\left(x_{i+1}, y\right)\left(x_{i+1}, y_{i}\right): i \in I_{1} \cup I_{2}\right\} .
\end{aligned}
$$


(b) We use induction on $|V(T)|$. The result holds trivially when $|V(T)|=1$, and it's also true when $|V(T)|=2$ by Lemma 2.2.13. Let $|V(T)|=m(m \geq 3)$ and suppose the result holds for trees with at most $m-1$ vertices. Let $x \in V(G)$ and $e \in E\left(T_{x}\right)$. Denote $\left(x, y_{1}\right)$ and $\left(x, y_{2}\right)$ to be the endpoints of $e$. We will show that for $\forall l(4 \leq l \leq n * m)$, there is a circuit $C_{l}$ of length $l$ containing $e$ in $G \times T$.

Since $G$ is pancyclic, there is a triangle $x z_{1} z_{2}$ in $G$, where $z_{1}, z_{2} \in N_{G}(x)$. Then

$$
C_{4}=\left(x, y_{1}\right)\left(x, y_{2}\right)\left(z_{1}, y_{2}\right)\left(z_{1}, y_{1}\right)
$$

and $C_{5}=\left(x, y_{1}\right)\left(z_{2}, y_{1}\right)\left(z_{1}, y_{1}\right)\left(z_{1}, y_{2}\right)\left(x, y_{2}\right)$.

So we may assume that $l \geq 6$. Let $T_{1}$ and $T_{2}$ be two components of $T-y_{1} y_{2}$ such that $y_{i} \in T_{i}(i=1,2)$. Let $l_{1}=\min \left\{\lfloor l / 2\rfloor, n\left|V\left(T_{1}\right)\right|\right\}$ and $l_{2}=l-l_{1}$. Note that both $l_{1}$ and $l_{2}$ are at least 3 . By result of (a), there is a circuit $C_{i}$ of length $l_{i}$ containing $\left(x, y_{i}\right)\left(z_{1}, y_{i}\right)$ in $G \times T_{i}(i=1,2)$. Thus

$$
\begin{aligned}
C_{l}= & \left(C_{1}-\left(x, y_{1}\right)\left(z_{1}, y_{1}\right)\right)+\left(C_{2}-\left(x, y_{2}\right)\left(z_{1}, y_{2}\right)\right)+ \\
& \left\{\left(x, y_{1}\right)\left(x, y_{2}\right),\left(z_{1}, y_{1}\right)\left(z_{1}, y_{2}\right)\right\} \cdot \square
\end{aligned}
$$

\subsection{Pan-connected Cartesian product}

In this section, we will prove Theorem 2.1.6.

Proof of Theorem 2.1.6 By the definition of pan-connected and Hamiltonian connected, a pan-connected graph is also Hamiltonian connected. Therefore, the necessary condition follows by Theorem 2.1.4. So it is sufficient to show that $G \times T$ is pan-connected if $\Delta(T) \leq n-1$.

We use induction on $|V(T)|$. The result holds trivially when $|V(T)|=1$. Let $|V(T)|=$ $m(m \geq 2)$ and suppose the result holds for trees with at most $m-1$ vertices. Let $u=\left(x_{u}, y_{u}\right)$ and $v=\left(x_{v}, y_{v}\right)$ be two vertices of $G \times T$. Note that $\operatorname{dist}_{G \times T}(u, v)=$ $\operatorname{dist}_{G}\left(x_{u}, x_{v}\right)+\operatorname{dist}_{T}\left(y_{u}, y_{v}\right)$. Suppose $N_{T}\left(y_{u}\right)=\left\{y_{1}, y_{2}, \cdots, y_{k}\right\}$ (where $\left.1 \leq k \leq n-1\right)$. 
Denote $T_{1}, T_{2}, \cdots, T_{k}$ to be components of $T-y_{u}$ where $y_{i} \in V\left(T_{i}\right)$ and $\left|V\left(T_{i}\right)\right|=n_{i}$ $(1 \leq i \leq k)$. Note that $\sum_{i=1}^{k} n_{i}=m-1$. We will show that for $\forall l\left(\operatorname{dist}_{G \times T}(u, v) \leq l \leq\right.$ $n * m-1)$, there is a $(u, v)-$ path $P_{l}$ of length $l$ in $G \times T$.

Case $1 y_{u}=y_{v}$

In this case, $\operatorname{dist}_{G \times T}(u, v)=\operatorname{dist}_{G}\left(x_{u}, x_{v}\right)$. If $\operatorname{dist}_{G \times T}(u, v) \leq l \leq n-1$, since $(G)_{y_{u}} \cong G$ is pan-connected, there is a $(u, v)$-path $P_{y_{u}}^{l}$ of length $l$ in $(G)_{y_{u}}$, which can also be viewed as a $(u, v)$-path $P_{l}$ in $G \times T$. Specially, denote

$$
\begin{gathered}
P_{y_{u}}^{n-1}=\left(x_{1}, y_{u}\right)\left(x_{2}, y_{u}\right) \cdots\left(x_{n-1}, y_{u}\right)\left(x_{n}, y_{u}\right), \\
\text { and } P_{y_{u}}^{n-2}=\left(x_{1}, y_{u}\right)\left(x_{2}^{\prime}, y_{u}\right) \cdots\left(x_{n-2}^{\prime}, y_{u}\right)\left(x_{n}, y_{u}\right),
\end{gathered}
$$

where $x_{1}=x_{u}$ and $x_{n}=x_{v}$.

If $l=n$, then

$$
P_{l}=\left(P_{y_{u}}^{n-2}-\left(x_{1}, y_{u}\right)\left(x_{2}^{\prime}, y_{u}\right)\right)+\left\{\left(x_{1}, y_{u}\right)\left(x_{1}, y_{1}\right),\left(x_{1}, y_{1}\right)\left(x_{2}^{\prime}, y_{1}\right),\left(x_{2}^{\prime}, y_{u}\right)\left(x_{2}^{\prime}, y_{1}\right)\right\} .
$$

If $l=n+1$, then

$$
P_{l}=\left(P_{y_{u}}^{n-1}-\left(x_{1}, y_{u}\right)\left(x_{2}, y_{u}\right)\right)+\left\{\left(x_{1}, y_{u}\right)\left(x_{1}, y_{1}\right),\left(x_{1}, y_{1}\right)\left(x_{2}, y_{1}\right),\left(x_{2}, y_{u}\right)\left(x_{2}, y_{1}\right)\right\}
$$

So we may assume that $n+2 \leq l \leq n * m-1$. By Lemma 2.2.14, there is a sequence of $k$ integers $\left\{l_{1}, l_{2}, \cdots, l_{k}\right\}$ such that $l=n+\sum_{i=1}^{k} l_{i}$, and $l_{i}$ is either 0 or in the interval $\left[2, n_{i} * n\right]$ for $\forall i \in[1, k]$. Let $I=\left\{i: l_{i} \neq 0\right\}$ be a set of subscribes. Choose $i_{0} \in I$. By induction, there is a $\left(\left(x_{i}, y_{i}\right),\left(x_{i+1}, y_{i}\right)\right)$-path $P_{i}^{l_{i}-1}$ of length $l_{i}-1$ in $G \times T_{i}$ for every $i \in I-i_{0}$, and there is a $\left(\left(x_{i_{0}}, y_{i_{0}}\right),\left(x_{i_{0}+1}, y_{i_{0}}\right)\right)$-path $P_{i_{0}}^{l_{i_{0}}}$ of length $l_{i_{0}}$ in $G \times T_{i_{0}}$. Thus

$$
\begin{aligned}
P_{l}= & \left(P^{n-1}-\left\{\left(x_{i}, y\right)\left(x_{i+1}, y\right): i \in I\right\}\right)+ \\
& \sum_{i \in I-i_{0}} P_{i}^{l_{i}-1}+P_{i_{0}}^{l_{i_{0}}}+\left\{\left(x_{i}, y_{u}\right)\left(x_{i}, y_{i}\right),\left(x_{i+1}, y_{u}\right)\left(x_{i+1}, y_{i}\right): i \in I\right\} .
\end{aligned}
$$

\section{Case $2 y_{u} \neq y_{v}$}

Without loss of generality, we may assume that $y_{v} \in V\left(T_{k}\right)$. If $\operatorname{dist}_{G \times T}(u, v) \leq l \leq$ $n+n_{k} * n-1$, by induction, there is a $(u, v)$-path $P_{k}^{l}$ of length $l$ in $G \times\left(y_{u} \cup T_{k}\right)$, which can also be viewed as a $(u, v)-$ path $P_{l}$ in $G \times T$. 
So we may assume that $l \geq n+n_{k} * n$. Choose $z \in N_{G}\left(x_{u}\right) \backslash\left\{x_{v}\right\}$. Note that $\left(z, y_{k}\right)$ and $v$ are distinct vertices. By induction, there is a $\left(u,\left(z, y_{k}\right)\right)$-path $P^{l-n_{k} * n}$ of length $l-n_{k} * n$ in $G \times\left(T-T_{k}\right)$, and there is a $\left(\left(z, y_{k}\right), v\right)$-path $P^{n_{k} * n-1}$ of length $n_{k} * n-1$ in $G \times T_{k}$. Thus $P_{l}=P^{l-n_{k} * n}+\left\{\left(z, y_{u}\right)\left(z, y_{k}\right)\right\}+P^{n_{k} * n-1}$. This completes the proof of Theorem 2.1.6. 


\section{Chapter 3}

\section{On 3-edge-connected Supereulerian Graphs}

\subsection{Introduction}

Graphs in this section may have multiple edges or loops.

A graph $G$ is eulerian if $G$ is connected with $O(G)=\emptyset$, and $G$ is supereulerian if $G$ has a spanning eulerian subgraph. In [3], Boesch et al raised a problem to determine when a graph is supereulerian, and they remarked that such a problem would be a difficult one. In [32], Pulleyblank confirmed the remark by showing that the problem to determine if a graph is supereulerian, even within planar graphs, is NP-complete. For more literature on supereulerian graphs, see Catlin's excellent survey [8] and its supplement [13]. Jaeger and Catlin independently proved the following theorem.

Theorem 3.1.1 (Catlin [7] and Jaeger [20]) Every 4-edge-connected graph is supereulerian.

It is well known that there exist many 3-edge-connected graphs such as the Petersen graph, and any 3-connected cubic graph that does not have a proper 3-edge-coloring 
is not supereulerian. Therefore, it is natural to ask when a 3-edge-connected graph is supereulerian. In [44], Zhan proved the following.

Theorem 3.1.2 (Zhan [44]) Every 3-edge-connected, essentially 7-edge-connected graph is supereulerian.

As seen above, there exist many 3-edge-connected, essentially 4-edge-connected non supereulerian graphs. The following conjecture has been posted in [13].

Conjecture 3.1.3 (Chen and Lai [13]) Every 3-edge-connected, essentially 5-edge-connected graph is supereulerian.

In this chapter, we will investigate when a 3-edge-connected essentially 4-edge-connected graph is supereulerian. Let $\sigma(G)=\min \left\{d_{G}(u)+d_{G}(v) \mid u v \in E(G)\right\}$. The main result of this paper is the following.

Theorem 3.1.4 Let $G$ be a 3-edge-connected, essentially 4-edge-connected graph. If $\sigma(G) \geq 9$, then $G$ is supereulerian.

Since the Pertersen graph is 3-edge-connected, essentially 4-edge-connected with $\sigma(G)=$ 6 , and since the Petersen graph is not supereulerian, we propose the following conjecture.

Conjecture 3.1.5 Let $G$ be a 3-edge-connected, essentially 4-edge-connected graph. If $\sigma(G) \geq 7$, then $G$ is supereulerian.

The line graph of a graph $G$, denoted by $L(G)$, has $E(G)$ as its vertex set, where two vertices in $L(G)$ are adjacent if and only if the corresponding edges in $G$ have at least one vertex in common. In 1986, Thomassen [40] conjectured that every 4-connected line graph is hamiltonian. Zhan [44] verified the Thomassen's conjecture for all 7-connected line graphs. Theorem 3.1.4 has the following corollaries for line graphs. 
Corollary 3.1.6 Every 4-connected, essentially 6-connected line graph with minimum degree at least 7 is hamiltonian.

If a graph is 6-connected, then it must also be 4-connected and essentially 6-connected. So we have the following.

Corollary 3.1.7 Every 6-connected line graph with minimum degree at least 7 is hamiltonian.

Ryjáček [35] introduced the line graph closure of a claw-free graph and used it to show that a claw-free graph $G$ is hamiltonian if and only if it $\operatorname{closure} \operatorname{cl}(G)$ is hamiltonian, where $\operatorname{cl}(G)$ is a line graph. With this argument and using the fact that adding edges will not decrease the connectivity and minimum degree of a graph, the following corollary is obtained.

Corollary 3.1.8 Every 6-connected claw-free graph with minimum degree at least 7 is hamiltonian.

\subsection{Preliminary}

Let $X \subseteq E(G)$ be an edge subset. The contraction $G / X$ is the graph obtained from $G$ by identifying the two ends of each edge in $X$ and then deleting the resulting loops. When $X=\{e\}$, we use $G / e$ for $G /\{e\}$. If $K$ is a subgraph of $G$, then we write $G / K$ for $G / E(K)$.

Theorem 3.2.1 (Zhan [44]) Let $G$ be a 3-edge-connected, essentially 7-edge-connected graph. Then each of the following holds.

(i) For any edge $e \in E(G), F(G-e)=0$.

(ii) For any edges $e, e^{\prime} \subseteq E(G)$ such that $\kappa^{\prime}\left(G-\left\{e, e^{\prime}\right\}\right) \geq 2, F\left(G-\left\{e, e^{\prime}\right\}\right)=0$. 
Corollary 3.2.2 Let $G$ be a 3-edge-connected, essentially 7-edge-connected graph. Then each of the following holds.

(i). For any vertex $v \in D_{3}(G) \cup D_{4}(G), F(G-v)=0$, and so $G$ is collapsible.

(ii). For any vertex $v \in D_{5}(G), F(G-v) \leq 1$. Furthermore, either $G-v$ is collapsible, or $G-v=K_{2}$ and $G$ is $L_{1}$ in Figure 1 .

(iii). For any vertex $v \in D_{6}(G), F(G-v) \leq 2$. Furthermore, either $G-v$ is collapsible, or $G-v=K_{2}$ and $G$ is either $L_{2}$ or $L_{3}$ in Figure 1 , or $G-v=K_{2,6}$ and $G=K_{3,6}$.

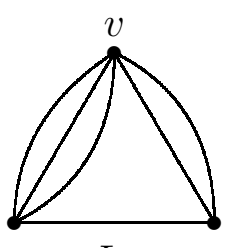

$L_{1}$

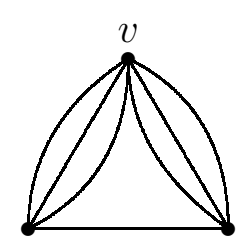

$L_{2}$

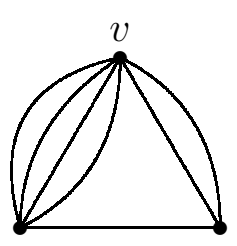

$L_{3}$

Figure 1

Proof. (i) Let $E_{G}(v)=\left\{\begin{array}{ll}\left\{e, e_{1}, e_{2}\right\}, & \text { if } d_{G}(v)=3 \\ \left\{e^{\prime}, e^{\prime \prime}, e_{1}, e_{2}\right\}, & \text { if } d_{G}(v)=4\end{array}\right.$. Since $G$ is essentially 7-edgeconnected, $\kappa^{\prime}\left(G-\left\{e^{\prime}, e^{\prime \prime}\right\}\right) \geq 2$. Let $G_{1}=\left\{\begin{array}{ll}G-e, & \text { if } d_{G}(v)=3 \\ G-\left\{e^{\prime}, e^{\prime \prime}\right\}, & \text { if } d_{G}(v)=4\end{array}\right.$. By Theorem 3.2.1, $F\left(G_{1}\right)=0$. We assume that $T_{1}, T_{2}$ are two edge-disjoint spanning trees of $G_{1}$. Without loss of generality, we also assume that $e_{1} \in T_{1}, e_{2} \in T_{2}$. Then $T_{1}-v, T_{2}-v$ are two edge-disjoint spanning trees of $G-v$.

(ii) Let $E_{G}(v)=\left\{e^{\prime}, e^{\prime \prime}, e_{1}, e_{2}, e_{3}\right\}$. Clearly, $\kappa^{\prime}\left(G-\left\{e^{\prime}, e^{\prime \prime}\right\}\right) \geq 2$. By Theorem 3.2.1(ii), $F\left(G-\left\{e^{\prime}, e^{\prime \prime}\right\}\right)=0$. We assume that $T_{1}, T_{2}$ are two edge-disjoint spanning trees of $G-\left\{e^{\prime}, e^{\prime \prime}\right\}$ such that $e_{1} \in T_{1}$ and $e_{2} \in T_{2}$. If $e_{3} \notin E\left(T_{1}\right) \cup E\left(T_{2}\right), F(G-v)=0$. Otherwise, $F(G-v) \leq 1$. By Theorem 2.1(iii), either $G-v$ is collapsible or the c-reduction of $G-v$ is a $K_{2}$. If the c-reduction of $G-v$ is a $K_{2}$, let $V\left(K_{2}\right)=\left\{w_{1}, w_{2}\right\}$. Since $G$ is essentially 7-edge-connected and 3-edge-connected, $\left|V\left(\mathcal{C P}\left(w_{1}\right)\right)\right|=\left|V\left(\mathcal{C P}\left(w_{2}\right)\right)\right|=1$. Thus $G$ is $L_{1}$ in Figure 1.

(iii) Let $E_{G}(v)=\left\{e^{\prime}, e^{\prime \prime}, e_{1}, e_{2}, e_{3}, e_{4}\right\}$. Then $\kappa^{\prime}\left(G-\left\{e^{\prime}, e^{\prime \prime}\right\}\right) \geq 2$. By Theorem 3.2.1(ii), $F\left(G-\left\{e^{\prime}, e^{\prime \prime}\right\}\right)=0$. We assume that $T_{1}, T_{2}$ are two edge-disjoint spanning trees of $G-\left\{e^{\prime}, e^{\prime \prime}\right\}$ such that $e_{1} \in T_{1}$ and $e_{2} \in T_{2}$. If $e_{3}, e_{4} \notin E\left(T_{1}\right) \cup E\left(T_{2}\right), F(G-v)=0$. 
Otherwise, $F(G-v)=1$ or 2. By Theorem 1.2.4 (iii), either $G-v$ is collapsible or the c-reduction of $G-v$ is a $K_{2}$ or a $K_{2, t}(t \geq 1)$. Let $G^{\prime}$ be the c-reduction of $G-v$.

If $G^{\prime}=K_{2}$, let $V\left(G^{\prime}\right)=\left\{w_{1}, w_{2}\right\}$. Since $G$ is essentially 7-edge-connected and 3-edgeconnected, $\left|V\left(\mathcal{C P}\left(w_{1}\right)\right)\right|=\left|V\left(\mathcal{C P}\left(w_{2}\right)\right)\right|=1$. Thus $G$ is either $L_{2}$ or $L_{3}$ in Figure 1 . Next we assume that $G^{\prime}=K_{2, t}(t \geq 1)$. Let $V\left(G^{\prime}\right)=\left\{u_{1}, u_{2}, w_{1}, w_{2}, \cdots, w_{t}\right\}$ with $d_{G^{\prime}}\left(u_{i}\right)=$ $t(i=1,2)$ and $d_{G^{\prime}}\left(w_{j}\right)=2(j=1, \cdots, t)$. If $\left[v, \mathcal{C P}\left(w_{j}\right)\right]=0$, then $\left|V\left(\mathcal{C P}\left(w_{j}\right)\right)\right| \geq 2$ since $\kappa^{\prime}(G) \geq 3$. Thus $\left[\mathcal{C P}\left(w_{j}\right), G-\mathcal{C P}\left(w_{j}\right)\right]$ is an essential 2-edge cut in $G$, a contradiction. Thus $\left\|v, \mathcal{C P}\left(w_{j}\right)\right\| \geq 1$. As $d_{G}(v)=6, t \leq 6$.

Assume that $\left\|v, \mathcal{C P}\left(w_{j}\right)\right\| \geq 2$. If $t \geq 2,\left(\left[\mathcal{C P}\left(w_{j}\right), G-\mathcal{C P}\left(w_{j}\right)\right] \cup[v, G-v]\right)-\left[v, \mathcal{C P}\left(w_{j}\right)\right]$ is an essential edge cut of size at most 6 in $G$; if $t=1$, then $\left\|v, \mathcal{C P}\left(u_{1}\right)\right\|=\left\|v, \mathcal{C P}\left(u_{2}\right)\right\| \geq 2$ since $G$ is 3-edge-connected. Thus $\left\|v, \mathcal{C P}\left(u_{1}\right)\right\|=\left\|v, \mathcal{C P}\left(u_{2}\right)\right\|=\left\|v, \mathcal{C P}\left(w_{1}\right)\right\|=2$, and so $\left[\mathcal{C P}\left(w_{1}\right) \cup \mathcal{C P}\left(u_{1}\right), G-\left(\mathcal{C P}\left(w_{1}\right) \cup \mathcal{C P}\left(u_{1}\right)\right)\right]$ is an essential 5 -edge cut. It contradicts the hypothesis that $G$ is essentially 7 -edge-connected. So $\left\|v, \mathcal{C P}\left(w_{j}\right)\right\|=1$. Since $G$ is again essentially 7 -edge-connected, $\left|V\left(\mathcal{C P}\left(w_{i}\right)\right)\right|=1$.

If $t \leq 5$, then $\left.\| v, \mathcal{C P}\left(u_{1}\right) \cup \mathcal{C P}\left(u_{2}\right)\right\} \| \geq 1$. Without loss of generality, we assume that $\left\|v, \mathcal{C P}\left(u_{1}\right)\right\| \geq\left\|v, \mathcal{C P}\left(u_{2}\right)\right\|$. Then $\left\|\left\{w_{1}\right\} \cup \mathcal{C P}\left(u_{2}\right), G-\left(\left\{w_{1}\right\} \cup \mathcal{C P}\left(u_{2}\right)\right)\right\| \leq 6$, contrary to the hypothesis that $G$ is essentially 7-edge-connected. So $t=6$. Thus $v w_{j} \in$ $E(G)(j=1, \cdots, 6)$. Since $G$ is essential 7-edge-connected again, $\left|V\left(\mathcal{C P}\left(u_{i}\right)\right)\right|=1(i=$ $1,2)$. Therefore, $G=K_{3,6}$.

\subsection{Proofs of Theorems}

In this section, we will prove Theorem 3.1.4 and Corollary 3.1.6.

Proof of Theorem 3.1.4. By contradiction, we assume that Theorem 3.1.4 is false. Then there is a graph $G$ satisfying the hypothesis of Theorem 3.1.4 but $G$ is not supereulerian. Choose $G$ such that

(1) $G$ is a counterexample of Theorem 3.1.4; 
(2) subject to (1), $|V(G)|$ is minimized.

If $G$ is essentially 7-edge-connected, then, by Theorem 3.2.1, $F(G)=0$. By Theorem 1.2.4(iv), $G$ is supereulerian, contrary to (1). Thus $G$ has an essential edge cut $X$ with $4 \leq|X| \leq 6$. Let $G_{1}$ and $G_{2}$ denote two nontrivial connected components of $G-X$ with $\left|V\left(G_{1}\right)\right| \leq\left|V\left(G_{2}\right)\right|$. We choose $G$ and $X$ so that

(3) subject to (1) and (2), $\left|V\left(G_{1}\right)\right|$ is as small as possible.

Let $G_{1}^{\prime}=G / G_{2}$ and $G_{2}^{\prime}=G / G_{2}$, and let $v_{G_{1}}$ and $v_{G_{2}}$ denote the vertices onto which $G_{1}$ and $G_{2}$ are contracted, respectively (see Figure 2). Then $X=\left[v_{G_{2}}, G_{1}^{\prime}-v_{G_{2}}\right]_{G_{1}^{\prime}}$ and $X=$ $\left[v_{G_{1}}, G_{2}^{\prime}-v_{G_{1}}\right]_{G_{2}^{\prime}}$. As contraction will not decrease edge-connectivity and essential edgeconnectivity, both $G_{1}^{\prime}$ and $G_{2}^{\prime}$ are also 3-edge-connected and essentially 4-edge-connected. By (2), $G_{2}^{\prime}$ is supereulerian.

If $G_{1}^{\prime}$ has an essential edge cut $Y$ with $|Y| \in\{4,5,6\}$, let $G_{11}$ and $G_{12}$ be the components of $G-Y$. Also, we assume that $v_{G_{2}} \in V\left(G_{12}\right)$. Thus $Y$ is also an essential edge cut of $G$ such that $G_{11}$ and $G\left[V\left(G_{12}\right) \cup V\left(G_{2}\right)\right]$ are nontrivial components of $G-Y$, contrary to (3). So $G_{1}^{\prime}$ is 3-edge-connected and essentially 7-edge-connected.

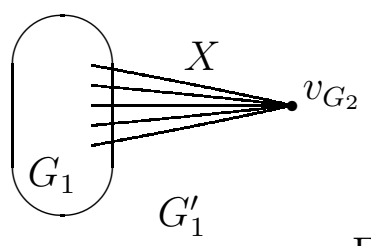

Figure 2.

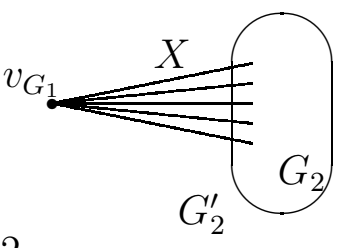

Note that $d_{G_{1}^{\prime}}\left(v_{G_{2}}\right)=|X| \leq 6$ and $G_{1}^{\prime}-v_{G_{2}}=G_{1}$. By Corollary 3.2.2, either $G_{1}$ is collapsible, or $G_{1}=K_{2}$, or $G_{1}=K_{2,6}$. Note that $G_{2}^{\prime}$ is supereulerian, and $G_{2}^{\prime}=G / G_{1}$. If $G_{1}$ is collapsible, then $G$ is supereulerian by Theorem 1.2.1(viii), contrary to (1). So we have either $G_{1}=K_{2}$ or $G_{1}=K_{2,6}$.

Claim 1. $\kappa^{\prime}\left(G_{1}\right) \geq 2$. Therefore, $G_{1}=K_{2,6}$ and $G_{1}^{\prime}=K_{3,6}$.

By contradiction, we assume that $\kappa^{\prime}\left(G_{1}\right) \leq 1$. If $G_{1}$ is not connected, let $G_{11}$ and 
$G_{12}$ be the components of $G_{1}$. Since $G$ is essentially 4-edge-connected with $\kappa^{\prime}(G) \geq 3$ and $|X| \leq 6$, we have $\left[G_{2}, G_{1 i}\right]=3,\left|E\left(G_{1 i}\right)\right|=0$, and $\left|V\left(G_{1 i}\right)\right|=1$ for $i=1,2$. This contradicts the hypothesis that $X$ is an essential edge cut. Thus $G_{1}$ must be connected, and so $G_{1}$ has a cut edge $e$. Let $G_{11}^{\prime}$ and $G_{12}^{\prime}$ be the two components of $G_{1}-e$. Then $\left[G_{11}^{\prime}, G-G_{11}^{\prime}\right] \cup\left[G_{12}^{\prime}, G-G_{12}^{\prime}\right]=X \cup\{e\}$ and $\left[G_{11}^{\prime}, G-G_{11}^{\prime}\right] \cap\left[G_{12}^{\prime}, G-G_{12}^{\prime}\right]=\{e\}$. It follows that $\left\|G_{11}^{\prime}, G-G_{11}^{\prime}\right\|+\left\|G_{12}^{\prime}, G-G_{12}^{\prime}\right\|=|X \cup\{e\}|+1 \leq 8$. Since $\kappa^{\prime}(G) \geq 3$, $\max \left\{\left\|G_{11}^{\prime}, G-G_{11}^{\prime}\right\|,\left\|G_{12}^{\prime}, G-G_{12}^{\prime}\right\|\right\} \leq 5$. By (3), neither $G_{11}^{\prime}$ nor $G_{12}^{\prime}$ can have an edge, and so we may assume that $V\left(G_{11}^{\prime}\right)=\{u\}$ and $V\left(G_{12}^{\prime}\right)=\{v\}$. Thus $d_{G}(u)+d_{G}(v) \leq 8$, contrary to the hypothesis that $\sigma(G) \geq 9$. This proves Claim 1 .

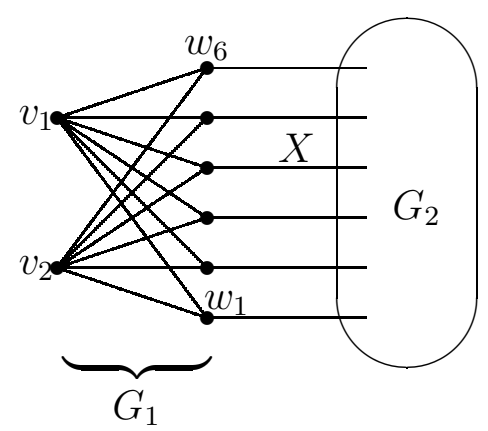

Figure 3 .

By Claim $1, G_{1}=K_{2,6}$ and $G_{1}^{\prime}=K_{3,6}$. Thus $|X|=6$. Let $W=\left\{w_{1}, w_{2}, \cdots, w_{6}\right\}$ and $\left\{v_{1}, v_{2}\right\}$ be the sets of vertices of degree 2 and degree 6 in $G_{1}$, respectively (see Figure 3 ). Note that $G_{2}^{\prime}$ is supereulerian. Let $T$ be a spanning eulerian subgraph of $G_{2}^{\prime}$, and $H=G[E(T)]$.

Assume $|V(H) \cap W|=6$. If $T-v_{G_{1}}$ is not connected in $G_{2}^{\prime}$, then $H$ is not connected in $G$. Let $H_{1}, \cdots, H_{s}$ be the components of $H$, where $s \in\{2,3\}$. Thus there is an $H_{i}$, say $H_{1}$, such that $\left|V\left(H_{1}\right) \cap W\right|=2$. Without loss of generality, we assume that $V\left(H_{1}\right) \cap W=\left\{w_{1}, w_{2}\right\}$. Then $H^{\prime}=G\left[E(H) \cup\left\{v_{1} w_{2}, v_{1} w_{4}, v_{1} w_{5}, v_{1} w_{6}, v_{2} w_{1}, v_{2} w_{3}\right\}\right]$ is a spanning eulerian subgraph of $G$, contrary to (1). If $T-v_{G_{1}}$ is connected in $G_{2}^{\prime}$, then $H$ is also connected in $G$. Thus $H^{\prime}=G\left[E(H) \cup\left\{v_{1} w_{2}, v_{1} w_{4}, v_{1} w_{5}, v_{1} w_{6}, v_{2} w_{1}, v_{2} w_{3}\right\}\right]$ is also a spanning eulerian subgraph of $G$, contrary to (1).

Assume $|V(H) \cap W|=4$. If $T-v_{G_{1}}$ is not connected in $G_{2}^{\prime}$, then $H$ is not connected in 
$G$. Let $H_{1}, H_{2}$ be the components of $H$. Assume that $V\left(H_{1}\right) \cap W=\left\{w_{1}, w_{2}\right\}$ and $V\left(H_{2}\right) \cap$ $W=\left\{w_{3}, w_{4}\right\}$. Then $H^{\prime}=G\left[E(H) \cup\left\{v_{1} w_{1}, v_{1} w_{4}, v_{1} w_{5}, v_{1} w_{6}, v_{2} w_{2}, v_{2} w_{3}, v_{2} w_{5}, v_{2} w_{6}\right\}\right]$ is a spanning eulerian subgraph of $G$, contrary to (1). If $T-v_{G_{1}}$ is connected in $G_{2}^{\prime}$, then $H^{\prime}=G\left[E(H) \cup\left\{v_{1} w_{1}, v_{1} w_{4}, v_{1} w_{5}, v_{1} w_{6}, v_{2} w_{2}, v_{2} w_{3}, v_{2} w_{5}, v_{2} w_{6}\right\}\right]$ is also a spanning eulerian subgraph of $G$, contrary to (1).

If $|V(H) \cap W|=2$, without loss of generality, we assume that $V(H) \cap W=\left\{w_{1}, w_{2}\right\}$. Then $H^{\prime}=G\left[E(H) \cup\left\{v_{1} w_{1}, v_{1} w_{2}, v_{1} w_{3}, v_{1} v_{4}, v_{1} w_{5}, v_{1} w_{6}, v_{2} w_{3}, v_{2} w_{4}, v_{2} w_{5}, v_{2} w_{6}\right\}\right]$ is a spanning eulerian subgraph of $G$, contrary to (1). This completes the proof of Theorem 3.1.4.

In order to prove Corollary 3.1.6, we need the following lemmas.

Lemma 3.3.1 (Shao [38]) Let $G$ be a connected, essentially 3-edge-connected graph.

(i). The core graph $G_{0}$ is uniquely defined, and $\kappa^{\prime}\left(G_{0}\right) \geq 3$.

(ii). If $G_{0}$ is supereulerian, then $L(G)$ is hamiltonian.

Proof of Corollary 3.1.6. Let $L(G)$ be a 4-connected, essentially 6-connected line graph with $\delta(L(G)) \geq 7$. Then $G$ is essentially 4-edge-connected with $\sigma(G) \geq 9$. Let $G_{0}$ be the core of $G$. Then $G_{0}$ is 3-edge-connected, and essentially 4-edge-connected. Thus, $D_{3}(G)=D_{3}\left(G_{0}\right)$.

Let $u v \in E\left(G_{0}\right)$ with $d_{G_{0}}(u)+d_{G_{0}}(v) \leq 8$. If $u v \notin E(G)$, then both $u$ and $v$ are adjacent to a vertex of degree 2 in $G$. Thus $d_{G_{0}}(u)=d_{G}(u) \geq 9-2=7$ and $d_{G_{0}}(v)=d_{G}(v) \geq 9-2=7$, and so $d_{G_{0}}(u)+d_{G_{0}}(v) \geq 14$, contrary to the assumption that $d_{G_{0}}(u)+d_{G_{0}}(v) \leq 8$. So $u v \in E(G)$ and $d_{G}(u)+d_{G}(v) \geq 9$. As $d_{G_{0}}(u)+d_{G_{0}}(v) \leq 8$, one of $u$ or $v$ (say $u$ ) must be adjacent to some vertex of degree 1 in $G$. Since $D_{3}(G)=D_{3}\left(G_{0}\right)$, we have $d_{G_{0}}(u) \geq 4$. As $G_{0}$ is 3 -edge-connected and $d_{G_{0}}(u)+d_{G_{0}}(v) \leq 8$, we have $d_{G_{0}}(u) \leq 5$, and so $d_{G_{0}}(u) \in\{4,5\}$. Since $u$ is adjacent to some vertex of degree 1 in $G$ and $\sigma(G) \geq 9, u$ must be adjacent to at least three vertices of degree 1 in $G$. Thus $\left[u, G_{0}-u\right]_{G_{0}}$ is an essential vertex cut of size 4 or 5 in $L(G)$, contrary to the hypothesis that $L(G)$ is essentially 6-connected. So $\sigma\left(G_{0}\right) \geq 9$. By Theorem 3.1.4, $G_{0}$ has a spanning eulerian subgraph. By Lemma 3.3.1, $L(G)$ is hamiltonian. 


\section{Chapter 4}

\section{4-connected line graph of a $P 3 D$ graph}

\subsection{Introduction}

In [40], Thomassen conjectured that every 4-connected line graph is hamiltonian. In [31], Matthews and Sumner conjectured that every 4-connected claw-free graph is hamiltonian. Ryjáček [35] discovered the line graph closure of claw-free graphs, and showed that the above mentioned conjectures by Thomassen and by Matthews and Sumner are in fact equivalent. Recently, Ryjáček and Vrána further conjectured the following:

Conjecture 4.1.1 (Ryjáček and Vrána, [37]) Every 4-connected claw-free graph is hamiltonian connected.

In 1986, Zhan proved a sufficient condition involving the edge-connectivity $\kappa^{\prime}(G)$ for $L(G)$ to be hamiltonian-connected.

Theorem 4.1.2 (Zhan, [43]) If $G$ is a 4-edge-connected graph, then the line graph $L(G)$ is hamiltonian connected. 
By the spanning tree packing theorem of Tutte and Nash-Williams, it is known that every 4-edge-connected graph has two edge-disjoint spanning trees. Thus the following extends Zhan's theorem.

Theorem 4.1.3 (Catlin and Lai, Corollary $4 A$ in [12]) If $G$ has two edge-disjoint spanning trees, the the line graph $L(G)$ is hamiltonian-connected if and only if $\kappa(L(G)) \geq 3$.

A number of former researches have also investigated the hamiltonicity of the line graph of claw-free graphs. For example, it has been shown that (Corollary 3.9, [14]) every 4-connected line graph of a claw free graph is hamiltonian. Kriesell presented a stronger result.

Theorem 4.1.4 (Kriesell, [23]) Every 4-connected line graph of a claw free graph is hamiltonian connected.

Ryjáček ([36]) introduced the almost claw free graphs. A graph $G$ is almost claw free if the vertices that are centers of claws in $G$ are independent and if the neighborhoods of the center of each claw in $G$ is 2 -dominated (having 2 vertices in the neighborhoods of the center adjacent to other neighbors). By definition, every claw free graph is an almost claw free graph.

Theorem 4.1.5 (Corollary 1.5 in [27]) Every 4-connected line graph of an almost claw free graph is hamiltonian-connected.

For vertices $x, y \in V(G)$ with $\operatorname{dist}_{G}(x, y)=2$, define

$$
J_{G}(x, y)=\left\{u \in N_{G}(x) \cap N_{G}(y): N_{G}[u] \subseteq N_{G}[x] \cup N_{G}[y]\right\},
$$

and

$$
\begin{aligned}
J_{G}^{\prime}(x, y)= & \left\{u \in N_{G}(x) \cap N_{G}(y): \text { if } v \in N_{G}(u)-\left(N_{G}[x] \cup N_{G}[y]\right),\right. \text { then } \\
& \left.N_{G}(x) \cup N_{G}(y) \cup N_{G}(u)-\{x, y, v\} \subseteq N_{G}(v)\right\} .
\end{aligned}
$$


Once again, when the graph $G$ is understood from the context, the subscript $G$ will be omitted.

Ainouche ([1]) introduced the quasi claw-free graphs. A graph $G$ is quasi claw-free if $J_{G}(x, y) \neq \emptyset$ for any $x, y \in V(G)$ with $\operatorname{dist}_{G}(x, y)=2$. Broersma and Vumer [5] recently introduced the $P_{3}$-dominating graphs. A graph $G$ is $P_{3}$-dominating (P3D for short) if $J_{G}(x, y) \cup J^{\prime}(x, y) \neq \emptyset$ for any $x, y \in V(G)$ with $\operatorname{dist}_{G}(x, y)=2$. By definitions, every claw-free graph is quasi claw-free; and every quasi claw-free graph is P3D. A recent result also extends the above mentioned theorem by Kriesell.

Theorem 4.1.6 (Theorem 1.1 in [28]) Every 4-connected line graph of a quasi claw free graph is hamiltonian-connected.

The main purpose of this paper is to investigate whether these theorems can be extended to the line graphs of $P_{3}$-dominating graphs. We obtained the following theorem.

Theorem 4.1.7 Every 4-connected line graph of a $P_{3}$-dominating graph is hamiltonianconnected.

\subsection{Preliminaries}

A graph is 4-cycle-connected if for every pair of distinct edges $e^{\prime}, e^{\prime \prime} \in E(G), G$ has a sequence of cycles $C_{1}, C_{2}, \cdots, C_{t}(t \geq 1)$, referred as a 4-cycle $\left(e^{\prime}, e^{\prime \prime}\right)$-chain, such that $(4 \mathrm{C} 1) e^{\prime} \in E\left(C_{1}\right)$ and $e^{\prime \prime} \in E\left(C_{t}\right)$;

(4C2) for each $i$ with $1 \leq i \leq t,\left|E\left(C_{i}\right)\right| \leq 4$; and

(4C3) if $t \geq 2$, then $\forall i$ with $1 \leq i \leq t-1, E\left(C_{i}\right) \cap E\left(C_{i+1}\right) \neq \emptyset$.

Lemma 4.2.1 Suppose that $G$ is a 4-cycle-connected graph. Then $G$ is collapsible if and only if $G$ has a nontrivial collapsible subgraph. 
Proof It suffices to prove that if $G$ has a nontrivial collapsible subgraph $H$, then $G$ is collapsible. Thus we assume that $G$ has a nontrivial collapsible subgraph $H$. If $H$ spans $G$, then $G / H \cong K_{1}$ is also collapsible. It follows by Theorem 1.2.1(i) that $G$ is also collapsible. Thus we assume that $H$ is a maximal collapsible subgraph of $G$, and that $V(G)-V(H) \neq \emptyset$. Since $G$ is 4-cycle connected, $G$ is also connected, and so there must be an edge $e^{\prime} \in E(G)$ incident with a vertex in $V(H)$ and a vertex in $V(G)-V(H)$. Since $H$ is nontrivial, $\exists e^{\prime \prime} \in E(H)$. Since $G$ is 4-cycle-connected, $G$ has a 4-cycle $\left(e^{\prime}, e^{\prime \prime}\right)$-chain $C_{1}, C_{2}, \cdots, C_{t}$, satisfying (4C1)-(4C3). If we choose $e^{\prime}$ and $e^{\prime \prime}$ so that $t$ is smallest, then we may assume that $t=1$ and $e^{\prime}$ and $e^{\prime \prime}$ are in a cycle $C_{1}$ of length at most 4 . Since $e^{\prime \prime} \in E(H),\left(H \cup C_{1}\right) / H$ is a cycle of length at most 3. By Theorem 1.2.1(ii) and (i), $H \cup C_{1}$ is also collapsible, contrary to the assumption that $H$ is a maximally collapsible subgraph of $G$. Thus we must have $G=H$, and so $G$ is collapsible.

Corollary 4.2.2 Let $n \geq 3$ be an integer. Every $K_{3, n}$ is collapsible.

Proof Every $K_{3, n}$ is 4-cycle-connected and contains a collapsible subgraph $K_{3,3}-e$ (see Theorem 1.2.1(vii)), and so by Lemma $4.2 .1, K_{3, n}$ is collapsible.

We say that an edge $e \in E(G)$ is subdivided when it is replaced by a path of length 2 whose internal vertex, denoted by $v(e)$, has degree 2 in the resulting graph. The process of taking an edge $e$ and replacing it by that length 2 path is called subdividing $e$. For a graph $G$ and edges $e^{\prime}, e^{\prime \prime} \in E(G)$, let $G\left(e^{\prime}\right)$ denote the graph obtained from $G$ by subdividing $e^{\prime}$, and let $G\left(e^{\prime}, e^{\prime \prime}\right)$ denote the graph obtained from $G$ by subdividing both $e^{\prime}$ and $e^{\prime \prime}$. Then,

$$
V\left(G\left(e^{\prime}, e^{\prime \prime}\right)\right)-V(G)=\left\{v\left(e^{\prime}\right), v\left(e^{\prime \prime}\right)\right\}
$$

The above definitions imply the following lemma.

Lemma 4.2.3 For a graph $G$ and edges $e^{\prime}, e^{\prime \prime} \in E(G)$, each of the following holds.

(i) if $G\left(e^{\prime}, e^{\prime \prime}\right)$ has a spanning $\left(v\left(e^{\prime}\right), v\left(e^{\prime \prime}\right)\right)$-trail, then $G$ has a spanning $\left(e^{\prime}, e^{\prime \prime}\right)$-trail.

(ii) if $G\left(e^{\prime}, e^{\prime \prime}\right)$ has a dominating $\left(v\left(e^{\prime}\right), v\left(e^{\prime \prime}\right)\right)$-trail, then $G$ has a dominating $\left(e^{\prime}, e^{\prime \prime}\right)$ trail. 
Lemma 4.2.4 Let $G$ be a graph and $G^{\prime}=G-D_{1}(G)$. If $\kappa(L(G)) \geq 3$ and $L(G)$ is not complete, then

(i) $G^{\prime}$ is nontrivial and $\delta\left(G^{\prime}\right) \geq \kappa^{\prime}\left(G^{\prime}\right) \geq 2$.

(ii) $G_{0}$ in nontrivial and $\delta\left(G_{0}\right) \geq \kappa^{\prime}\left(G_{0}\right) \geq 3$.

(iii) for $v \in V(G)$ with $d_{G}(v)=1$ or $d_{G}(v)=2, N_{G}(v) \subseteq V\left(G_{0}\right)$.

Lemma 4.2.5 Let $G$ be a graph such that $\kappa(G) \geq 3$ and $L(G)$ is not complete and let $G_{0}$ be the core of $G$. If $G_{0}\left(e^{\prime}, e^{\prime \prime}\right)$ has a spanning $\left(v\left(e^{\prime}\right), v\left(e^{\prime \prime}\right)\right)$-trail for any $e^{\prime}, e^{\prime \prime} \in E\left(G_{0}\right)$, then for any $e^{\prime}, e^{\prime \prime} \in E(G), G\left(e^{\prime}, e^{\prime \prime}\right)$ has a dominating $\left(v\left(e^{\prime}\right), v\left(e^{\prime \prime}\right)\right)$-trail.

Proof Let $e^{\prime}, e^{\prime \prime} \in E(G)$. If $e^{\prime} \in E\left(G_{0}\right)$, let $f^{\prime}=e^{\prime}$; if $e^{\prime}$ is incident with a vertex of degree 2 , let $f^{\prime}$ be the corresponding new edge in $G_{0}$; if $e^{\prime}$ is incident to a vertex of degree 1 , let $f^{\prime}$ be any edge in $G_{0}$ incident with the other vertex incident with $e^{\prime}$. Similarly we define $f^{\prime \prime}$. Then a spanning $\left(v\left(f^{\prime}\right), v\left(f^{\prime \prime}\right)\right)$-trail in $G_{0}\left(f^{\prime}, f^{\prime \prime}\right)$ can be adjusted to a dominating $\left(v\left(e^{\prime}\right), v\left(e^{\prime \prime}\right)\right)$-trail in $G$.

Lemma 4.2.6 (Proposition 2.2 of [27])Let $G$ be a graph with $|E(G)| \geq 3$. Then $L(G)$ is hamiltonian connected if and only if for any pair of edges $e^{\prime}, e^{\prime \prime} \in E(G)$, G has a dominating $\left(e^{\prime}, e^{\prime \prime}\right)$-trail.

Theorem 4.2.7 (Lemma 3.1 of [27]) Let $G$ be a 3-edge-connected graph without loops, $v, v_{1}, u_{1}, u_{2} \in V(G)$ be such that $d_{G}\left(v_{1}\right)=3$ and $N_{G}\left(v_{1}\right)=\left\{v, u_{1}, u_{2}\right\}$, and for an integer $k \geq 1$ let $X^{\prime}=\left\{u_{1} u_{2}, u_{1} v_{i}, u_{2} v_{i}: 1 \leq i \leq k\right\}$ be an edge subset of $G$ and $W=G\left[X^{\prime}\right]$.

Then each of the following holds.

(i) If $\left(G-v v_{1}\right) / W$ is nontrivial and $\tau\left(\left(G-v v_{1}\right) / W\right) \geq 2$, then $\tau(G) \geq 2$.

(ii) If $G / W=K_{1}$, then $\tau(G) \geq 2$.

Theorem 4.2.8 (Theorem 3.3 of [27]) Let $G$ be a graph with $\kappa^{\prime}(G) \geq 3$. If every 3-edgecut of $G$ has at least one edge in a 2-cycle or 3-cycle of $G$, then the graph $G\left(e^{\prime}, e^{\prime \prime}\right)$ is collapsible for any $e^{\prime}, e^{\prime \prime} \in E(G)$. 


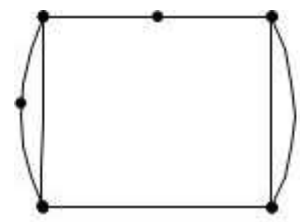

$\mathrm{H}_{1}$

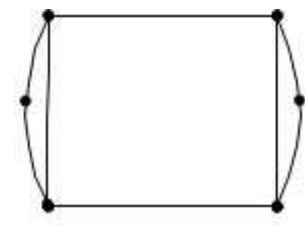

$\mathrm{H}_{2}$

Figure 4.2.1

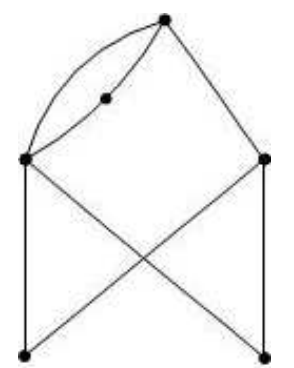

$\mathrm{H}_{3}$

Figure 4.2.2

Lemma 4.2.9 Let $H_{1}$ and $H_{2}$ be graphs depicted as Fig 4.2.1. Then both $H_{1}$ and $H_{2}$ are collapsible.

Proof Note that $H_{1}$ contains a $C_{3}$ and a $C_{2}$. Since $H_{1} /\left(C_{2} \cup C_{3}\right) \cong C_{3}$, by Theorem 1.2.1 (i) and (ii), $H_{1}$ is collapsible.

Since $F\left(H_{2}\right)=0$, by Theorem 1.2.4 (ii), $H_{2}$ is collapsible.

Lemma 4.2.10 Let $H_{3}$ be a graph depicted as Fig 4.2.2. Then $H_{3}$ is collapsible.

Proof Note that $H_{3}$ contains a $C_{3}$. Note $H_{3} / C_{3}$ is isomorphic to $K_{4}-e$, which is 2edge-connected. Since $F\left(H_{3} / C_{3}\right)=1$, by Theorem 1.2.4(ii), $H_{3} / C_{3}$ is collapsible. Thus by Theorem 1.2.1 (i) and (ii), $H_{3}$ is collapsible. 


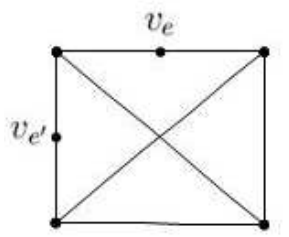

$K_{4}\left(e, e^{\prime}\right)$

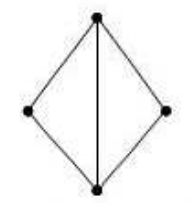

$K_{4}\left(e, e^{\prime}\right) / C_{3}$

(a)

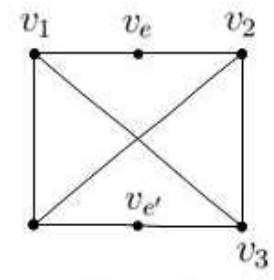

$K_{4}\left(e, e^{\prime}\right)$

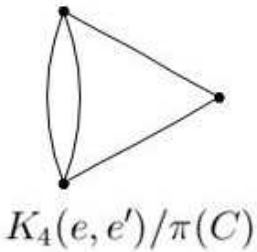

(b)

Figure 4.2.3

Lemma 4.2.11 For $\forall e, e^{\prime} \in E\left(K_{4}\right), K_{4}\left(e, e^{\prime}\right)$ is collapsible.

Proof If $e$ and $e^{\prime}$ have a common endpoint (Fig $4.2 .3(\mathrm{a})$ ), then $K_{4}\left(e, e^{\prime}\right)$ has a $C_{3}$. $K_{4}\left(e, e^{\prime}\right) / C_{3}$ is isomorphic to $K_{4}-e$, which is 2-edge-connected. Since $F\left(K_{4}\left(e, e^{\prime}\right) / C_{3}\right)=1$, by Theorem 1.2.4(ii), $K_{4}\left(e, e^{\prime}\right) / C_{3}$ is collapsible. Thus by Theorem 1.2.1 (i) and (ii), $K_{4}\left(e, e^{\prime}\right)$ is collapsible. So we may assume that $e_{1}$ and $e_{2}$ is a matching of $K_{4}$ (Fig 4.2.3 (b)). Note that $C=v_{1} v_{e} v_{2} v_{3}$ is a 4-circuit of $K_{4}\left(e, e^{\prime}\right)$. Since $K_{4}\left(e, e^{\prime}\right) / \pi(C)$ has a spanning 3-circuit, by Theorem 1.2.1 (ii), the reduction of $K_{4}\left(e, e^{\prime}\right) / \pi(C)$ is $K_{1}$, which means $K_{4}\left(e, e^{\prime}\right) / \pi(C)$ is collapsible. Hence, by Theorem 1.2.6 (i), $K_{4}\left(e, e^{\prime}\right)$ is collapsible.

Lemma 4.2.12 Let $H_{4}$ be a graph depicted as Fig 4.2.4. Then $H_{4}$ is collapsible.

Proof Let $K$ be the induced subgraph of $\left\{v_{1}, v_{2}, v_{3}, v_{4}\right\}$ in $H_{4}$. By Theorem 1.2.4(ii), $K$ is collapsible. Since $H_{4} / K \cong C_{3}$, by Theorem 1.2.1 (i) and (ii), $H_{4}$ is collapsible. 


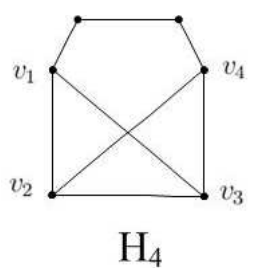

Figure 4.2 .4

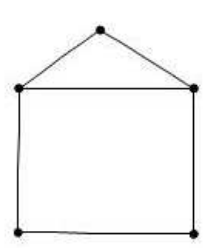

$\mathrm{H}_{5}$

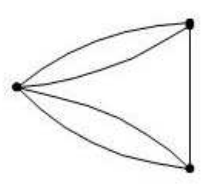

$\mathrm{H}_{8}$

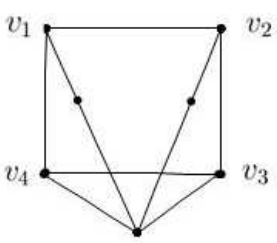

$\mathrm{H}_{11}$

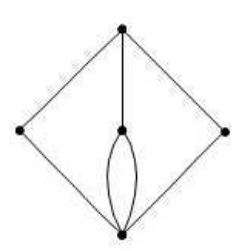

$\mathrm{H}_{6}$

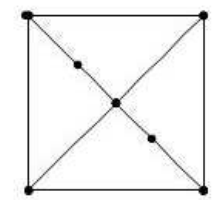

$\mathrm{H}_{9}$

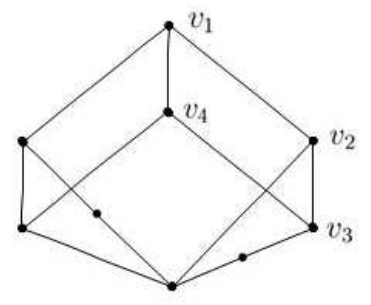

$\mathrm{H}_{12}$

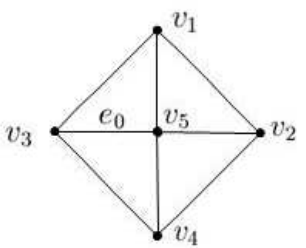

$\mathrm{H}_{7}$

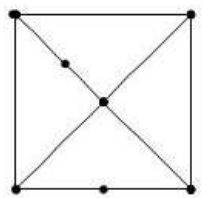

$\mathrm{H}_{10}$

Figure 4.2.5 
Lemma 4.2.13 Let $H_{i}(5 \leq i \leq 12)$ be a graph depicted as Fig 4.2.5. Each of the following hodls.

(i) Both $\mathrm{H}_{5}$ and $\mathrm{H}_{6}$ are collapsible.

(ii) For $\forall X \subseteq E\left(H_{7}\right)$ with $|X|=2$ and $e_{0} \notin X, H_{7}(X)$ is collapsible.

(iii) For $\forall X \subseteq E\left(H_{8}\right)$ with $|X|=2, H_{8}(X)$ is collapsible.

(iv) Both $H_{9}$ and $H_{10}$ are collapsible.

(v) Both $H_{11}$ and $H_{12}$ are collapsible.

Proof (i) Note that $H_{5}$ contains a $C_{3}$ and $\left(H_{5} / C_{3}\right) \cong C_{3}$. Then by Theorem 1.2.1 (i) and (ii), $H_{5}$ is collapsible. Note that $H_{6}$ contains a $C_{2}$ and $H_{6} / C_{2}$ is collapsible by Theorem 1.2.4(ii). Then by Theorem 1.2.1 (i) and (ii), $H_{6}$ is collapsible.

(ii) Suppose there exists $X \subseteq E\left(H_{7}\right)$ with $|X|=2$ and $e_{0} \notin X$ such that $H_{7}(X)$ is not collapsible. Denote $G_{1}$ and $G_{2}$ to be the induced subgraph of $\left\{v_{1}, v_{3}, v_{5}, v_{4}\right\}$ and $\left\{v_{1}, v_{2}, v_{5}, v_{4}\right\}$, respectively. If $\left|X \cap E\left(G_{1}\right)\right| \leq 1$, then $G_{1}(X)$ is isomorphic to a contraction of $H_{5}$, which is collapsible. Then $G_{1}(X)$ is collapsible. Note that $F\left(H_{7}(X) / G_{1}(X)\right) \leq 1$ and it can not be contracted to a $K_{2}$. Thus by Theorem 1.2.4, $H_{7}(X) / G_{1}(X)$ is collapsible, and by Theorem 1.2.1 (i), $H_{7}(X)$ is collapsible, a contradiction. So $X \subseteq E\left(G_{1}\right)$. If $\left|X \cap\left\{v_{1} v_{5}, v_{4} v_{5}\right\}\right| \leq 1$, then $G_{2}(X)$ is isomorphic to a contraction of $H_{5}$ and by a similar argument, we could claim that $H_{7}(X)$ is collapsible, a contradiction. Thus $X=\left\{v_{1} v_{5}, v_{4} v_{5}\right\}$. Then by Lemma $4.2 .12, H_{7}(X)$ is collapsible, a contradiction.

(iii) For $\forall X \subseteq E\left(H_{8}\right)$ with $|X|=2, F\left(H_{8}(X)\right)=0$. Thus by Theorem 1.2.4(ii), $H_{8}(X)$ is collapsible.

(iv) can be viewed as special cases of (ii).

(v) Denote $C$ to be the 4-circuit $v_{1} v_{2} v_{3} v_{4}$ in $H_{11}$. By (iii), $H_{11} / \pi(C)$ is collapsible, and by Theorem 1.2.6 (i), $H_{11}$ is collapsible. Denote $C^{\prime}$ to be the 4-circuit $v_{1} v_{2} v_{3} v_{4}$ in $H_{12}$. Since $H_{12} / \pi\left(C^{\prime}\right) \cong H_{11}$ is collapsible, by Theorem 1.2.6 (i), $H_{12}$ is collapsible.

Lemma 4.2.14 Let $H_{i}(i=13,14,15)$ be a graph depicted as Fig 4.2.6. Then $H_{i}$ is collapsible. 


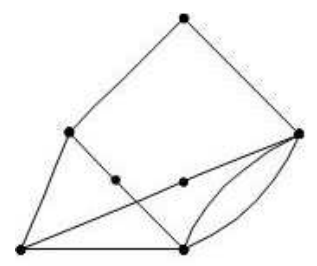

$\mathrm{H}_{13}$

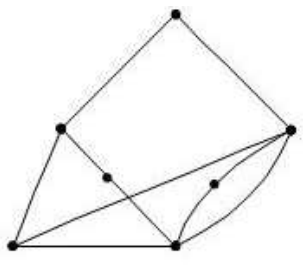

$\mathrm{H}_{14}$

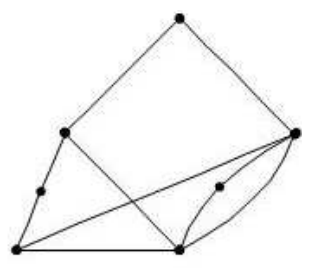

$\mathrm{H}_{15}$

Figure 4.2.6

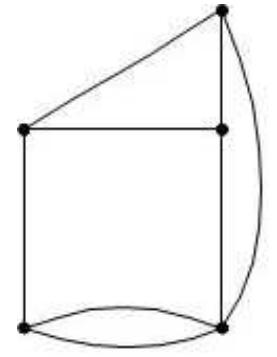

$\mathrm{H}_{16}$

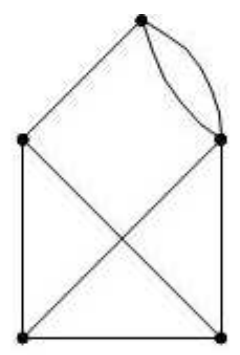

$\mathrm{H}_{17}$

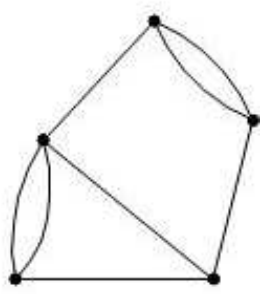

$\mathrm{H}_{18}$

Figure 4.2.7

Proof Note that $F\left(H_{i}\right)=2$ for $i=13,14,15$. By Theorem 1.2.4 (iii), $H_{i}$ is collapsible.

Lemma 4.2.15 Let $H_{i}(i=16,17,18)$ be a graph depicted as Fig 4.2.7. Then $H_{i}$ is collapsible.

Proof Note that $F\left(H_{i}\right)=0$ for $i=16,17,18$. By Theorem 1.2.4 (ii), $H_{i}$ is collapsible.

Lemma 4.2.16 Let $H_{i}(19 \leq i \leq 22)$ be a graph depicted as Fig 4.2.8. Each of the following hodls.

(i) For $\forall X \subseteq E\left(H_{19}\right)$ with $|X|=2,\left|X \cap\left\{e_{1}, e_{2}, e_{3}, e_{4}\right\}\right|=1$ and $\left|X \cap\left\{e_{5}, e_{6}\right\}\right|=1$, 


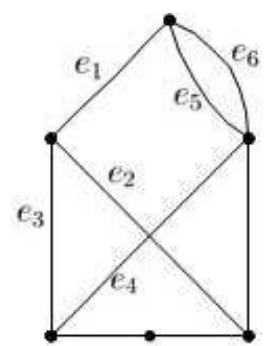

$\mathrm{H}_{19}$

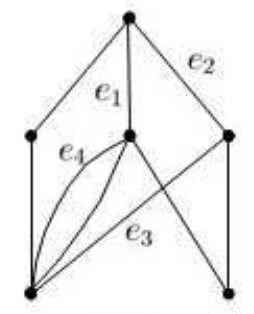

$\mathrm{H}_{21}$

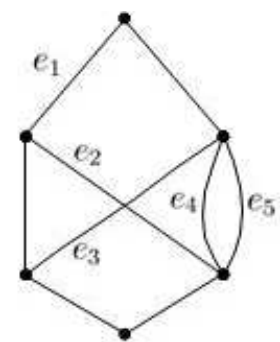

$\mathrm{H}_{20}$

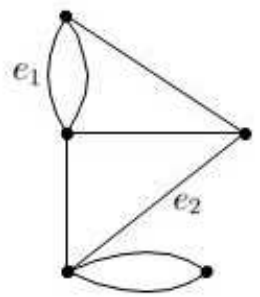

$\mathrm{H}_{22}$

Figure 4.2.8

$H_{19}(X)$ is collapsible.

(ii) For $\forall X \subseteq E\left(H_{20}\right)$ with $|X|=2,\left|X \cap\left\{e_{1}, e_{2}, e_{3}\right\}\right|=1$ and $\left|X \cap\left\{e_{4}, e_{5}\right\}\right|=1, H_{20}(X)$ is collapsible.

(iii) For $\forall X \subseteq E\left(H_{21}\right)$ with $|X|=2,\left|X \cap\left\{e_{1}, e_{2}, e_{3}\right\}\right|=1$ and $e_{4} \in X, H_{21}(X)$ is collapsible.

(iv) $H_{22}\left(e_{1}, e_{2}\right)$ is collapsible.

Proof (i) Without loss of generality, we may assume that $e_{4} \in X$. Note that $e_{4}$ and $e_{5}$ form a 2-circuit in $H_{19}$, denoted by $C$. Then $C\left(e_{4}\right)$ is a triangle in $H_{19}(X)$. Since $H_{19}(X) / C\left(e_{4}\right)$ can be viewed as a graph obtained by subdividing two edges of a $K_{4}$, by Lemma $4.2 .11, H_{19}(X) / C\left(e_{4}\right)$ is collapsible. Thus by Theorem 1.2.1 (i), $H_{19}(X)$ is collapsible.

(ii) can be proved similarly. 
(iii) Denoted by $C$ to be the 2-circuit in $H_{21}$ containing $e_{4}$. Then $C\left(e_{4}\right)$ is a triangle in $H_{21}(X)$. Since 3-circuit is collapsible, the reduction of $H_{21}(X) / C\left(e_{4}\right)$ is $K_{1}$, and $H_{21}(X) / C\left(e_{4}\right)$ is collapsible. Thus by Theorem 1.2.1 (i), $H_{21}(X)$ is collapsible.

(iv) $H_{22}\left(e_{1}, e_{2}\right)$ contains a subgraph isomorphic to $H_{5}$ and $H_{22}\left(e_{1}, e_{2}\right) / H_{5}$ is isomorphic to $C_{2}$. Thus by Lemma 4.2.13(i) and Theorem 1.2.1 (i), $H_{22}\left(e_{1}, e_{2}\right)$ is collapsible.

\subsection{Local Graphs of Small Degree Vertices in a P3D Graph}

In this section, we shall investigate the local structure of vertices with degree at most 3 in a $P_{3}$-dominating graph. Such analysis will be useful in the proof of the main result. Throughout this section, $G$ denotes a connected $P_{3}$-dominating graph. To avoid triviality, we assume that $|V(G)| \geq 2$. For $\forall u \in D_{3}(G)$, the local subgraph of $u$, denoted by $L(u)$, is a subgraph of $G$ containing $E_{G}(u)$.

Lemma 4.3.1 Let $G$ be an essential 4-edge-connected P3D graph and $u \in D_{3}(G)$ with $N_{G}(u)=\left\{a_{1}, a_{2}, a_{3}\right\}$. If $N_{G}(u)$ is an independent set, then $L(u)$ must be one member of $\left\{L_{1}, L_{2}, L_{3}, L_{4}\right\}$, which are depicted as Fig 4.3.1.

Proof Let $u \in D_{3}(G)$ be given. Suppose $N_{G}(u)=\left\{a_{1}, a_{2}, a_{3}\right\}$. There are two possible cases: (i) there exists $i, j \in\{1,2,3\}$ such that $J\left(a_{i}, a_{j}\right)=\emptyset$; (ii) or for $\forall i, j \in\{1,2,3\}$, $J\left(a_{i}, a_{j}\right) \neq \emptyset$

Case $1 J\left(a_{i}, a_{j}\right)=\emptyset$ for some $i, j \in\{1,2,3\}$

Without loss of generality, we could assume that $J\left(a_{1}, a_{3}\right)=\emptyset$.

Claim $1 u \in J^{\prime}\left(a_{1}, a_{3}\right)$.

Suppose $u \notin J^{\prime}\left(a_{1}, a_{3}\right)$. Since $G$ is a P3D graph and $\operatorname{dist}_{G}\left(a_{1}, a_{3}\right)=2$, choose $x \in$ $J^{\prime}\left(a_{1}, a_{3}\right)$. Then by the definition of $J^{\prime}\left(a_{1}, a_{3}\right)$, there exists $y \in N_{G}(x)-\left(N_{G}\left(a_{1}\right) \cup N_{G}\left(a_{3}\right)\right)$ 

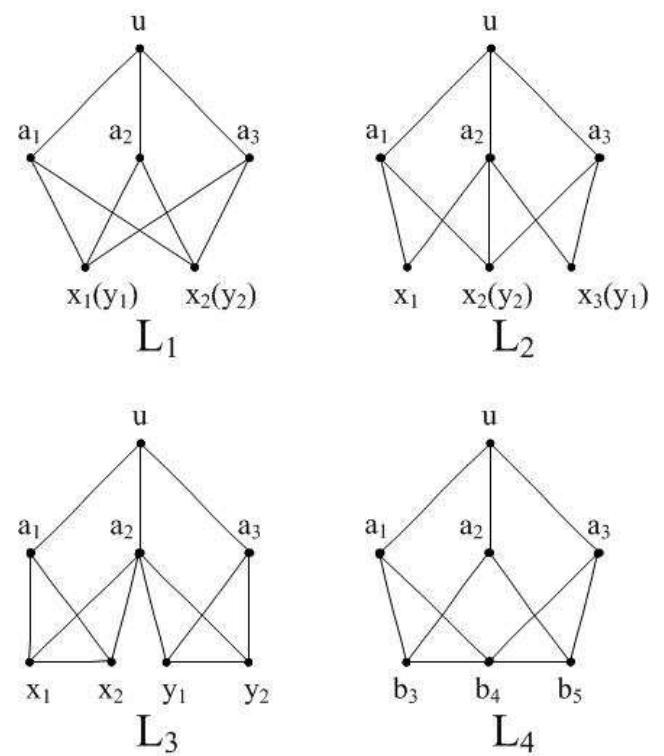

Figure 4.3.1

such that $N_{G}\left(a_{1}\right) \cup N_{G}\left(a_{3}\right) \cup N_{G}(x)-\left\{a_{1}, a_{3}, y\right\} \subseteq N_{G}(y)$. Therefore $u \in N_{G}(y)$, and then $y=a_{2}$. Thus $N_{G}\left(a_{1}\right) \cup N_{G}\left(a_{3}\right)-\left\{a_{1}, a_{2}, a_{3}\right\} \subseteq N_{G}\left(a_{2}\right)$, and $u \in J^{\prime}\left(a_{1}, a_{3}\right)$, which contradicts to $u \notin J^{\prime}\left(a_{1}, a_{3}\right)$. This verifies Claim 1 .

Since $G$ is essentially 4 -edge-connected, $d_{G}\left(a_{i}\right) \geq 2(i=1,2,3)$. Choose $x_{1}, x_{2} \in$ $N_{G}\left(a_{1}\right)-u$ and $y_{1}, y_{2} \in N_{G}\left(a_{3}\right)-u$ such that $\left|\left\{x_{1}, x_{2}\right\} \cap\left\{y_{1}, y_{2}\right\}\right|$ as big as possible. Then $X=\left\{x_{1}, x_{2}, y_{1}, y_{2}\right\}$ is a collection of vertices of $G$.

Subcase $1.1|X|=2$ (that is $\left|\left\{x_{1}, x_{2}\right\} \cap\left\{y_{1}, y_{2}\right\}\right|=2$ )

Then $\left\{x_{1}, x_{2}\right\}=\left\{y_{1}, y_{2}\right\}$ and $L(u)=L_{1}$.

Subcase $1.2|X|=3$ (that is $\left|\left\{x_{1}, x_{2}\right\} \cap\left\{y_{1}, y_{2}\right\}\right|=1$ )

Assume that $\left\{x_{1}, x_{2}\right\} \cap\left\{y_{1}, y_{2}\right\}=x_{2}$. Then $L(u)=L_{2}$.

Subcase $1.3|X|=4$ (that is $\left\{x_{1}, x_{2}\right\} \cap\left\{y_{1}, y_{2}\right\}=\emptyset$ )

Claim $2 \quad x_{1} x_{2} \in E(G)$ and $y_{1} y_{2} \in E(G)$. 
Note that, by the choice of $X, x_{1}, x_{2} \notin N_{G}\left(a_{3}\right)$. It is sufficient to show that $x_{1} x_{2} \in$ $E(G)$. By way of contradiction, suppose $x_{1} x_{2} \notin E(G)$. Since $\operatorname{dist}_{G}\left(u, x_{1}\right)=2, J\left(u, x_{1}\right) \cup$ $J^{\prime}\left(u, x_{1}\right) \neq \emptyset$, and $J\left(u, x_{1}\right) \cup J^{\prime}\left(u, x_{1}\right) \subseteq N_{G}(u) \cap N_{G}\left(x_{1}\right)=\left\{a_{1}, a_{2}\right\}$. Assume that $a_{i} \in J\left(u, x_{1}\right) \cup J^{\prime}\left(u, x_{1}\right)$ for some $i \in\{1,2\}$. Since $x_{2} \notin N_{G}(u) \cup N_{G}\left(x_{1}\right), a_{i} \notin J\left(u, x_{1}\right)$. Thus $a_{i} \in J^{\prime}\left(u, x_{1}\right)$ and $N_{G}(u) \cup N_{G}\left(x_{1}\right) \cup N_{G}\left(a_{i}\right)-\left\{u, x_{1}, a_{i}\right\} \subseteq N_{G}\left(x_{2}\right)$. Therefore $x_{2} a_{3} \in E(G)$, which contradicts to $x_{2} \notin N_{G}\left(a_{3}\right)$. This verifies Claim 2.

By Claim 2, when $\left\{x_{1}, x_{2}\right\} \cap\left\{y_{1}, y_{2}\right\}=\emptyset, L(u)=L_{3}$.

Case 2 for $\forall i, j \in\{1,2,3\}, J\left(a_{i}, a_{j}\right) \neq \emptyset$

Claim $3 J\left(a_{i}, a_{j}\right) \cap J\left(a_{i}, a_{k}\right)=\emptyset$ where $\{i, j, k\}=\{1,2,3\}$.

By way of contradiction, we could assume that $x \in J\left(a_{1}, a_{2}\right) \cap J\left(a_{1}, a_{3}\right)$. Since $x \in$ $J\left(a_{1}, a_{2}\right), N_{G}(x) \subseteq N_{G}\left(a_{1}\right) \cup N_{G}\left(a_{2}\right)$. Since $x \in J\left(a_{1}, a_{3}\right), x a_{3} \in E(G)$. Thus $a_{3} \in$ $N_{G}\left(a_{1}\right) \cup N_{G}\left(a_{2}\right)$, which contradicts to $N_{G}(u)$ is an independent set. This verifies Claim 3 .

By Claim 3, choose $b_{3}, b_{4}$ and $b_{5}$ such that $b_{i+j} \in J\left(a_{i}, a_{j}\right)$ and $b_{i+j} a_{k} \notin E(G)$ since $a_{k} \notin N_{G}\left(a_{i}\right) \cup N_{G}\left(a_{j}\right)(\{i, j, k\}=\{1,2,3\})$.

Claim $4 G\left[\left\{b_{3}, b_{4}, b_{5}\right\}\right]$ has at least two edges.

By way of contradiction, suppose $G\left[\left\{b_{3}, b_{4}, b_{5}\right\}\right]$ has at most one edge. Assume that $b_{3} b_{4}, b_{3} b_{5} \notin E(G)$. Since $\operatorname{dist}_{G}\left(u, b_{3}\right)=2, J\left(u, b_{3}\right) \cup J^{\prime}\left(u, b_{3}\right) \neq \emptyset$, and $J\left(u, b_{3}\right) \cup J^{\prime}\left(u, b_{3}\right) \subseteq$ $N_{G}(u) \cap N_{G}\left(b_{3}\right)=\left\{a_{1}, a_{2}\right\}$. Assume that $a_{i} \in J\left(u, b_{3}\right) \cup J^{\prime}\left(u, b_{3}\right)$ and $\{i, j\}=\{1,2\}$. Since $b_{i+3} \notin N_{G}(u) \cup N_{G}\left(b_{3}\right), a_{i} \notin J\left(u, b_{3}\right)$. Thus $a_{i} \in J^{\prime}\left(u, b_{3}\right)$, and then $N_{G}(u) \cup$ $N_{G}\left(b_{3}\right) \cup N_{G}\left(a_{i}\right)-\left\{u, b_{3}, a_{i}\right\} \subseteq N_{G}\left(b_{i+3}\right)$. Therefore $b_{i+3} a_{j} \in E(G)$, which contradicts to $b_{4} a_{2}, b_{5} a_{1} \notin E(G)$. This verifies Claim 4 .

By Claim 4, we could assume that $b_{3} b_{4}, b_{4} b_{5} \in E(G)$. Then $L(u)=L_{4}$.

Note 1. In $L_{2}, a_{1} x_{3}, a_{3} x_{1} \notin E(G)$, otherwise, the local graph will be $L_{1}$.

Lemma 4.3.2 Let $H \in\left\{L_{1}, L_{2}, L_{3}, L_{4}\right\}$ and let $X \subseteq E(H)$. Then each of the following holds. 
(i) If $|X| \leq 1$, then $H(X)$ is collapsible.

(ii) If $|X| \leq 2$, then either $H(X)$ is collapsible, or $H=L_{2}$.

Proof By Theorem 1.2.1 (vi), it is sufficient to show that $L_{2}(X)$ is collapsible for $\forall X \subseteq$ $E\left(L_{2}\right)$ with $|X|=1$, and $L_{i}(X)$ is collapsible for $\forall X \subseteq E\left(L_{i}\right)$ with $|X|=2(i=1,3,4)$.

Let $X_{1} \subseteq E\left(L_{1}\right)$ with $\left|X_{1}\right|=2$. Since $L_{1} \cong K_{3,3}$, by symmetry, we can assume that $X_{1}=\left\{x_{1} y_{1}, x_{1} y_{2}\right\}$ or $\left\{x_{1} y_{1}, x_{2} y_{2}\right\}$. Let $C_{1}=\left\{\begin{array}{ll}x_{2} y_{2} x_{3} y_{3}, & \text { if } X_{1}=\left\{x_{1} y_{1}, x_{1} y_{2}\right\} \\ x_{2} y_{1} x_{3} y_{3}, & \text { if } X_{1}=\left\{x_{1} y_{1}, x_{2} y_{2}\right\}\end{array}\right.$ be a 4-circuit of $L_{1}\left(X_{1}\right)$. Then $L_{1}\left(X_{1}\right) / \pi\left(C_{1}\right)$ is isomorphic to either $H_{1}$ or $H_{2}$ of Fig 3.1, which are collapsible by Lemma 4.2.9. Thus, by Theorem 1.2.6 (i), $L_{1}\left(X_{1}\right)$ is collapsible.

Let $X_{2} \subseteq E\left(L_{2}\right)$ with $\left|X_{2}\right|=1$. Suppose $X_{2}=\{e\}$. Note that both $L_{2}-x_{1}$ and $L_{2}-y_{1}$ are isomorphic to $K_{3,3}-e$, which is collapsible by Theorem 1.2.1 (vii). If $e \in E_{L_{2}}\left(x_{1}\right)$, then both $L_{2}-x_{1}$ and $L_{2}(e) /\left(L_{2}-x_{1}\right) \cong C_{3}$ are collapsible. Thus by Theorem 1.2.1 (i), $L_{2}(e)$ is collapsible. By symmetry, $L_{2}(e)$ is collapsible if $e \in E_{L_{2}}\left(y_{1}\right)$. Therefore, $e \in E\left(L_{2}\right) \subseteq\left(E_{L_{2}}\left(x_{1}\right) \cup E_{L_{2}}\left(y_{1}\right)\right)$, and by symmetry, we may assume that $e=u a_{1}$ or $u a_{2}$. Let $C_{2}=a_{1} x_{1} a_{2} x_{2}$ be a 4 -circuit of $L_{2}(e)$. Then $L_{2}(e) / \pi\left(C_{2}\right)$ is isomorphic to $H_{3}$ of Fig 3.2, which are collapsible by Lemma 4.2.10. Thus, by Theorem 1.2.6 (i), $L_{2}(e)$ is collapsible.

Let $X_{3} \subseteq E\left(L_{3}\right)$ with $\left|X_{3}\right|=2$. Denote $V_{1}=\left\{u, a_{1}, a_{2}, x_{1}, x_{2}\right\}$ and $V_{2}=\left\{u, a_{2}, a_{3}, y_{1}, y_{2}\right\}$. Denote that $G_{i}=L_{3}\left[V_{i}\right](i=1,2)$. Denote that $X_{3}^{(i)}=X \cap E\left(G_{i}\right)$ for $i=1,2$. Without loss of generality, we assume that $\left|X_{3}^{(1)}\right| \leq\left|X_{3}^{(2)}\right|$. Then $\left|X_{3}^{(1)}\right| \leq 1$ and $G_{1}\left(X_{3}^{(1)}\right)$ is isomorphic to either $H_{4}$ of Fig 3.4 or $K_{4}\left(e^{\prime}\right)$. By Lemma 4.2.11, Lemma 4.2.12 and Theorem 1.2.1 (vi), $G_{1}\left(X_{3}^{(1)}\right)$ is collapsible. Note that $L_{3}\left(X_{3}\right) / G_{1}\left(X_{3}^{(1)}\right)$ is isomorphic to either $K_{4}\left(e^{\prime}\right)$ or $K_{4}\left(e^{\prime}, e^{\prime \prime}\right)$. By Lemma 4.2 .11 and Theorem 1.2.1 (vi), $L_{3}\left(X_{3}\right) / G_{1}\left(X_{3}^{(1)}\right)$ is also collapsible. Thus by Theorem 1.2.1 (i), $L_{3}\left(X_{3}\right)$ is collapsible.

Let $X_{4} \subseteq E\left(L_{4}\right)$ with $\left|X_{4}\right|=2$. Denote $C_{4}=u a_{1} b_{4} a_{3}$ and $C_{4}^{\prime}=a_{2} b_{3} b_{4} b_{5}$, which are two 4-circuits of $L_{4}$. If $X_{4} \cap E\left(C_{4}\right)=\emptyset$, then $L_{4}\left(X_{4}\right) / \pi\left(C_{4}\right)$ is isomorphic to $H_{7}\left(e^{\prime}, e^{\prime \prime}\right)$ (Fig 4.2.5) where $e_{0} \notin\left\{e^{\prime}, e^{\prime \prime}\right\}$. By Lemma 4.2.13 (ii), $L_{4}\left(X_{4}\right) / \pi\left(C_{4}\right)$ is collapsible, and then by Theorem 1.2.6 (i), $L_{4}\left(X_{4}\right)$ is collapsible. So we may assume that $X_{4} \cap E\left(C_{4}\right) \neq \emptyset$. Similarly, $X_{4} \cap E\left(C_{4}^{\prime}\right) \neq \emptyset$. Denote $G_{4}^{1}=L_{4}\left[\left\{u, a_{1}, a_{2}, b_{3}, b_{4}\right\}\right]$ and $G_{4}^{2}=L_{4}\left[\left\{u, a_{2}, a_{3}, b_{4}, b_{5}\right\}\right]$. Note 


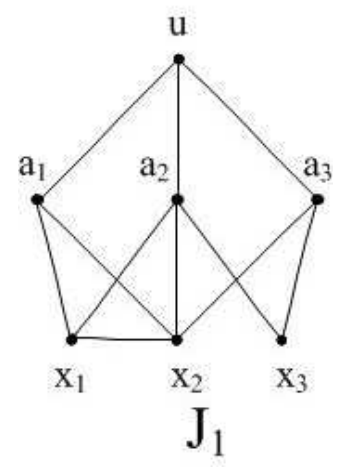

Figure 4.3.2

that $G_{4}^{1} \cong H_{5}$ (Fig 4.2.5), which is collapsible by Lemma 4.2.13 (i). If $X \cap E\left(G_{4}^{1}\right)=\emptyset$, then $L_{4}(X) / G_{4}^{1}$ is isomorphic to $H_{8}\left(e^{\prime}, e^{\prime \prime}\right)$ of Fig 4.2 .5 , which is collapsible by Lemma 4.2.13 (iii). Thus by Theorem 1.2.1 (i), $L_{4}\left(X_{4}\right)$ is collapsible. So we may assume that $X_{4} \cap E\left(G_{4}^{1}\right) \neq \emptyset$. Similarly, $X_{4} \cap E\left(G_{4}^{2}\right) \neq \emptyset$. Hence, by symmetry, we may assume that $\left|X \cap\left\{u a_{1}, a_{1} b_{4}\right\}\right|=\left|X \cap\left\{a_{2} b_{5}, b_{4} b_{5}\right\}\right|=1$.

Suppose $X_{4}=\left\{e^{\prime}, e^{\prime \prime}\right\}$ where $e^{\prime} \in\left\{u a_{1}, a_{1} b_{4}\right\}$ and $e^{\prime \prime} \in\left\{a_{2} b_{5}, b_{4} b_{5}\right\}$. Denote $J_{1}=$ $a_{1} b_{3} b_{4}$ and $J_{2}=a_{3} b_{4} b_{5}$, which are two triangle of $L_{4}$. If $X_{4} \cap J_{1}=\emptyset$, then $L_{4}\left(X_{4}\right) / J_{1}$ is isomorphic to either $H_{9}$ or $H_{10}$ (Fig 4.2.5), which are collapsible by Lemma 4.2.13 (iv). Therefore $X_{4} \cap J_{1} \neq \emptyset$ and then $e^{\prime}=a_{1} b_{4}$. Similarly, $X_{4} \cap J_{2} \neq \emptyset$ and $e^{\prime \prime}=b_{4} b_{5}$. Hence $X_{4}=\left\{a_{1} b_{4}, b_{4} b_{5}\right\}$ and $L_{4}\left(X_{4}\right)$ is isomorphic to $H_{12}$ (Fig 4.2.5), which is collapsible by Lemma $4.2 .13(\mathrm{v})$.

Lemma 4.3.3 Let $J_{1}=L_{2} \cup x_{1} x_{2}$ be the graph depicted in Fig 4.3.2. For any $X \subseteq E\left(J_{1}\right)$ with $|X|=2, J_{1}(X)$ is not reduced.

Proof Suppose $X=\left\{e_{1}, e_{2}\right\}$ is a subset of $E\left(J_{1}\right)$ such that $J_{1}(X)$ is reduced. Since $L_{2} \subseteq J_{1}$, by Lemma 4.3.2 (i), $X \subseteq E\left(L_{2}\right)$ and $x_{1} x_{2} \notin X$. Since $C_{3}=a_{1} x_{1} x_{2}$ is collapsible, $X \cap\left\{a_{1} x_{1}, a_{1} x_{2}\right\} \neq \emptyset$. Since $C_{3}=a_{2} x_{1} x_{2}$ is collapsible, $X \cap\left\{a_{2} x_{1}, a_{2} x_{2}\right\} \neq \emptyset$. Thus we may assume that $e_{1} \in\left\{a_{1} x_{1}, a_{1} x_{2}\right\}$ and $e_{2} \in\left\{a_{2} x_{1}, a_{2} x_{2}\right\}$. Since $J_{1}-x_{1} \cong K_{3,3}-e$, which is collapsible by Theorem 1.2.1 (vii), $X-N_{J_{1}}\left(x_{1}\right) \neq \emptyset$. Thus $X=\left\{a_{1} x_{1}, a_{2} x_{2}\right\}$ 


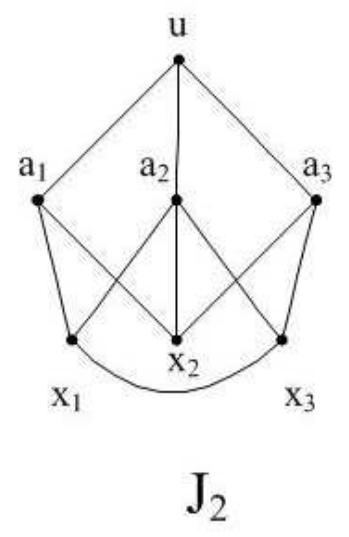

Figure 4.3.3

or $\left\{a_{1} x_{2}, a_{2} x_{1}\right\}$ or $\left\{a_{1} x_{2}, a_{2} x_{2}\right\}$. Denote $C$ to be the 4 -circuit $u a_{2} x_{3} a_{3}$ in $J_{1}(X)$. Since $J_{1}(X) / \pi(C)$ is isomorphic to a member in $\left\{H_{13}, H_{14}, H_{15}\right\}$, by Lemma 4.2 .14 and Theorem 1.2.6 (i), $J_{1}(X)$ is collapsible, a contradiction to the fact that $J_{1}(X)$ is reduced.

Lemma 4.3.4 Let $J_{2}=L_{2} \cup x_{1} x_{3}$ be the graph depicted in Fig 4.3.3. For any $X \subseteq E\left(J_{2}\right)$ with $|X|=2, J_{2}(X)$ is not reduced.

Proof Suppose $X=\left\{e_{1}, e_{2}\right\}$ is a subset of $E\left(J_{2}\right)$ such that $J_{2}(X)$ is reduced.

Since $L_{2} \subseteq J_{2}$, by Lemma 4.3.2 (i), $X \subseteq E\left(L_{2}\right)$ and $x_{1} x_{3} \notin X$. Since $C_{3}=$ $a_{2} x_{1} x_{3}$ is collapsible, $X \cap\left\{a_{2} x_{1}, a_{2} x_{3}\right\} \neq \emptyset$. By symmetry, without loss of generality, we may assume that $e_{1}=a_{2} x_{1}$. Since $J_{2}-x_{1} \cong K_{3,3}-e$, which is collapsible by Theorem 1.2 .1 (vii), $X-N_{J_{2}}\left(x_{1}\right) \neq \emptyset$. Thus $e_{2} \in E\left(J_{2}\right)-N_{J_{2}}\left(x_{1}\right)$. Let $C=$ $\left\{\begin{array}{ll}u a_{2} x_{3} a_{3}, & \text { if } e_{2} \in\left\{u a_{1}, a_{1} x_{2}, a_{2} x_{2}, a_{3} x_{2}\right\} \\ a_{2} x_{2} a_{3} x_{3}, & \text { if } e_{2} \in\left\{u a_{2}, u a_{3}\right\} \\ u a_{1} x_{2} a_{2}, & \text { if } e_{2} \in\left\{a_{2} x_{3}, a_{3} x_{3}\right\}\end{array}\right.$ be a 4-circuit of $J_{2}$ with $E(C) \cap X=\emptyset$. Then $J_{2} / \pi(C)$ is isomorphic to a member of $\left\{H_{16}, H_{17}, H_{18}\right\}$ (Fig 4.2.7), and by Lemma 4.2.15, $F\left(J_{2} / \pi(C)\right)=0$. Thus $F\left(J_{2}(X) / \pi(C)\right) \leq 2$. Note that $J_{2}(X) / \pi(C)$ can not be contracted to $K_{2}$ or a member in $\left\{K_{2, t}: t \geq 1\right\}$. Hence by Theorem 1.2.4 (iii) and Theorem 1.2.6 (i), $J_{2}(X) / \pi(C)$ is collapsible and $J_{2}(X)$ is collapsible, a contradiction to the fact that $J_{2}(X)$ is reduced. 


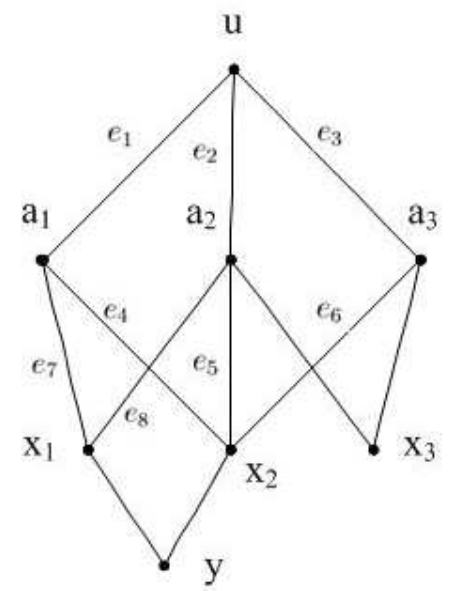

$\mathrm{J}_{3}$

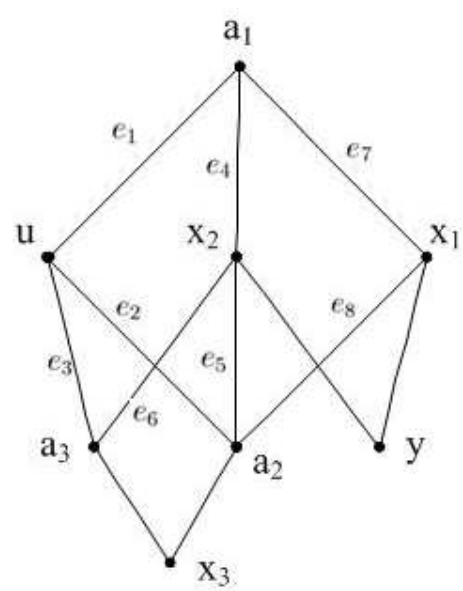

$\mathrm{J}_{3}$

Figure 4.3.4

Lemma 4.3.5 Let $J_{3}$ be the graph depicted in Fig 4.3.4. If $J_{3}(X)$ is reduced for some $X \subseteq E\left(J_{3}\right)$ with $|X|=2$, then $X=\left\{u a_{2}, u a_{3}\right\}$ or $\left\{a_{1} x_{1}, a_{1} x_{2}\right\}$.

Proof Note that $J_{3}$ has an automorphism (Fig 4.3.4). Suppose there exists $X \subseteq E\left(J_{3}\right)$ with $|X|=2$, such that $X$ is neither $\left\{u a_{2}, u a_{3}\right\}$ nor $\left\{a_{1} x_{1}, a_{1} x_{2}\right\}$, and $J_{3}(X)$ is reduced.

Denote $E_{1}=\left\{e_{1}, e_{2}, e_{3}, e_{4}, e_{5}, e_{6}\right\}$ and $E_{2}=\left\{e_{7}, e_{8}\right\}$. Since $L_{2} \subseteq J_{3}$, by Lemma 4.3.2 (i), $X \subseteq E\left(L_{2}\right)$ and $N_{J_{3}}(y) \cap X=\emptyset$. By the automorphism of $J_{3}, N_{J_{3}}\left(x_{3}\right) \cap X=\emptyset$. Thus $X \subseteq E_{1} \cup E_{2}$. Since $J_{3}-\left\{x_{1}, y\right\} \cong K_{3,3}-e$, which is collapsible by Theorem 1.2.1 (vii), $X-E_{2} \neq \emptyset$. So $\left|X \cap E_{1}\right| \geq 1$.

Case $1\left|X \cap E_{1}\right|=2$, which means $X \subseteq E_{1}$.

Since $X \neq E_{2}, X \neq\left\{e_{3}, e_{6}\right\}$ by the automorphism. Also by the automorphism, $J_{3}\left(e_{1}, e_{2}\right) \cong J_{3}\left(e_{1}, e_{4}\right)$ and $J_{3}\left(e_{2}, e_{5}\right) \cong J_{3}\left(e_{4}, e_{5}\right)$. So we may assume that $X \neq\left\{e_{1}, e_{4}\right\}$, $\left\{e_{4}, e_{5}\right\}$. 
Let

$$
C= \begin{cases}a_{2} x_{2} a_{3} x_{3}, & \text { if } X \in\left\{\left\{e_{1}, e_{2}\right\},\left\{e_{1}, e_{3}\right\},\left\{e_{2}, e_{4}\right\},\left\{e_{3}, e_{4}\right\}\right\} \\ u a_{2} x_{3} a_{3}, & \text { if } X \in\left\{\left\{e_{1}, e_{5}\right\},\left\{e_{1}, e_{6}\right\},\left\{e_{4}, e_{6}\right\}\right\} \\ a_{1} x_{1} y x_{2}, & \text { if } X \in\left\{\left\{e_{2}, e_{5}\right\},\left\{e_{3}, e_{5}\right\},\left\{e_{5}, e_{6}\right\}\right\} \\ a_{1} x_{1} a_{2} x_{2}, & \text { if } X=\left\{e_{2}, e_{6}\right\}\end{cases}
$$

be a 4-circuit of $J_{3}$ with $E(C) \cap X=\emptyset$. Then $J_{3} / \pi(C)$ is isomorphic to a member of $\left\{H_{19}, H_{20}, H_{21}, H_{22}\right\}$ (Fig 4.2.8). By Lemma 4.2.16 (i)-(iv), $J_{3}(X) / \pi(C)$ is collapsible. Hence by Theorem 1.2.6 (i), $J_{3}(X)$ is collapsible, a contradiction to the fact that $J_{3}(X)$ is reduced.

Case $2\left|X \cap E_{1}\right|=1$.

In this case $\left|X \cap E_{1}\right|=1$ and $\left|X \cap E_{2}\right|=1$. If $\left(X \cap E_{1}\right) \subseteq\left\{e_{1}, e_{2}, e_{4}, e_{5}\right\}$, then by the automorphism, it could be solved similarly by case 1 . So we assume that $\left(X \cap E_{1}\right) \subseteq$ $\left\{e_{3}, e_{6}\right\}$. Also by the automorphism, $J_{3}\left(e_{3}, e_{8}\right) \cong J_{3}\left(e_{6}, e_{7}\right)$. So we may assume that $X \neq\left\{e_{6}, e_{7}\right\}$.

Let

$$
C= \begin{cases}a_{2} x_{2} a_{3} x_{3}, & \text { if } X \in\left\{\left\{e_{3}, e_{7}\right\},\left\{e_{3}, e_{8}\right\}\right\} \\ u a_{2} x_{3} a_{3}, & \text { if } X=\left\{e_{6}, e_{8}\right\}\end{cases}
$$

be a 4-circuit of $J_{3}$ with $E(C) \cap X=\emptyset$. Then $J_{3} / \pi(C)$ is isomorphic to either $\left\{H_{19}\right.$ or $H_{20}$ (Fig 4.2.8). By Lemma 4.2.16 (i) and (ii), $J_{3}(X) / \pi(C)$ is collapsible. Hence by Theorem $1.2 .6(\mathrm{i}), J_{3}(X)$ is collapsible, a contradiction to the fact that $J_{3}(X)$ is reduced.

Lemma 4.3.6 Let $J_{4}$ be the graph depicted in Fig 4.3.5. Then $J_{4}-\left\{v_{1}, v_{2}\right\}=L_{2}$. For $\forall X \subseteq E\left(L_{2}\right)$ with $|X|=2, J_{4}(X)$ is not reduced.

Proof Suppose there exists $X \subseteq E\left(L_{2}\right)$ with $|X|=2$ such that $J_{4}(X)$ is reduced. Since $\left(L_{2}-x_{1}\right) \cong K_{3,3}-e$ is collapsible, $\left|X \cap\left\{a_{1} x_{1}, a_{2} x_{1}\right\}\right| \leq 1$. Let

$$
C= \begin{cases}z y x_{1} a_{2}, & \text { if } a_{1} x_{1} \in X \\ z y x_{1} a_{1}, & \text { if } a_{2} x_{1} \in X \\ z y x_{1} a_{1}, & \text { if }\left\{a_{1} x_{1}, a_{2} x_{1}\right\} \cap X=\emptyset\end{cases}
$$



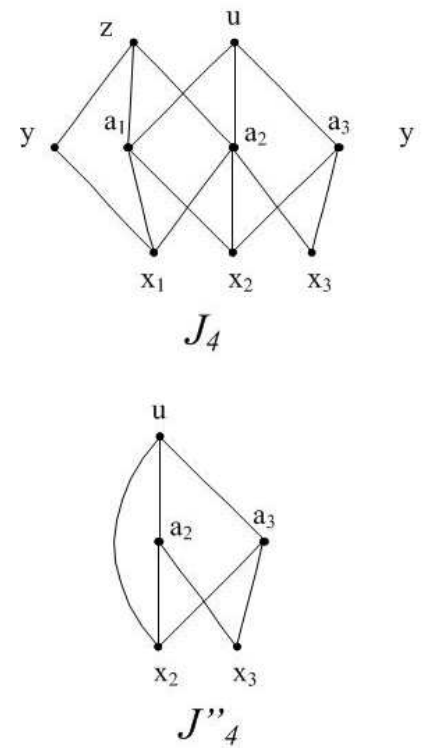
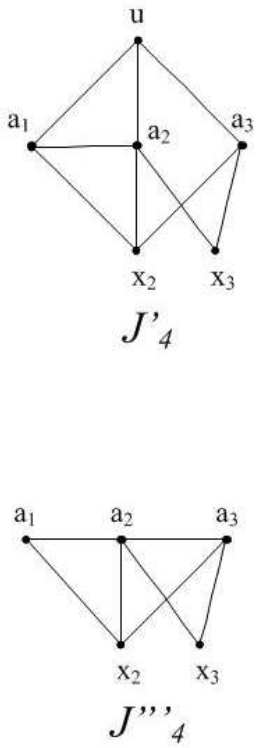

Figure 4.3.5

If $\left|X \cap\left\{a_{1} x_{1}, a_{2} x_{1}\right\}\right|=1$ and assume that $X-\left\{a_{1} x_{1}, a_{2} x_{1}\right\}=e$, then $J_{4}(X) / \pi(C)$ contains a triangle $C_{3}$, and $\left(J_{4}(X) / \pi(C)\right) / C_{3}$ is isomorphic to $J_{4}^{\prime}(e)$, which is depicted in Fig 4.5.3 (b). Note that $J_{4}^{\prime}$ is a contraction of $L_{2}$. By Lemma 4.3.2 (i) and Theorem 1.2.1 (vi), $L_{2}(e)$ is collapsible and $J_{4}^{\prime}(e)$ is collapsible. Thus by Theorem 1.2.1 (i) and Theorem 1.2.6 (i), $J_{4}(X) / \pi(C)$ is collapsible and $J_{4}(X)$ is collapsible, a contradiction.

So we may assume that $\left\{a_{1} x_{1}, a_{2} x_{1}\right\} \cap X=\emptyset$. Let $V_{1}=\left\{z, y, a_{1}, a_{2}, x_{1}, u\right\}$ and $V_{2}=\left\{z, y, a_{1}, a_{2}, x_{1}, x_{2}\right\}$. Since $J_{4}\left[V_{1}\right] \cong K_{3,3}-e, X \cap\left\{u a_{1}, u a_{2}\right\} \neq \emptyset$. Similarly, since $J_{4}\left[V_{2}\right] \cong K_{3,3}-e, X \cap\left\{a_{1} x_{2}, a_{2} x_{2}\right\} \neq \emptyset$. Thus $\left|X \cap\left\{u a_{1}, u a_{2}\right\}\right|=\left|X \cap\left\{a_{1} x_{2}, a_{2} x_{2}\right\}\right|=$ 1. Assume that $e^{\prime}=X \cap\left\{a_{1} x_{2}, a_{2} x_{2}\right\}$. In $J_{4}(X) / \pi(C)$, there is a 2-circuit $C_{2}$, and $\left(J_{4}(X) / \pi(C)\right) / C_{2}$ is isomorphic to $J_{4}^{\prime}(X)$. Note that the triangle $u a_{1} a_{2}$ of $J_{4}^{\prime}(X)$ becomes a 4 -circuit in $J_{4}^{\prime}(X)$, which we may denote by $C^{\prime}$. Then $J_{4}^{\prime}(X) / \pi\left(C^{\prime}\right)$ is isomorphic to either $J_{4}^{\prime \prime}\left(e^{\prime}\right)$ or $J_{4}^{\prime \prime \prime}\left(e^{\prime}\right)$. Since $F\left(J_{4}^{\prime \prime}\right)=F\left(J_{4}^{\prime \prime \prime}\right)=1, F\left(J_{4}^{\prime \prime}\left(e^{\prime}\right)\right)=F\left(J_{4}^{\prime \prime \prime}\left(e^{\prime}\right)\right)=2$. By Theorem 1.2.4 (iii), both $J_{4}^{\prime \prime}\left(e^{\prime}\right)$ and $J_{4}^{\prime \prime \prime}\left(e^{\prime}\right)$ are collapsible. Thus by Theorem 1.2.1 (i) and Theorem 1.2.6 (i), $J_{4}^{\prime}(X) / \pi\left(C^{\prime}\right), J_{4}(X) / \pi(C)$ and $J_{4}(X)$ are collapsible, a contradiction to the fact that $J_{4}(X)$ is reduced. 


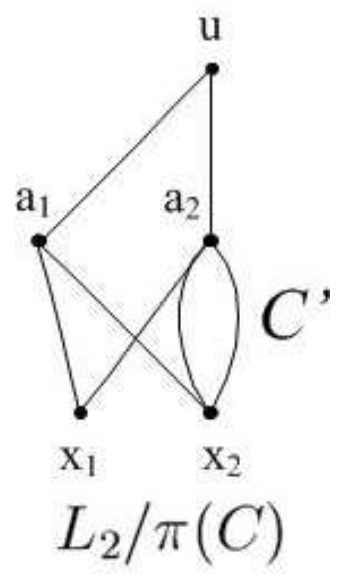

Figure 4.3.6

Lemma 4.3.7 In $L_{2}$, let $X \subseteq E\left(L_{2}\right)-N(u)$ with $|X|=2$. Then each of the following holds.

(i) If $L_{2}(X)$ is reduced, then $X \in\left\{\left\{a_{1} x_{1}, a_{3} x_{3}\right\},\left\{a_{1} x_{1}, a_{2} x_{3}\right\},\left\{a_{1} x_{2}, a_{2} x_{1}\right\},\left\{a_{1} x_{1}, a_{1} x_{2}\right\}\right.$, $\left.\left\{a_{1} x_{2}, a_{2} x_{2}\right\},\left\{a_{1} x_{2}, a_{3} x_{2}\right\},\left\{a_{2} x_{1}, a_{2} x_{3}\right\}\right\}$.

(ii) Let $J_{5}=L_{2} \cup a_{1} y a_{3}$. If $J_{5}(X)$ is reduced, then $X \in\left\{\left\{a_{1} x_{2}, a_{2} x_{2}\right\},\left\{a_{1} x_{2}, a_{3} x_{2}\right\}\right\}$.

(iii) Let $J_{3}=L_{2} \cup x_{1} y x_{2}$ (Fig 4.3.4). If $J_{3}(X)$ is reduced, then $X=\left\{a_{1} x_{1}, a_{1} x_{2}\right\}$.

Proof (i) Considering symmetry of $L_{2}$, by way of contradiction, we may assume that there exists $X \in\left\{\left\{a_{1} x_{1}, a_{2} x_{2}\right\},\left\{a_{1} x_{1}, a_{3} x_{2}\right\},\left\{a_{2} x_{1}, a_{3} x_{2}\right\},\left\{a_{1} x_{1}, a_{2} x_{1}\right\},\left\{a_{2} x_{1}, a_{2} x_{2}\right\}\right\}$ such that $L_{2}(X)$ is not reduced. Let $C$ be the 4-circuit $u a_{2} x_{3} a_{3}$ in $L_{2}$. If $X=\left\{a_{1} x_{1}, a_{2} x_{2}\right\}$, then $\left(L_{2}-x_{1}\right)\left(\cong K_{3,3}-e\right)$ is a collapsible subgraph of $L_{2}(X)$, a contradiction. So we may assume that $X \neq\left\{a_{1} x_{1}, a_{2} x_{2}\right\}$. Then in $L_{2} / \pi(C)$, denote $C^{\prime}$ to be the 2-circuit $a_{2} x_{2}$, which becomes a 3 -circuit $C^{\prime}(X)$ in $L_{2}(X) / \pi(C)$ (Fig 4.3.6). Then $\left(L_{2}(X) / \pi(C)\right) / C^{\prime}(X)$ $\left(\cong H_{5}\right)$ is collapsible by Lemma 4.2 .13 (i). Thus by Theorem 1.2.1 (i) and Theorem 1.2.6 (i), $L_{2}(X) / \pi(C)$ is collapsible and $L_{2}(X)$ is collapsible, a contradiction.

(ii) By way of contradiction, suppose there exists $X \notin\left\{\left\{a_{1} x_{2}, a_{2} x_{2}\right\},\left\{a_{1} x_{2}, a_{3} x_{2}\right\}\right\}$ such that $J_{5}(X)$ is reduced. By (i), $X \in\left\{\left\{a_{1} x_{1}, a_{3} x_{3}\right\},\left\{a_{1} x_{1}, a_{2} x_{3}\right\},\left\{a_{1} x_{2}, a_{2} x_{1}\right\},\left\{a_{1} x_{1}, a_{1} x_{2}\right\}\right.$, 


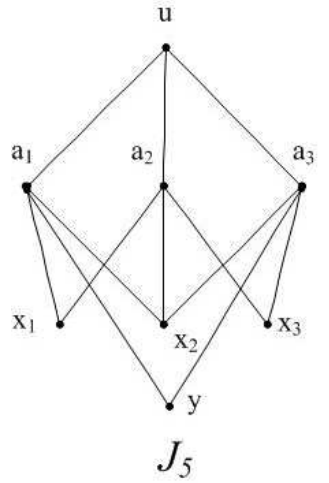

(a)

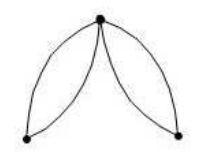

$J{ }_{5}=J_{5} / H$

(d)

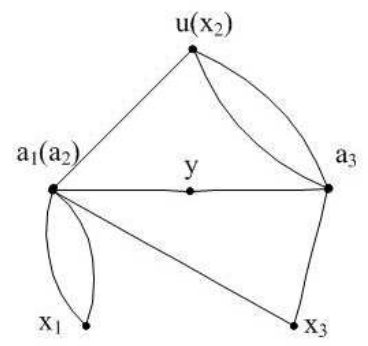

$$
J_{5}^{\prime}=J_{5} / \pi(C)
$$

(b)

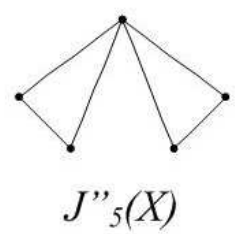

(e)

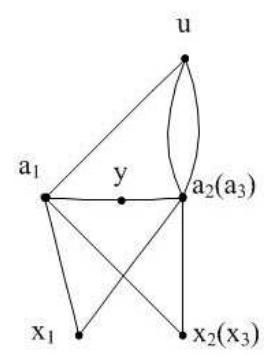

$J_{5}^{\prime}=J_{5} / \pi(C)$

(c)

Figure 4.3.7

$\left.\left\{a_{2} x_{1}, a_{2} x_{3}\right\}\right\}$.

Let

$$
C= \begin{cases}u a_{1} x_{2} a_{2}, & \text { if } X \in\left\{\left\{a_{1} x_{1}, a_{3} x_{3}\right\},\left\{a_{1} x_{1}, a_{2} x_{3}\right\},\left\{a_{2} x_{1}, a_{2} x_{3}\right\}\right\} \\ a_{2} x_{2} a_{3} x_{3}, & \text { if } X \in\left\{\left\{a_{1} x_{2}, a_{2} x_{1}\right\},\left\{a_{1} x_{1}, a_{1} x_{2}\right\}\right\}\end{cases}
$$

Denote $J_{5}^{\prime}=J_{5} / \pi(C)$ which is depicted as Fig 4.3 .7 (b) (if $C=u a_{1} x_{2} a_{2}$ ) or Fig 4.3 .7 (c) (if $C=a_{2} x_{2} a_{3} x_{3}$ ). Note that $E(C) \cap X=\emptyset$, and then $C$ is also a 4-circuit in $J_{5}(X)$. Thus $J_{5}(X) / \pi(C)=J_{5}^{\prime}(X)$ Let

$$
V_{0}= \begin{cases}\left\{u, a_{1}, y, a_{3}\right\}, & \text { if } C=u a_{1} x_{2} a_{2} \\ \left\{u, a_{1}, y, a_{2}\right\}, & \text { if } C=a_{2} x_{2} a_{3} x_{3}\end{cases}
$$

Then the induced subgraph of $V_{0}$ in $J_{5}^{\prime}$, denoted by $H$, is collapsible. Denote $J_{5}^{\prime \prime}=J_{5}^{\prime} / H$, which is depicted as Fig 4.3.7 (d). Note that $X \cap E(H)=\emptyset$. Then $J_{5}^{\prime}(X) / H=J_{5}^{\prime \prime}(X)$, 
which is a collapsible depicted as Fig 4.3.7 (e). Thus by Theorem 1.2.1 (i) and Theorem 1.2.6 (i), $J_{5}^{\prime}(X)$ is collapsible and $J_{5}(X)$ is collapsible, a contradiction.

(iii) By Lemma 4.3.5, $X=\left\{a_{1} x_{1}, a_{1} x_{2}\right\} . \square$

\subsection{Hamiltonian Connectedness of $P_{3}$-dominating Graph- $\mathbf{S}$}

In order to prove Theorem 1.7, we prove the following theorem first.

Theorem 4.4.1 Let $G$ be a 3-edge-connected, essentially 4-edge-connected graph. For $\forall u \in D_{3}(G)$, if $N_{G}(u)$ is an independent set, then $u$ lies in a local subgraph $L(u)$ isomorphic to a contraction of a member in $\left\{L_{1}, L_{2}, L_{3}, L_{4}\right\}$ (Fig 4.4.1). Then for any $X \subseteq E(G)$ with $|X| \leq 2, G(X)$ is collapsible.

Proof For $\forall u \in D_{3}(G)$, if $N_{G}(u)$ is not an independent set, then we assume its local subgraph $L(u)$ is isomorphic to $L_{5}$ (Fig 4.4.1). By contradiction, we assume that

$$
G \text { is a counterexample with }|V(G)| \text { minimized. }
$$

Then for some edge set $X$ with $|X| \leq 2$,

$$
G(X) \text { is not collapsible. }
$$

By Theorem 1.2.1 (vi), we may assume that for $\forall u \in D_{3}(G)$, the local subgraph $L(u)$ is isomorphic to a member in $\left\{L_{1}, L_{2}, L_{3}, L_{4}, L_{5}\right\}$

Claim $1 G(X)$ can not be contracted to a member in $\left\{K_{2}\right\} \cup\left\{K_{2, t}: t \geq 1\right\}$.

Otherwise, it will contradict to the facts that $\kappa^{\prime}(G) \geq 3$ and $D_{2}(G(X)) \leq 2$.

Claim $2 \quad D_{3}(G) \neq \emptyset$. 

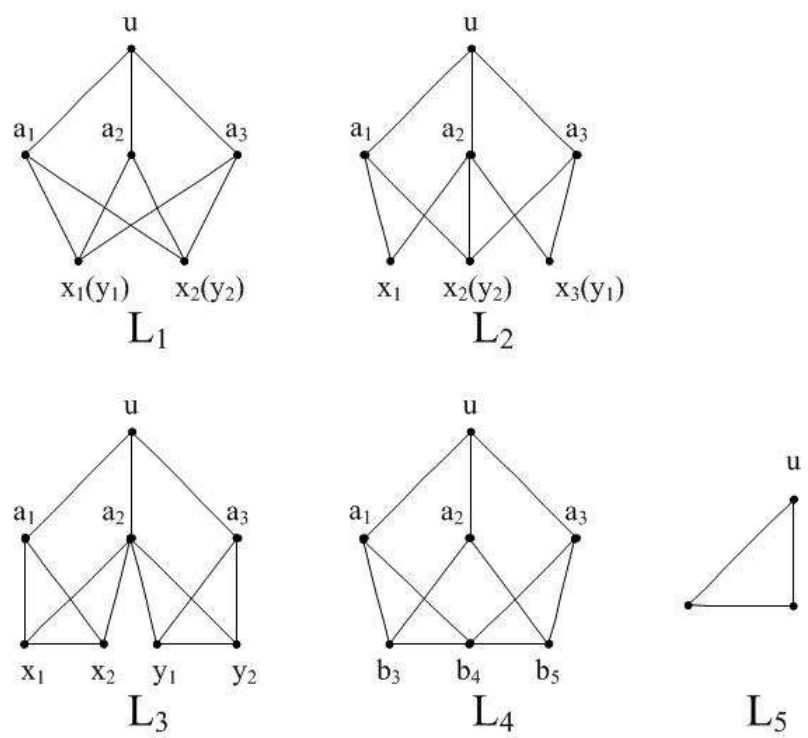

Figure 4.4.1

Suppose $D_{3}(G)=\emptyset$. Since $G$ is 3-edge-connected and essentially 4-edge-connected, every 3-edge-cut of $G$ (if it exists) must be peripheral. Since $D_{3}(G)=\emptyset, G$ is 4-edgeconnected. By Theorem 1.2.2 (i), $G$ has 2 edge-disjoint spanning trees and $F(G)=0$. Then $F(G(X)) \leq 2$. By (4.2) and Theorem 1.2.4 (iii), $G(X)$ can be contracted to a member in $\left\{K_{2}\right\} \cup\left\{K_{2, t}: t \geq 1\right\}$, which contradicts Claim 1. This verifies Claim 2 .

Claim $3 G(X)$ is reduced.

By way of contradiction, let $H$ be a collapsible subgraph of $G(X)$. Denote $X_{1}=$ $X \cap E(H)$ and $X_{2}=X-X_{1}$. Let $H_{0}=H / X_{1}$ and $G^{\prime}=G / H_{0}$. Note that $G^{\prime}$ is a graph satisfying the hypothesis and $\left|V\left(G^{\prime}\right)\right|<|V(G)|$. Thus by (4.1), $G^{\prime}\left(X_{2}\right)$ is collapsible. Since $G^{\prime}\left(X_{2}\right)=G(X) / H$, by Theorem 1.2.1 (i), $G(X)$ is collapsible, a contradiction to (4.2). This verifies Claim 2.

Claim $4\left|D_{3}(G)\right| \geq 6$.

Since $\delta(G) \geq \kappa^{\prime}(G)=3, \delta(G(X)) \geq 2$ and $D_{2}(G(X))=|X| \leq 2$. By Claim 1 and Theorem 1.2.4 (iii), $F(G) \geq 3$. By Claim 3 and Theorem 1.2.4 (i), $F(G(X))=$ 
$2|V(G(X))|-|E(G(X))|-2$. Then

$$
\begin{aligned}
6 & \leq 2 F(G(X))=4|V(G(X))|-2|E(G(X))|-4 \\
& =4(|V(G)|+|X|)-2(|E(G)|+|X|)-4 \\
& =4|V(G)|-2|E(G)|+2|X|-4
\end{aligned}
$$

Since $\delta(G) \geq 3$, we have

$$
2|E(G)| \geq 4\left(\left|V(G)-D_{3}(G)\right|\right)+3\left|D_{3}(G)\right|=4|V(G)|-\left|D_{3}(G)\right| .
$$

Thus, by (4.4) and (4.4)

$$
\left|D_{3}(G)\right| \geq 10-2|X| \geq 10-4=6 .
$$

This verifies Claim 4.

Denote $D_{3}^{\prime}(G)=\left\{u \in D_{3}(G): L(u) \cong L_{i}, 1 \leq i \leq 4\right\}$. By Theorem 4.2.8, $D_{3}^{\prime}(G) \neq \emptyset$. Denote $D_{3}^{\prime \prime}(G)=D_{3}(G)-D_{3}^{\prime}(G)=\left\{u \in D_{3}(G): L(u) \cong L_{5}\right\}$.

Claim $5|X|=2$.

Suppose, by contradiction, that $|X| \leq 1$. Since $D_{3}^{\prime}(G) \neq \emptyset$, choose $u \in D_{3}^{\prime}(G)$, whose local subgraph $L(u)$ is isomorphic to a member in $\left\{L_{1}, L_{2}, L_{3}, L_{4}\right\}$. By Lemma 4.3.2 (i), $G(X)$ is not reduced, which contradicts to Claim 3. This verifies Claim 5.

Claim 6 For $\forall v \in D_{3}^{\prime}(G), L(u) \cong L_{2}$ and $X \subseteq E(L(u))$.

Claim 6 follows by Lemma 4.3.2 and Claim 3.

Claim 7 For $\forall v \in D_{3}^{\prime}(G), L(u)$ is an induced subgraph of $G$.

Choose $u \in D_{3}^{\prime}(G)$, whose local graph $L\left(u_{0}\right) \cong L_{2}$. By Lemma 4.3 .3 and Lemma 4.3.4, $\left\{x_{1}, x_{2}, x_{3}\right\}$ is an independent set. By Note 1 of Lemma 4.3.1, $a_{1} x_{3}, a_{3} x_{1} \notin E(G)$. Thus $L\left(u_{0}\right)$ is a reduced subgraph of $G$. This verifies Claim 7 .

Choose $u_{0} \in D_{3}^{\prime}(G)$, whose local graph $L\left(u_{0}\right) \cong L_{2}$ is shown in Fig 4.4.2 (a).

Claim $8 \quad a_{1}, a_{3} \notin D_{3}(G)$. 


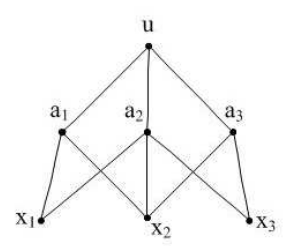

$L\left(u_{0}\right)$

(a)

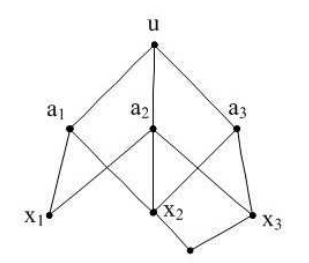

$H^{\prime}=L\left(u_{0}\right) \cup L\left(a_{3}\right)$

(d)

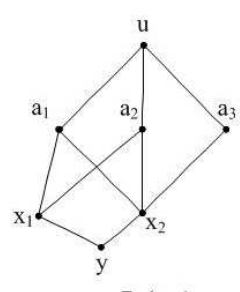

$L\left(a_{1}\right)$

(b)

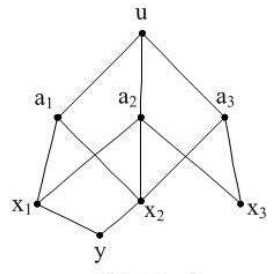

$H \cong J_{3}$

(c)

Figure 4.4.2

By way of contradiction, we assume that $a_{1} \in D_{3}(G)$. Since $N_{G}\left(a_{1}\right)=\left\{u, x_{1}, x_{2}\right\}$ is an independent set, $L\left(a_{1}\right) \cong L_{2}$ (Fig 4.4.2 (b)). Denote $H=L\left(u_{0}\right) \cup L\left(a_{1}\right)$ (Fig 4.4.2 (c)), which is isomorphic to $J_{3}$ (Fig 4.3.5). By Claim 3 and Lemma 4.3.5, $X=\left\{u a_{2}, u a_{3}\right\}$ or $\left\{a_{1} x_{1}, a_{1} x_{2}\right\}$. By the automorphism of $J_{3}, H\left(u a_{2}, u a_{3}\right) \cong H\left(a_{1} x_{1}, a_{1} x_{2}\right)$. Thus without loss of generality, we may assume that $X=\left\{u a_{2}, u a_{3}\right\}$. If $a_{3} \in D_{3}(G)$, then by a similar argument, $L\left(a_{3}\right) \cong L_{2}$ and $H^{\prime}=L\left(u_{0}\right) \cup L\left(a_{3}\right)$ (Fig $4.4 .2(\mathrm{~d})$ ) is isomorphic to $J_{3}$. By Lemma 4.3.5, $H^{\prime}(X)$ is not reduced, a contradiction to Claim 3. Thus we may assume that $a_{3} \notin D_{3}(G)$. Since $\left|D_{3}(G)\right| \geq 6$, choose $v \in D_{3}(G)-\left\{u_{0}, a_{1}\right\}$. Since $v \notin N_{G}\left(u_{0}\right) \cup\left\{x_{2}\right\}, u_{0}$ is not adjacent to $v$. If $L(v) \cong L_{5}$, then $X \cap E(L(v))=\emptyset$, and $L(v)$ is not reduced, which contradicts to Claim 3. So $L(v) \cong L_{2}$. By Claim 6, $X \subseteq E(L(v))$. If $N_{G}(v)=N_{G}\left(u_{0}\right)$, then $\left(L\left(u_{0}\right)-u_{0}\right) \cup G\left[E_{G}(v)\right]\left(\cong L_{2}\right)$ is a collapsible subgraph of $G(X)$, a contradiction. So we may assume that $N_{G}(v) \neq N_{G}\left(u_{0}\right)$. Thus $u_{0}$ is of degree 2 in $L(v)$ (Fig 4.4.2 (e)). Thus $(L(v)-X)\left(\cong K_{3,3}-e\right)$ is a collapsible subgraph of $G(X)$, a contradiction to Claim 3. Hence $a_{1} \notin D_{3}(G)$, and similarly $a_{3} \notin D_{3}(G)$. This verifies Claim 8 .

Claim $9 x_{1}, x_{3} \notin D_{3}^{\prime}(G)$. 
(a)

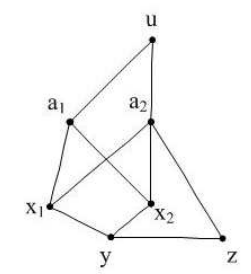

$L\left(x_{1}\right)$

(b)

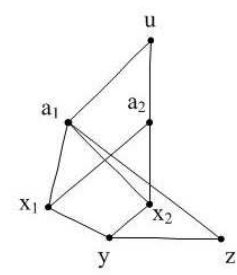

$L\left(x_{1}\right)$

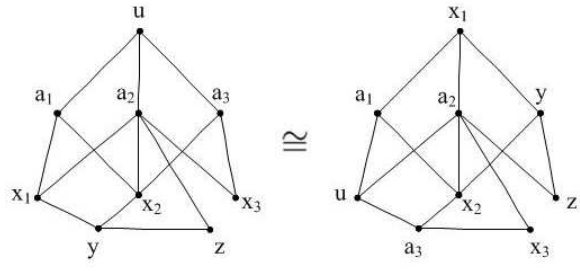

$H$
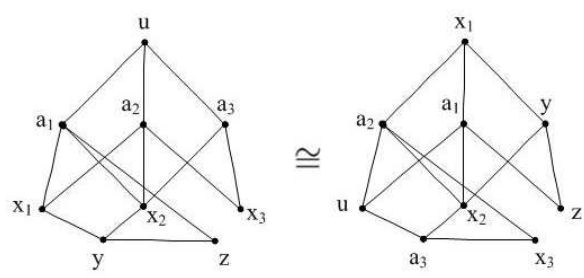

$H$

Figure 4.4.3

By way of contradiction, we assume that $x_{1} \in D_{3}^{\prime}(G)$. Then $L\left(a_{1}\right) \cong L_{2}$, and we may assume that $N_{G}\left(x_{1}\right)=\left\{a_{1}, a_{2}, y\right\}$. If $y x_{2} \notin E(G)$, then by the structure of $L_{2}$, there exists $z \in\left(N_{G}\left(a_{1}\right) \cap N_{G}\left(a_{2}\right) \cap N_{G}(y)-E\left(L\left(u_{0}\right)\right)\right)$, and the graph is isomorphic to $J_{4}$ in Fig 4.3.5. By Lemma 4.3.6, $J_{4}(X)$ is not reduced, a contradiction to Claim 3. Thus $y x_{2} \in E(G)$ and there are two possible cases for $L\left(x_{!}\right)$: Fig 4.4 .3 (a) and (b).

Suppose $L\left(a_{1}\right)$ is shown as Fig 4.4.3 (a). Denote $H=L\left(u_{0}\right) \cup L\left(a_{1}\right)$ (Fig 4.4.3 (a)). Note that $H-z$ is isomorphic to $J_{3}$. By Claim 3 and Lemma 4.3.5, $X=\left\{u a_{2}, u a_{3}\right\}$ or $\left\{a_{1} x_{1}, a_{1} x_{2}\right\}$. Since $\left(L\left(x_{1}\right)-u_{0}\right) \cong K_{3,3}-e$ is a collapsible subgraph in $G, X \cap E\left(\left(L\left(x_{1}\right)-\right.\right.$ $\left.\left.u_{0}\right)\right) \neq \emptyset$. Thus $X=\left\{a_{1} x_{1}, a_{1} x_{2}\right\}$. Note that $H$ has an automorphism (Fig 4.4.3 (a)), and $H-x_{3} \cong J_{3}$. Thus by Lemma 4.3.5, $\left(H-x_{3}\right)(X)$ is not reduced, a contradiction to Claim 3. Similarly, if $L\left(a_{1}\right)$ is shown as Fig 4.4.3 (b), then $X=\left\{a_{1} x_{1}, a_{1} x_{2}\right\}$ and $\left(H-x_{3}\right)(X)$ is not reduced, a contradiction. This verifies Claim 9.

Claim $10 D_{3}^{\prime}(G) \subseteq\left\{u_{0}, x_{2}\right\}$. 


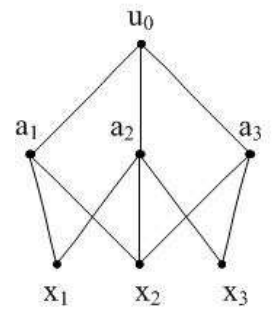

$\mathrm{L}\left(\mathrm{u}_{0}\right)$

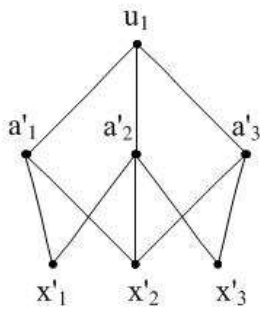

$\mathrm{L}\left(\mathrm{u}_{1}\right)$

Figure 4.4.4

Suppose there exists $u_{1} \in D_{3}^{\prime}(G)-\left\{u_{0}, x_{2}\right\}$ such that $L\left(u_{0}\right) \cong L\left(u_{2}\right) \cong L_{2}$ (Fig 4.4.4). By Claim 8, Claim 9 and $u_{1} \neq x_{2}$,

$$
u_{1} \notin V\left(L\left(u_{0}\right)\right) \text {, and similarly, } u_{0} \notin V\left(L\left(u_{1}\right)\right) \text {. }
$$

Since $u_{1} \neq x_{2}$,

$$
N_{G}\left(u_{0}\right) \neq N_{G}\left(u_{1}\right)
$$

By Claim 8,

$$
u_{1} \notin N_{G}\left(a_{1}\right) \cap N_{G}\left(a_{2}\right) \text {, and similarly, } u_{1} \notin N_{G}\left(a_{2}\right) \cap N_{G}\left(a_{3}\right) \text {. }
$$

By Claim 3, Claim 6 and Lemma 4.3.7 (i), in $L\left(u_{0}\right)$,

$$
\begin{aligned}
X \in & \left\{\left\{a_{1} x_{1}, a_{3} x_{3}\right\},\left\{a_{1} x_{1}, a_{2} x_{3}\right\},\left\{a_{1} x_{2}, a_{2} x_{1}\right\},\left\{a_{1} x_{1}, a_{1} x_{2}\right\}\right. \\
& \left.\left\{a_{1} x_{2}, a_{2} x_{2}\right\},\left\{a_{1} x_{2}, a_{3} x_{2}\right\},\left\{a_{2} x_{1}, a_{2} x_{3}\right\}\right\},
\end{aligned}
$$

and in $L\left(u_{1}\right)$,

$$
\begin{aligned}
X \in\{ & \left\{a_{1}^{\prime} x_{1}^{\prime}, a_{3}^{\prime} x_{3}^{\prime}\right\},\left\{a_{1}^{\prime} x_{1}^{\prime}, a_{2}^{\prime} x_{3}^{\prime}\right\},\left\{a_{1}^{\prime} x_{2}^{\prime}, a_{2}^{\prime} x_{1}^{\prime}\right\},\left\{a_{1}^{\prime} x_{1}^{\prime}, a_{1}^{\prime} x_{2}^{\prime}\right\} \\
& \left.\left\{a_{1}^{\prime} x_{2}^{\prime}, a_{2}^{\prime} x_{2}^{\prime}\right\},\left\{a_{1}^{\prime} x_{2}^{\prime}, a_{3}^{\prime} x_{2}^{\prime}\right\},\left\{a_{2}^{\prime} x_{1}^{\prime}, a_{2}^{\prime} x_{3}^{\prime}\right\}\right\} .
\end{aligned}
$$

Case $1 X=\left\{a_{1} x_{1}, a_{3} x_{3}\right\}$

Since $a_{1} x_{3}, a_{3} x_{1} \notin E(G), X=\left\{a_{1}^{\prime} x_{1}^{\prime}, a_{3}^{\prime} x_{3}^{\prime}\right\}$ in $L\left(u_{1}\right)$. By Lemma 4.3 .7 (ii), $a_{1}$ and $a_{3}$ can not have a common neighbor outside $L\left(u_{0}\right)$. Thus $\left\{a_{1}, a_{3}\right\}=\left\{x_{1}^{\prime}, x_{3}^{\prime}\right\}$. Without 


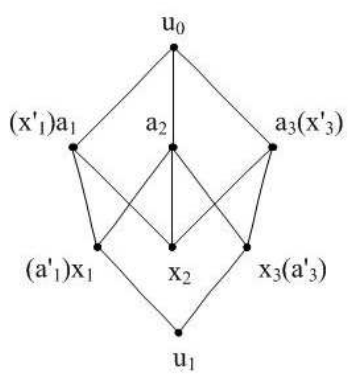

(a)

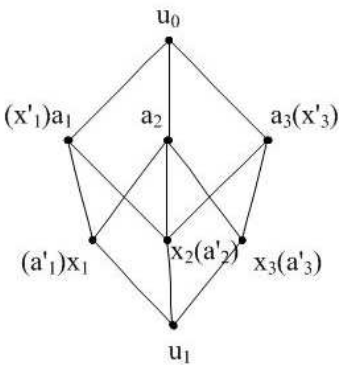

(b)

Figure 4.4.5

loss of generality, we may assume that $a_{1}=x_{1}^{\prime}$ and $a_{3}=x_{3}^{\prime}$. Then $x_{1}=a_{1}^{\prime}$ and $x_{3}=a_{3}^{\prime}$ (Fig 4.4.5 (a)). Since $a_{2}^{\prime}$ is a common neighbor of $x_{1}^{\prime}\left(a_{1}\right)$ and $x_{3}^{\prime}\left(a_{3}\right)$, by Lemma 4.3.7 (ii), $a_{2}^{\prime} \in V\left(L\left(u_{0}\right)\right)$ and then $x_{2}=a_{2}^{\prime}$ (Fig 4.4.5 (b)). By Lemma 4.3 .7 (iii), $\left(L\left(u_{0}\right)+\right.$ $\left.\left\{x_{1} u_{1}, x_{2} u_{1}\right\}\right)(X)$ is not reduced, which contradicts to Claim 3.

Case $2 \quad X=\left\{a_{1} x_{1}, a_{2} x_{3}\right\}$

Since $a_{1} x_{3} \notin E(G)$ and $a_{2} x_{1} \in E(G), X=\left\{a_{1}^{\prime} x_{1}^{\prime}, a_{2}^{\prime} x_{3}^{\prime}\right\}$ in $L\left(u_{1}\right)$. By (4.7), $\left\{a_{1}, a_{2}\right\}=$ $\left\{x_{1}^{\prime}, x_{3}^{\prime}\right\}$, and then $\left\{x_{1}, x_{3}\right\}=\left\{a_{1}^{\prime}, a_{2}^{\prime}\right\}$. Since $a_{1} x_{3} \notin E(G), x_{3}=a_{1}^{\prime}$ and $x_{1}=a_{2}^{\prime}$. And then $a_{1}=x_{3}^{\prime}$ and $a_{2}=x_{1}^{\prime}$. Denote $L=L\left(u_{0}\right)+\left\{x_{1} u_{1}, x_{3} u_{1}\right\}$ and $L(X)$ is depicted as Fig 4.4.6 (a). Denote $C$ to be the 4 -circuit $u_{0} a_{1} x_{2} a_{2}$ in $L(X)$, and denote $L^{\prime}(X)=L(X) / \pi(C)$ (Fig 4.4.6 (b)). Denote $C^{\prime}$ to be the 2-circuit $u_{0} a_{2}$ in $L^{\prime}(X)$, and denote $L^{\prime \prime}(X)=L^{\prime}(X) / C^{\prime}$ (Fig 4.4.6 (c)). Denote $C^{\prime \prime}$ to be the 4-circuit $a_{1} u_{0} v_{a_{2} x_{3}} x_{3}$ in $L^{\prime \prime}(X)$. Since $L^{\prime \prime}(X) / \pi\left(C^{\prime \prime}\right) \cong H_{5}$ (Fig 4.4.6 (b)) is collapsible by Lemma 4.2.13 (i), by Theorem 1.2.1 (i) and Theorem 1.2.6 (i), $L^{\prime \prime}(X), L^{\prime}(X)$ and $L(X)$ are collapsible, a contradiction to Claim 3.

Case $3 \quad X=\left\{a_{1} x_{2}, a_{2} x_{1}\right\}$

Since $a_{1} x_{1}, a_{2} x_{2} \in E(G), X=\left\{a_{1}^{\prime} x_{2}^{\prime}, a_{2}^{\prime} x_{1}^{\prime}\right\}$ in $L\left(u_{1}\right)$. By (4.7), $\left\{a_{1}, a_{2}\right\}=\left\{x_{1}^{\prime}, x_{2}^{\prime}\right\}$, and then $\left\{x_{1}, x_{2}\right\}=\left\{a_{1}^{\prime}, a_{2}^{\prime}\right\}$ (Fig 4.4.7). Let $L=L\left(u_{0}\right)+\left\{x_{1} u_{1}, x_{2} u_{1}\right\}$. By Lemma 4.3.7 (iii), $H(X)$ is not reduced, a contradiction to Claim 3.

Case $4 \quad X=\left\{a_{1} x_{1}, a_{1} x_{2}\right\}$ 


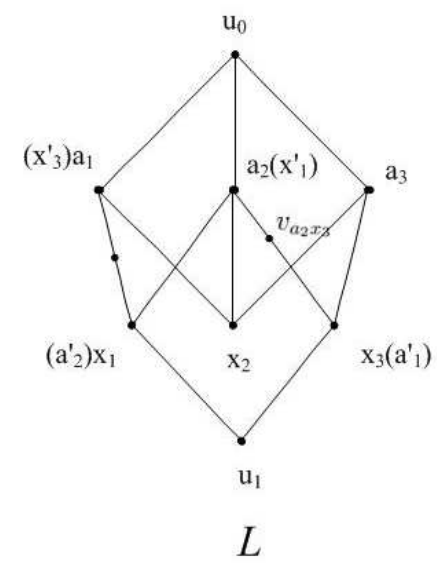

(a)

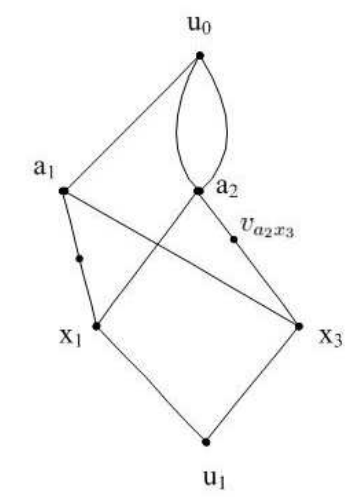

$L^{\prime}(X)=L(X) / \pi(C)$

(b)

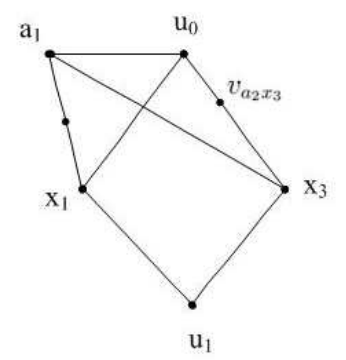

$L^{\prime \prime}(X)=L^{\prime}(X) / C^{\prime}$

(c)

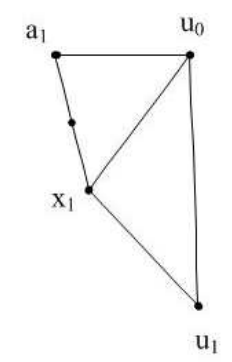

$L^{\prime \prime}(X) / \pi\left(C^{\prime \prime}\right)$

(d)

Figure 4.4.6

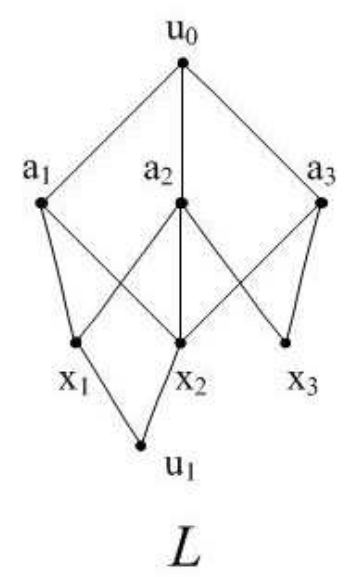

Figure 4.4.7 
(a)

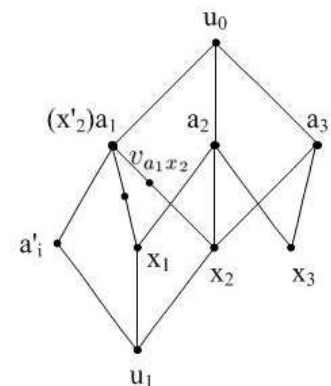

$L(X)$

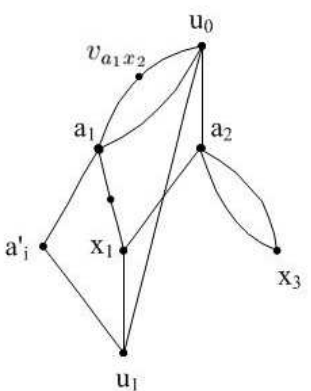

$L^{\prime}(X)=L(X) / \pi(C)$

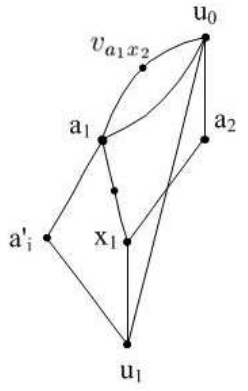

$L^{\prime \prime}(X)=L^{\prime}(X) / C^{\prime}$

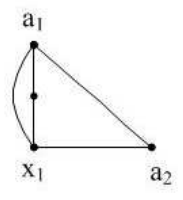

$L^{\prime \prime}(X) / H$

(b)

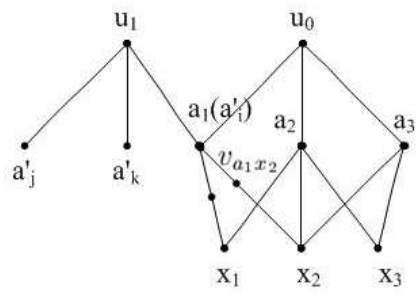

(c)
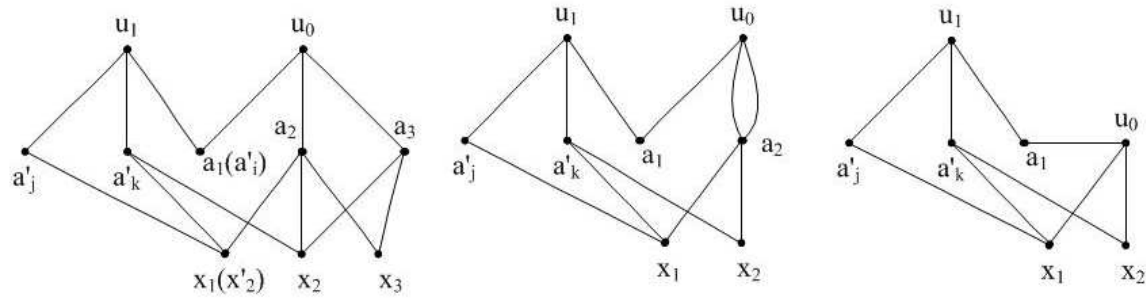

$L$ $L^{\prime}=L / \pi(C)$

$L^{\prime \prime}=L^{\prime} / C^{\prime}$

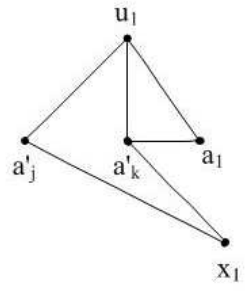

$L^{\prime \prime} / \pi\left(C^{\prime \prime}\right)$

(d)
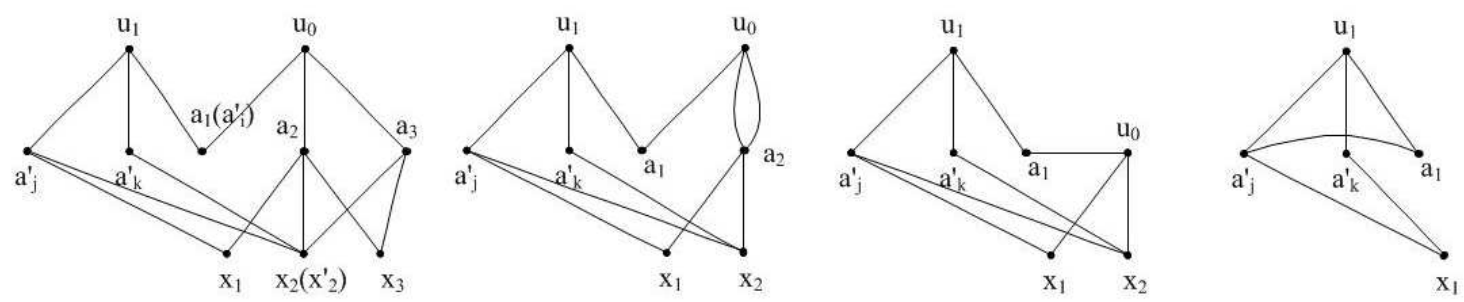

$L$ $L^{\prime}=L / \pi(C)$

$L^{\prime \prime}=L^{\prime} / C^{\prime}$

$L^{\prime \prime} / \pi\left(C^{\prime \prime}\right)$

(e)

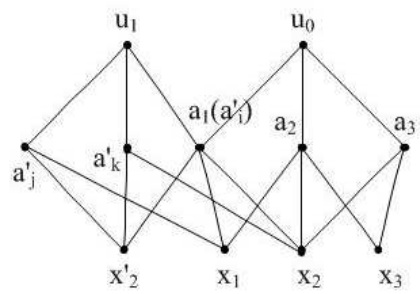

$L\left(u_{0}\right) \cup L\left(u_{1}\right)$

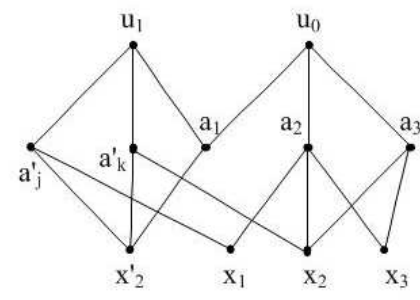

$L$

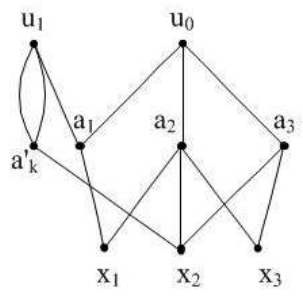

$L^{\prime}=L / \pi(C)$

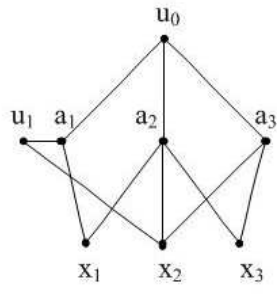

$L^{\prime}=L / \pi(C)$

Figure 4.4 .8 
By the structure of $L_{2}$, either $\left\{x_{1}, x_{2}\right\} \subseteq N_{G}\left(u_{1}\right)$ or $a_{1} \in N_{G}\left(u_{1}\right)$. If $\left\{x_{1}, x_{2}\right\} \subseteq N_{G}\left(u_{1}\right)$, then by the possible cases for $X$ in $L\left(u_{1}\right), a_{1}=x_{2}^{\prime}$. Since $a_{1} x_{3} \neq E(G), x_{3} \neq N_{G}\left(u_{1}\right)$. Assume that $N_{G}\left(u_{1}\right)=\left\{x_{1}, x_{2}, a_{i}^{\prime}\right\}$ and denote $L=L\left(u_{0}\right)+\left\{x_{1} u_{1}, x_{2} u_{i}, a_{i}^{\prime} u_{1}, a_{1} a_{i}^{\prime}\right\} . L(X)$ is depicted as Fig 4.4.8 (a). Denote $C$ to be the 4-circuit $u_{0} a_{2} x_{2} a_{3}$ in $L(X)$, and denote $L^{\prime}(X)=L(X) / \pi(C)$ (Fig 4.4.8 (a)). Denote $C^{\prime}$ to be the 2-circuit $a_{2} x_{3}$ in $L^{\prime}(X)$, and denote $L^{\prime \prime}(X)=L^{\prime}(X) / C^{\prime}$. Denote $H$ to be the induced subgraph of $\left\{u_{0}, a_{1}, v_{a_{1} x_{2}}, a_{i}^{\prime}, u_{1}\right\}$ in $L^{\prime \prime}(X)$. Note that $H \cong H_{5}$ is collapsible by Lemma 4.2 .13 (i). Since 3-circuit is collapsible, the reduction of $L^{\prime \prime}(X) / H$ is $K_{1}$, which means $L^{\prime \prime}(X) / H$ is collapsible. Thus by Theorem 1.2.1 (i) and Theorem 1.2.6 (i), $L^{\prime \prime}(X), L(X)$ and $L(X)$ are all collapsible, a contradiction to Claim 3.

So we may assume that $a_{1} \in N_{G}\left(u_{1}\right)$. By (4.7) and Lemma 4.3.7 (i), $a_{2}, a_{3} \notin N_{G}\left(u_{1}\right)$. We may assume that $a_{1}=a_{i}^{\prime}$, and $N_{G}\left(u_{1}\right)=\left\{a_{i}^{\prime}, a_{j}^{\prime}, a_{k}^{\prime}\right\}$ where $\{i, j, k\}=\{1,2,3\}$ (Fig 4.4.8 (b)). By the structure of $L_{2}, x_{1}, x_{2} \in\left\{x_{1}^{\prime}, x_{2}^{\prime}, x_{3}^{\prime}\right\}$.

If $x_{1}=x_{2}^{\prime}$, then $a_{j}^{\prime} x_{1}, a_{k}^{\prime} x_{1} \in E(G)$. By the structure of $L_{2}, x_{2}$ is adjacent to one of $a_{j}^{\prime}$ and $a_{k}^{\prime}$. Without loss of generality, we may assume that $a_{k}^{\prime} x_{2} \in E(G)$. Denote $L=L\left(u_{0}\right)+\left\{u_{1} a_{1}, u_{1} a_{j}^{\prime}, u_{1} a_{k}^{\prime}, a_{j}^{\prime} x_{1}, a_{k}^{\prime} x_{1}, a_{k}^{\prime} x_{2}\right\}-X$ (Fig 4.4 .8 (c)). Note that $L$ is a subgraph of $G(X)$. Denote $C$ to be the 4-circuit $a_{2} x_{2} a_{3} x_{3}$ in $L$, and denote $L^{\prime}=L / \pi(C)$. Denote $C^{\prime}$ to be the 2-circuit $u_{0} a_{2}$ in $L^{\prime}$, and denote $L^{\prime \prime}=L^{\prime} / C^{\prime}$. Denote $C^{\prime \prime}$ to be the 4 -circuit $u_{0} x_{2} a_{k}^{\prime} x_{1}$ in $L^{\prime \prime}$. Since $L^{\prime \prime} / \pi\left(C^{\prime \prime}\right) \cong H_{5}$, by Lemma 4.2 .13 (i), $L^{\prime \prime} / \pi\left(C^{\prime \prime}\right)$ is collapsible. Thus by Theorem 1.2.1 (i) and Theorem 1.2.6 (i), $L^{\prime \prime}, L^{\prime}$ and $L$ are all collapsible, a contradiction to Claim 3.

If $x_{2}=x_{2}^{\prime}$, then $a_{j}^{\prime} x_{2}, a_{k}^{\prime} x_{2} \in E(G)$. By the structure of $L_{2}, x_{1}$ is adjacent to one of $a_{j}^{\prime}$ and $a_{k}^{\prime}$. Without loss of generality, we may assume that $a_{j}^{\prime} x_{1} \in E(G)$. Denote $L=L\left(u_{0}\right)+\left\{u_{1} a_{1}, u_{1} a_{j}^{\prime}, u_{1} a_{k}^{\prime}, a_{j}^{\prime} x_{2}, a_{k}^{\prime} x_{2}, a_{j}^{\prime} x_{1}\right\}-X($ Fig $4.4 .8(\mathrm{~d}))$. Note that $L$ is a subgraph of $G(X)$. Denote $C$ to be the 4-circuit $a_{2} x_{2} a_{3} x_{3}$ in $L$, and denote $L^{\prime}=L / \pi(C)$. Denote $C^{\prime}$ to be the 2-circuit $u_{0} a_{2}$ in $L^{\prime}$, and denote $L^{\prime \prime}=L^{\prime} / C^{\prime}$. Denote $C^{\prime \prime}$ to be the 4 -circuit $u_{0} x_{2} a_{j}^{\prime} x_{1}$ in $L^{\prime \prime}$. Since $L^{\prime \prime} / \pi\left(C^{\prime \prime}\right) \cong H_{5}$, by Lemma 4.2 .13 (i), $L^{\prime \prime} / \pi\left(C^{\prime \prime}\right)$ is collapsible. Thus by Theorem 1.2.1 (i) and Theorem 1.2.6 (i), $L^{\prime \prime}, L^{\prime}$ and $L$ are all collapsible, a contradiction to Claim 3. 
Thus $\left\{x_{1}, x_{2}\right\}=\left\{x_{1}^{\prime}, x_{3}^{\prime}\right\}$, and then $a_{1}=a_{2}^{\prime}$. By the structure of $L_{2}$, without loss of generality, we may assume that $a_{j}^{\prime} x_{1}, a_{k}^{\prime} x_{2} \in E(G)$. Then $L\left(u_{0}\right) \cup L\left(u_{1}\right)$ is depicted as Fig 4.4.8 (e). Denote $L=L\left(u_{0}\right) \cup L\left(u_{1}\right)-X$. Denote $C$ to be the 4 -circuit $u_{1} a_{1} x_{2}^{\prime} a_{j}^{\prime}$ in $L$, and denote $L^{\prime}=L / \pi(C)$. Denote $C^{\prime}$ to be the 2-circuit $u_{1} a_{k}^{\prime}$ in $L^{\prime}$, and denote $L^{\prime \prime}=L^{\prime} / C^{\prime}$. Since $L^{\prime \prime} / \pi\left(C^{\prime \prime}\right) \cong L\left(u_{0}\right)\left(a_{1} x_{2}\right)$, by Lemma 4.3.2 (i), $L^{\prime \prime} / \pi\left(C^{\prime \prime}\right)$ is collapsible. Thus by Theorem 1.2.1 (i) and Theorem 1.2.6 (i), $L^{\prime \prime}, L^{\prime}$ and $L$ are all collapsible, a contradiction to Claim 3.

Case $5 \quad X=\left\{a_{1} x_{2}, a_{2} x_{2}\right\}$

By (4.7), $\left\{a_{1}, a_{2}\right\} \subseteq\left\{x_{1}^{\prime}, x_{2}^{\prime}, x_{3}^{\prime}\right\}$ and $x_{2} \in N_{G}\left(u_{1}\right)$. By Lemma 4.3 .7 (ii), $x_{1}, x_{3} \notin$ $N_{G}\left(u_{1}\right)$. Assume that $x_{2}=a_{i}^{\prime}$ and $N_{G}\left(u_{1}\right)=\left\{a_{i}^{\prime}, a_{j}^{\prime}, a_{k}^{\prime}\right\}$ where $\{i, j, k\}=\{1,2,3\}$ (Fig 4.4.9 (a)).

If $a_{1}=x_{2}^{\prime}$, then $a_{j}^{\prime} a_{1}, a_{k}^{\prime} a_{1} \in E(G)$. By the structure of $L_{2}, a_{2}$ is adjacent to one of $a_{j}^{\prime}$ and $a_{k}^{\prime}$. Without loss of generality, we may assume that $a_{k}^{\prime} a_{2} \in E(G)$. Denote $L=L\left(u_{0}\right)+\left\{u_{1} x_{2}, u_{1} a_{j}^{\prime}, u_{1} a_{k}^{\prime}, a_{j}^{\prime} a_{1}, a_{k}^{\prime} a_{1}, a_{k}^{\prime} a_{2}\right\}$ and $L(X)$ is depicted as Fig 4.4.9 (b)). Denote $C$ to be the 4 -circuit $u_{0} a_{2} x_{3} a_{2}$ in $L(X)$, and denote $L^{\prime}(X)=L(X) / \pi(C)$. Denote $H$ to be the induced subgraph of $\left\{a_{2}, v_{a_{2} x_{2}}, x_{2}, a_{k}^{\prime}, u_{1}\right\}$ in $L^{\prime}(X)$, and denote $L^{\prime \prime}(X)=$ $L^{\prime} / H$. Denote $C^{\prime}$ to be the 3 -circuit $a_{1} a_{2} v_{a_{1} x_{2}}$ in $L^{\prime \prime}(X)$. Since 2-circuit is collapsible, the reduction of $L^{\prime \prime}(X) / C^{\prime}$ is $K_{1}$, which means $L^{\prime \prime}(X) / C^{\prime}$ is collapsible. Thus by Theorem 1.2.1 (i) and Theorem 1.2.6 (i), $L^{\prime \prime}(X), L^{\prime}(X)$ and $L(X)$ are all collapsible, a contradiction to Claim 3.

If $a_{2}=x_{2}^{\prime}$, then $a_{j}^{\prime} a_{2}, a_{k}^{\prime} a_{2} \in E(G)$. By the structure of $L_{2}, a_{1}$ is adjacent to one of $a_{j}^{\prime}$ and $a_{k}^{\prime}$. Without loss of generality, we may assume that $a_{j}^{\prime} a_{1} \in E(G)$. Denote $L=L\left(u_{0}\right)+\left\{u_{1} x_{2}, u_{1} a_{j}^{\prime}, u_{1} a_{k}^{\prime}, a_{j}^{\prime} a_{2}, a_{k}^{\prime} a_{2}, a_{j}^{\prime} a_{1}\right\}$ (Fig 4.4.9 (c)). Denote $C$ to be the 4circuit $a_{2} a_{j}^{\prime} u_{1} a_{k}^{\prime}$ in $L(X)$, and denote $L^{\prime}(X)=L(X) / \pi(C)$. Denote $C^{\prime}$ to be the 3 -circuit $a_{2} x_{2} v_{a_{2} x_{2}}$ in $L^{\prime}(X)$, and denote $L^{\prime \prime}(X)=L^{\prime} / C^{\prime}$. Denote $H$ to be induced subgraph of $\left\{u_{0}, a_{1}, v_{a_{1} x_{2}}, a_{2}, a_{3}\right\}$ in $L^{\prime \prime}(X)$. Note that $H \cong H_{5}$ is collapsible by Lemma 4.2 .13 (i). Since 2-circuit is collapsible, the reduction of $L^{\prime \prime}(X) / H$ is $K_{1}$, which means $L^{\prime \prime}(X) / H$ is collapsible. Thus by Theorem 1.2.1 (i) and Theorem 1.2.6 (i), $L^{\prime \prime}(X), L^{\prime}(X)$ and $L(X)$ are all collapsible, a contradiction to Claim 3. 
(a)

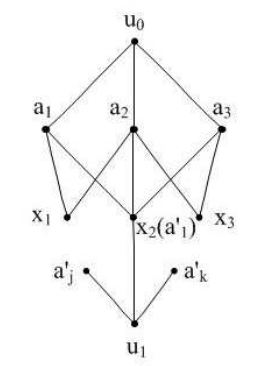

(b)
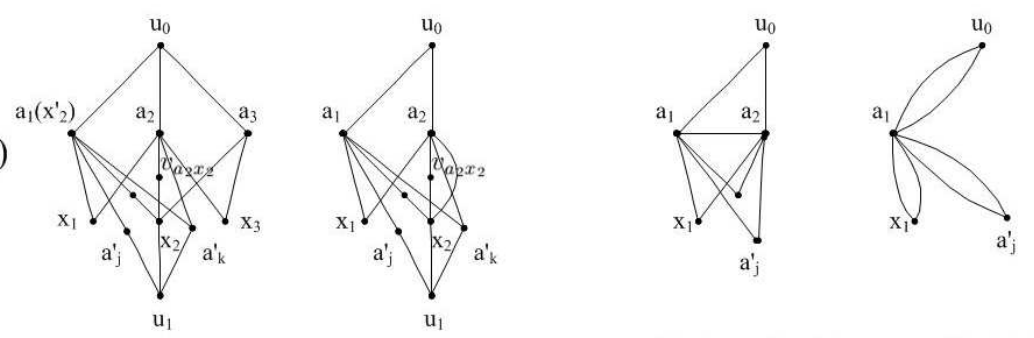

$L(X)$
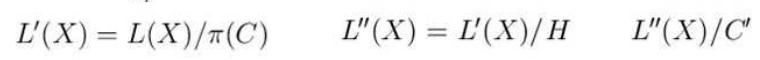

(c)
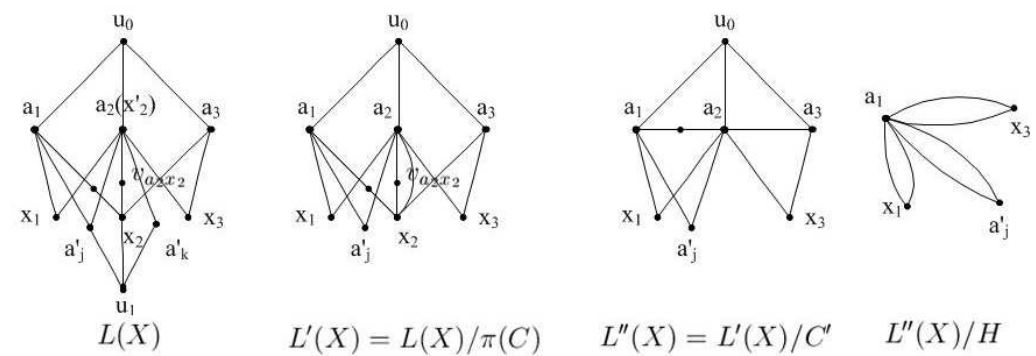

(d)

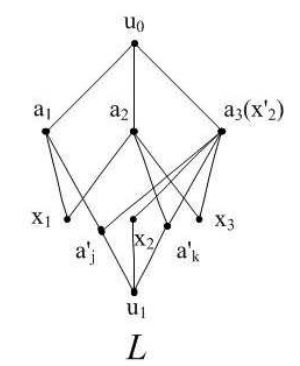

$$
L^{\prime}(X)=L(X) / \pi(C)
$$$$
L^{\prime \prime}(X)=L^{\prime}(X) / C^{\prime} \quad L^{\prime \prime}(X) / H
$$

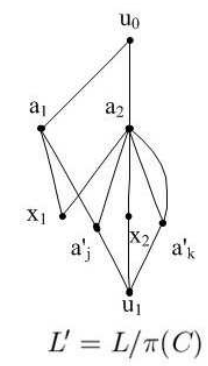

(e)
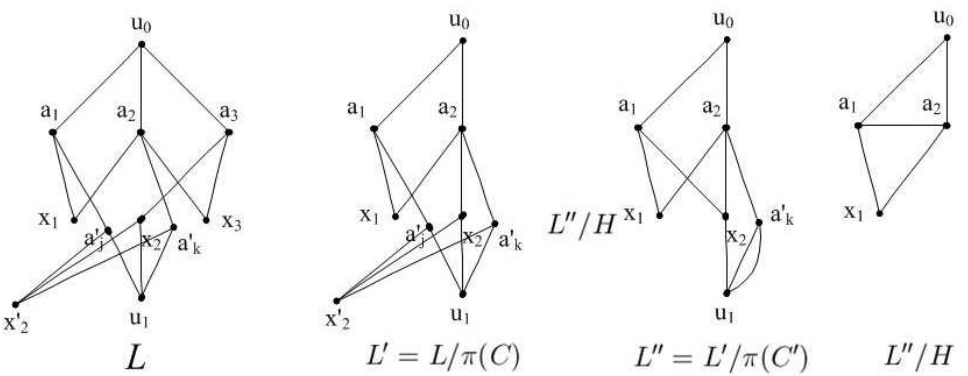

Figure 4.4.9 
Thus $\left\{a_{1}, a_{2}\right\}=\left\{x_{1}^{\prime}, x_{3}^{\prime}\right\}$ and then $x_{2}=a_{2}^{\prime}$. By the structure of $L_{2}$, without loss of generality, we may assume that $a_{j}^{\prime} a_{1}, a_{k}^{\prime} a_{2} \in E(G)$.

If $x_{2}^{\prime}$ is in $L\left(u_{0}\right)$, then $x_{2}^{\prime}=x_{3}$ and $a_{j}^{\prime} a_{3}, a_{k}^{\prime} a_{3} \in E(G)$. Denote $L=L\left(u_{0}\right)+$ $\left\{u_{1} x_{2}, u_{1} a_{j}^{\prime}, u_{1} a_{k}^{\prime}, a_{j}^{\prime} a_{1}, a_{j}^{\prime} a_{3}, a_{k}^{\prime} a_{2}, a_{k}^{\prime} a_{3}\right\}-X$ (Fig 4.4.9 (d)). Note that $L$ is a subgraph of $G(X)$. Denote $C$ to be the 4-circuit $u_{0} a_{2} x_{3} a_{3}$ in $L$, and denote $L^{\prime}=L / \pi(C)$. Since $L^{\prime}$ is a 4-circuit-connected graph containing a 2-circuit, by Lemma $4.2 .1, L^{\prime}$ is collapsible. Thus, by Theorem 1.2.6 (i), $L$ is collapsible, a contradiction to Claim 3.

So we may assume that $x_{2}^{\prime}$ is not in $L\left(u_{0}\right)$. Denote $L=L\left(u_{0}\right) \cup L\left(u_{1}\right)-X$ (Fig 4.4.9 (e)). Denote $C$ to be the 4 -circuit $u_{0} a_{2} x_{3} a_{3}$ in $L$, and denote $L^{\prime}=L / \pi(C)$. Denote $C^{\prime}$ to be the 4 -circuit $a_{j}^{\prime} u_{1} x_{2} x_{2}^{\prime}$ in $L^{\prime}$, and denote $L^{\prime \prime}=L^{\prime} / \pi\left(C^{\prime}\right)$. Denote $H$ to be induced subgraph of $\left\{a_{2}, x_{2}, u_{1}, a_{k}^{\prime}\right\}$ in $L^{\prime \prime}$. Note that $H$ is collapsible by Lemma 4.2.1. Since 3-circuit is collapsible, the reduction of $L^{\prime \prime} / H$ is $K_{1}$, which means $L^{\prime \prime} / H$ is collapsible. Thus by Theorem 1.2.1 (i) and Theorem 1.2.6 (i), $L^{\prime \prime}, L^{\prime}$ and $L$ are all collapsible, a contradiction to Claim 3.

Case $6 \quad X=\left\{a_{1} x_{2}, a_{3} x_{2}\right\}$

We may assume that in $L\left(u_{1}\right), X$ is either $\left\{a_{1}^{\prime} x_{2}^{\prime}, a_{3}^{\prime} x_{2}^{\prime}\right\}$ or $\left\{a_{2}^{\prime} x_{1}^{\prime}, a_{2}^{\prime} x_{3}^{\prime}\right\}$, otherwise we may use a similar argument to previous cases.

If $X=\left\{a_{1}^{\prime} x_{2}^{\prime}, a_{3}^{\prime} x_{2}^{\prime}\right\}$, then $a_{1}=a_{1}^{\prime}, a_{3}=a_{3}^{\prime}$ and $x_{2}=x_{2}^{\prime}$. By (4.6), $a_{2} \neq a_{2}^{\prime}$ and $a_{2}^{\prime}$ is not in $L\left(u_{0}\right)$. By Lemma 4.3 .7 (iii), $x_{1} a_{2}^{\prime}, x_{3} a_{2}^{\prime} \notin E(G)$. Thus $x_{1}^{\prime}$ and $x_{3}^{\prime}$ are not in $L\left(u_{0}\right)$. Denote $L=L\left(u_{0}\right) \cup L\left(u_{1}\right)$ and $L(X)$ is depicted as Fig 4.4.10 (a). Denote $C$ to be the 4 -circuit $u_{0} a_{2} x_{3} a_{3}$ in $L(X)$, and denote $L^{\prime}(X)=L(X) / \pi(C)$. Denote $C^{\prime}$ to be the 3-circuit $a_{2} x_{2} v_{a_{3} x_{2}}$ in $L^{\prime}(X)$, and denote $L^{\prime \prime}(X)=L^{\prime} / C^{\prime}$. Denote $H$ to be induced subgraph of $\left\{u_{1}, a_{1}, v_{a_{1} x_{2}}, a_{2}, a_{2}^{\prime}\right\}$ in $L^{\prime \prime}(X)$. Note that $H \cong H_{5}$ is collapsible by Lemma 4.2.13 (i). Since 2-circuit is collapsible, the reduction of $L^{\prime \prime}(X) / H$ is $K_{1}$, which means $L^{\prime \prime}(X) / H$ is collapsible. Thus by Theorem 1.2.1 (i) and Theorem 1.2.6 (i), $L^{\prime \prime}(X), L^{\prime}(X)$ and $L(X)$ are all collapsible, a contradiction to Claim 3.

Thus we may assume that $X=\left\{a_{2}^{\prime} x_{1}^{\prime}, a_{2}^{\prime} x_{3}^{\prime}\right\}$. Then $a_{1}=x_{1}^{\prime}, a_{3}=x_{3}^{\prime}$ and $x_{2}=a_{2}^{\prime}$. By Lemma 4.3.7 (iii), $x_{1} u_{1}, x_{3} u_{1} \notin E(G)$. Thus $a_{1}^{\prime}$ and $a_{3}^{\prime}$ are not in $L\left(u_{0}\right)$. 
(a)
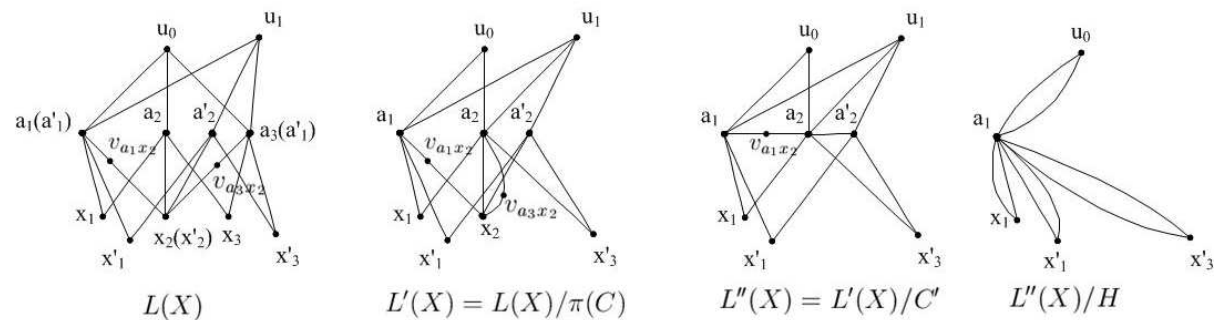

(b)
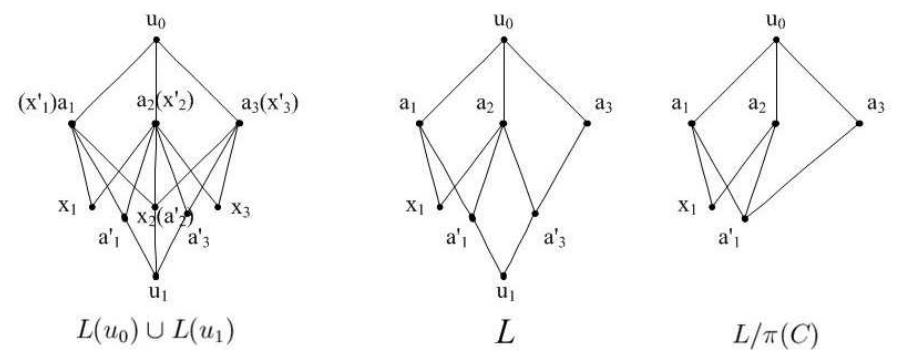

(c)
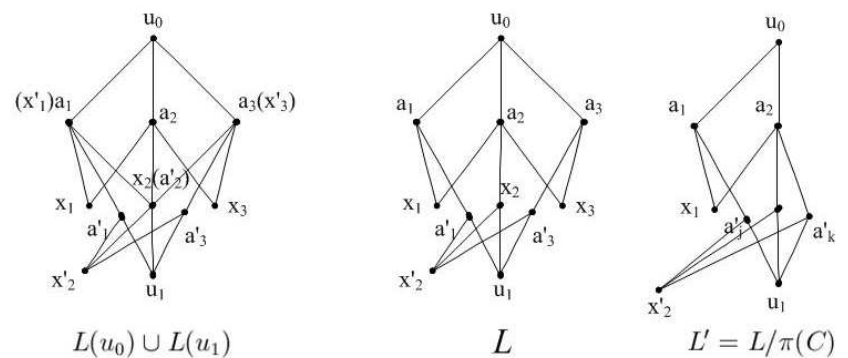

Figure 4.4.10 
If $x_{2}^{\prime}$ is in $L\left(u_{0}\right)$, then $x_{2}^{\prime}=a_{2}$ (Fig 4.4.10 (b)). Denote $L=\left(L\left(u_{0}\right)-\left\{x_{2}, x_{3}\right\}\right)+$ $\left\{a_{1} a_{1}^{\prime}, a_{2} a_{1}^{\prime}, a_{2} a_{3}^{\prime}, a_{3} a_{3}^{\prime}, a_{1}^{\prime} u_{1}, a_{3}^{\prime} u_{1}\right\}$. Note that $L \subseteq G(X)$. Denote $C$ to be the 4 -circuit $a_{2} a_{1}^{\prime} u_{1} a_{3}^{\prime}$ in $L$. Since $L / \pi(C) \cong K_{3,3}-e$ is collapsible, by Theorem 1.2.6 (i), $L$ is collapsible, a contradiction to Claim 3.

So we may assume that $x_{2}^{\prime}$ is not in $L\left(u_{0}\right)$ (Fig 4.4.10 (c)). Denote $L=L\left(u_{0}\right) \cup$ $L\left(u_{1}\right)-X$. Note that $L \subseteq G(X)$. Denote $C$ to be the 4 -circuit $u_{0} a_{2} x_{3} a_{3}$ in $L$. Since $L / \pi(C)$ to $L^{\prime}$ in Fig 4.4 .9 (e), then $L / \pi(C)$ is collapsible. Thus, by Theorem 1.2.6 (i), $L$ is collapsible, a contradiction to Claim 3.

Case $7 \quad X=\left\{a_{2} x_{1}, a_{2} x_{3}\right\}$

We may assume that in $L\left(u_{1}\right), X=\left\{a_{2}^{\prime} x_{1}^{\prime}, a_{2}^{\prime} x_{3}^{\prime}\right\}$, otherwise we may use a similar argument to previous cases. Then $a_{2}=a_{2}^{\prime}, x_{1}=x_{1}^{\prime}$ and $x_{3}=x_{3}^{\prime}$. By (4.7), $a_{1}, a_{3} \notin$ $N_{G}\left(u_{1}\right)$. Thus $a_{1}^{\prime}$ and $a_{3}^{\prime}$ are not in $L\left(u_{0}\right)$. By Lemma 4.3 .7 (iii), $x_{2} a_{1}^{\prime}, x_{2} a_{3}^{\prime} \notin E(G)$. Thus $x_{2}^{\prime}$ is not in $L\left(u_{0}\right)$. Denote $L=L\left(u_{0}\right) \cup L\left(u_{1}\right)$ and $L(X)$ is depicted as Fig 4.4.11. Denote $C$ to be the 4 -circuit $u_{0} a_{2} x_{2} a_{3}$ in $L(X)$ and denote $L^{\prime}(X)=L(X) / \pi(C)$. Denote $C^{\prime}$ to be the 3 -circuit $a_{2} x_{3} v_{a_{2} x_{3}}$ in $L^{\prime}(X)$ and denote $L^{\prime \prime}(X)=L^{\prime}(X) / C^{\prime}$. let $H=$ $L^{\prime \prime}(X)-\left\{u_{0}, a_{1}\right\}$. Note that $H \cong L\left(u_{0}\right)\left(a_{2} x_{1}\right) / a_{2} x_{3}$. By Lemma 4.3 .2 (i) and Theorem 1.2.1 (vi), $L\left(u_{0}\right)\left(a_{2} x_{1}\right) / a_{2} x_{3}$ is collapsible, and then $H$ is collapsible. Since 3-circuit is collapsible, the reduction of $L^{\prime \prime}(X) / H$ is $K_{1}$, which means $L^{\prime \prime}(X) / H$ is collapsible. Thus by Theorem 1.2.1 (i) and Theorem 1.2.6 (i), $L^{\prime \prime}(X), L^{\prime}(X)$ and $L(X)$ are all collapsible, a contradiction to Claim 3.

This verifies Claim 10.

Claim 11 For $\forall v \in D_{3}^{\prime \prime}(G),\left|E(L(v)) \cap E\left(L\left(u_{0}\right)\right)\right|=|E(L(v)) \cap X|=1$. Moreover, $\left.E(L(v)) \cap E_{G}\left(u_{0}\right)\right)=\emptyset$.

Choose $v \in D_{3}^{\prime \prime}(G)$. Since $L(v)$ is a 3-circuit, by Claim 3, $\left|E\left(C_{3}(v)\right) \cap X\right| \geq 1$. By Claim 6, $\left(E\left(C_{3}(v)\right) \cap X\right) \subseteq\left(E(L(v)) \cap E\left(L\left(u_{0}\right)\right)\right)$. By Claim 7, $V(L(v)) \nsubseteq \subseteq\left(L\left(u_{0}\right)\right)$, and then $\left|E(L(v)) \cap E\left(L\left(u_{0}\right)\right)\right| \leq 1$. Thus $\left|E(L(v)) \cap E\left(L\left(u_{0}\right)\right)\right|=|E(L(v)) \cap X|=1$. Since all neighbors of $u_{0}$ are in $\left.L\left(u_{0}\right), E(L(v)) \cap E_{G}\left(u_{0}\right)\right)=\emptyset$. This verifies Claim 11.

Claim 12 For $\forall v \in D_{3}^{\prime \prime}(G)-V\left(L\left(u_{0}\right)\right)$, $v$ has exact one neighbor not in $L\left(u_{0}\right)$. 

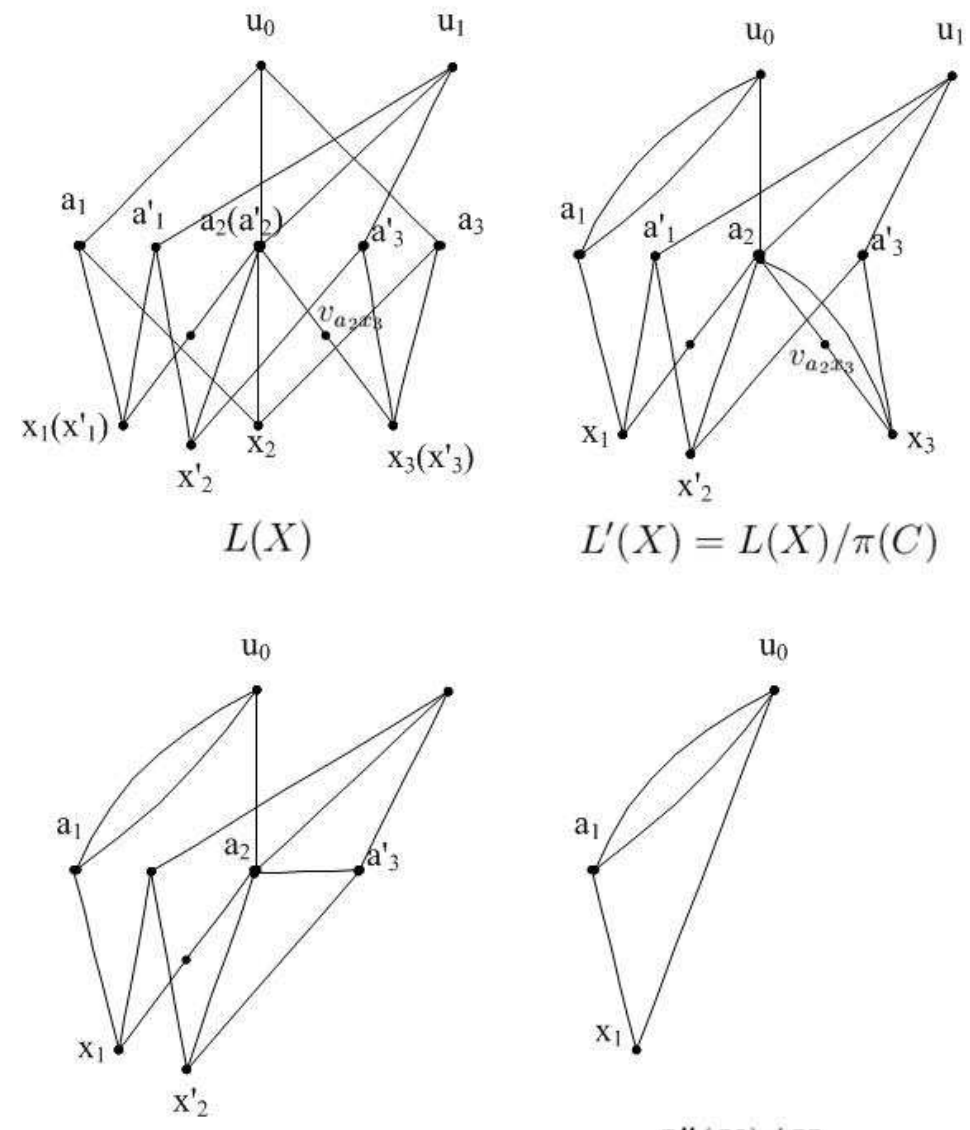

$$
L^{\prime \prime}(X)=L^{\prime}(X) / C^{\prime}
$$$$
L^{\prime \prime}(X) / H
$$

Figure 4.4.11
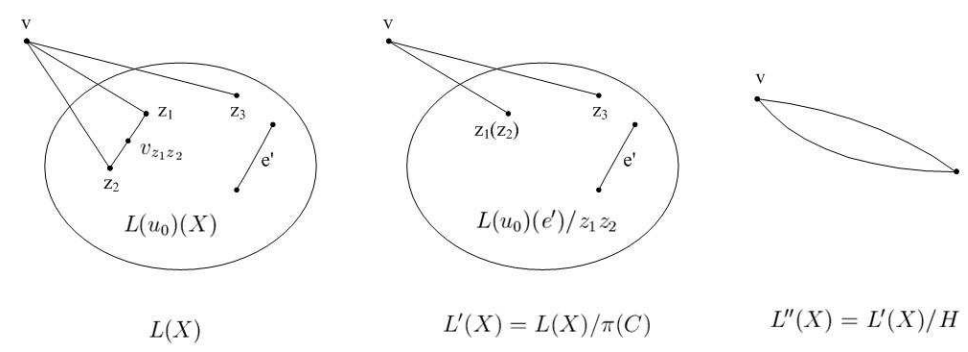

Figure 4.4.12 

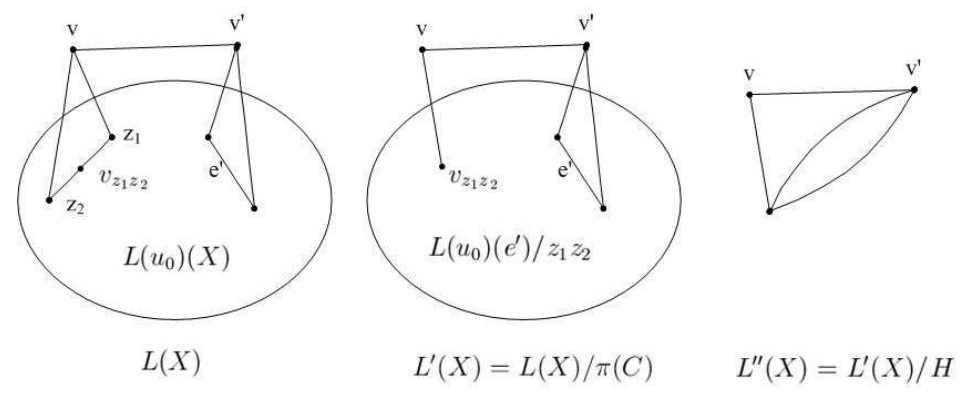

Figure 4.4.13

By Claim 9, for $\forall v \in D_{3}^{\prime \prime}(G)-V\left(L\left(u_{0}\right)\right),\left|N_{G}(v) \cap V\left(L\left(u_{0}\right)\right)\right| \geq 2$. Suppose there exists $v \in D_{3}^{\prime \prime}(G)-V\left(L\left(u_{0}\right)\right)$ such that $N_{G}(v)=\left\{z_{1}, z_{2}, z_{3}\right\} \subseteq V\left(L\left(u_{0}\right)\right)$. Denote $L=L\left(u_{0}\right) \cup$ $E_{G}(v)$ and $L(X)$ is depicted as Fig 4.4.12. Note that $L(v)=v z_{1} z_{2}$ and $X=\left\{z_{1} z_{2}, e^{\prime}\right\}$. Denote $C$ to be the 4 -circuit $v z_{1} v_{z_{1} z_{2}} z_{2}$ in $L(X)$ and denote $L^{\prime}(X)=L(X) / \pi(C)$. Denote $H=L^{\prime}(X)-v$. Then $L^{\prime \prime}(X)=L^{\prime}(X) / H$ is a 2 -circuit, which is collapsible. Note that $H \cong L\left(u_{0}\right)\left(e^{\prime}\right) / z_{1} z_{2}$. By Lemma 4.3 .2 (i) and Theorem 1.2.1 (vi), $\left(L\left(u_{0}\right)(e) / z_{1} z_{2}\right)$ is collapsible, and then $H$ is collapsible. Thus by Theorem 1.2.1 (i) and Theorem 1.2.6 (i), $L^{\prime}(X)$ is collapsible and $L(X)$ is collapsible, a contradiction to Claim 3. This verifies Claim 12.

Claim $13 D_{3}^{\prime \prime}(G)-V\left(L\left(u_{0}\right)\right)$ is an independent set.

By way of contradiction, suppose $v, v^{\prime} \in D_{3}^{\prime \prime}(G)-V\left(L\left(u_{0}\right)\right)$ such that $v v^{\prime} \in E(G)$. Let $N_{G}(v)=\left\{z_{1}, z_{2}, v^{\prime}\right\}$ where $L(v)=v z_{1} z_{2}$ and $X=\left\{z_{1} z_{2}, e^{\prime}\right\}$. If $L\left(v^{\prime}\right) \cap X=\left\{z_{1} z_{2}\right\}$, then $v v^{\prime} z_{1}$ is a triangle in $G(X)$, a contradiction to Claim 3. Thus $L\left(v^{\prime}\right) \cap X=\left\{e^{\prime}\right\}$. Denote $L=L\left(u_{0}\right) \cup E_{G}(v) \cup E_{G}\left(v^{\prime}\right)$ and $L(X)$ is depicted as Fig 4.4.13. Denote $C$ to be the 4-circuit $v z_{1} v_{z_{1} z_{2}} z_{2}$ in $L(X)$ and denote $L^{\prime}(X)=L(X) / \pi(C)$. Denote $H=L^{\prime}(X)-\left\{v, v^{\prime}\right\}$ and denote $L^{\prime \prime}(X)=L^{\prime}(X) / H$. Since $L^{\prime \prime}(X)$ has a spanning 3-circuit, the reduction of $L^{\prime \prime}(X)$ is $K_{1}$, and then $L^{\prime \prime}(X)$ is collapsible. Note that $H \cong L\left(u_{0}\right)\left(e^{\prime}\right) / z_{1} z_{2}$. By Lemma 4.3.2 (i) and Theorem 1.2.1 (vi), $\left(L\left(u_{0}\right)(e) / z_{1} z_{2}\right)$ is collapsible, and then $H$ is collapsible. Thus by Theorem 1.2.1 (i) and Theorem 1.2.6 (i), $L^{\prime}(X)$ is collapsible and $L(X)$ is collapsible, a contradiction to Claim 3. This verifies Claim 13. 


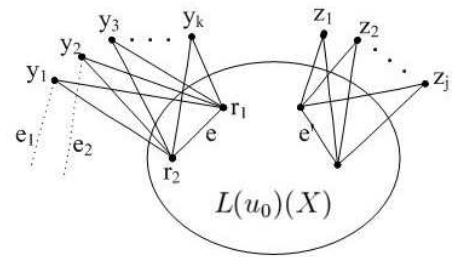

$L(X) \subseteq G(X)$

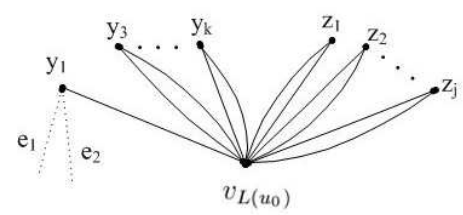

$L^{\prime \prime}(X)=L^{\prime}(X) / H \subseteq G^{\prime \prime}(X)$
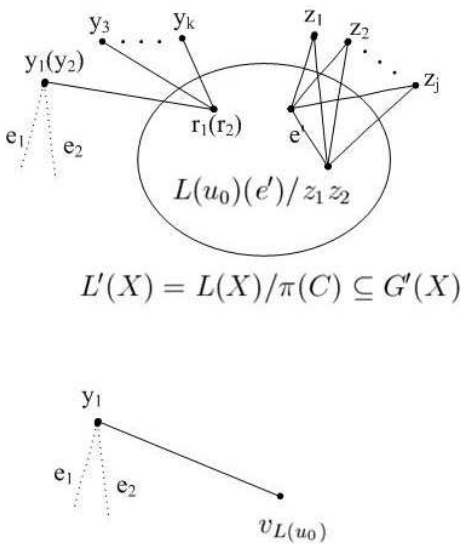

$L^{\prime \prime \prime}(X)=L^{\prime}(X) / H^{\prime} \subseteq G^{\prime \prime \prime}(X)$

Figure 4.4.14

Claim 14 For $\forall e \in X,\left|\left\{v \in D_{3}^{\prime \prime}(G)-V\left(L\left(u_{0}\right)\right): e \in L(v)\right\}\right| \leq 1$. That is, every edge in $X$ is in at most one triangle.

Suppose that $X=\left\{e, e^{\prime}\right\}$. We may assume that $\left\{v \in D_{3}^{\prime \prime}(G)-V\left(L\left(u_{0}\right)\right): e \in\right.$ $L(v)\}=\left\{y_{1}, y_{2}, \ldots, y_{k}\right\}$ and $\left\{v \in D_{3}^{\prime \prime}(G)-V\left(L\left(u_{0}\right)\right): e^{\prime} \in L(v)\right\}=\left\{z_{1}, z_{2}, \ldots, z_{j}\right\}$. By way of contradiction, we may assume that $k \geq 2$. Assume that $r_{1}, r_{2}$ are the endpoints of $e$.

Let $L=L\left(u_{0}\right) \cup\left(\cup_{v \in D_{3}^{\prime \prime}(G)} L(v)\right)$. Note that $D_{3}(G) \subseteq V(L)$ and $X \subseteq E(L)$. Then $G(X) / L$ is 4-edge-connected. By Claim 12 , let $w_{i}$ be the neighbor of $y_{i}(i=1,2)$ that is outside $L\left(u_{0}\right)$. Denote that $e_{i}=y_{i} w_{i}$ for $i=1,2$. By Claim 13, $w_{1}$ and $w_{2}$ are not in $L$ (Fig 4.4.14).

Denote $C$ to be the 4 -circuit $y_{1} r_{1} y_{2} r_{2}$ in $G(X)$. Denote $G^{\prime}(X)=G(X) / \pi(C)$ and denote $L^{\prime}(X)=L(X) / \pi(C)$, which is a subgraph of $G^{\prime}(X)$. Denote $H=L^{\prime}(X)-D_{3}^{\prime \prime}(G)$. Note that $H \cong L\left(u_{0}\right)\left(e^{\prime}\right) / e$. By Lemma 4.3.2 (i) and Theorem 1.2.1 (vi), $H$ is collapsible. Denote $G^{\prime \prime}(X)=G^{\prime}(X) / H$ and $L^{\prime \prime}(X)=L^{\prime}(X) / H$, which is a subgraph of $G^{\prime \prime}(X)$. Denote $H^{\prime}=L^{\prime \prime}(X)-y_{1}$. Since 2 -circuit is collapsible, $H^{\prime}$ is collapsible. Denote $G^{\prime \prime \prime}(X)=$ $G^{\prime \prime}(X) / H^{\prime}$ and $L^{\prime \prime \prime}(X)=L^{\prime \prime}(X) / H^{\prime}$, which is isomorphic to $K_{2}$. Since $G^{\prime \prime \prime}(X) / L^{\prime \prime \prime}(X)$ 
(a)

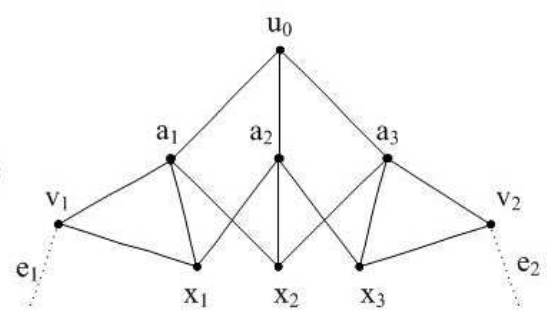

$$
X=\left\{a_{1} x_{1}, a_{3} x_{3}\right\}
$$

(b)

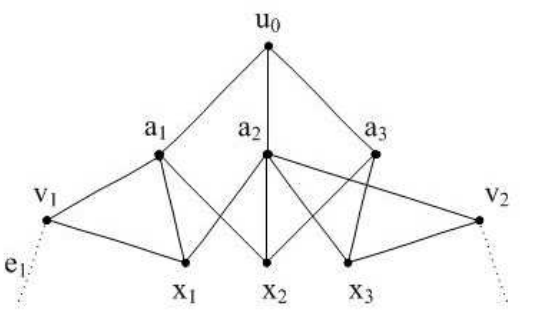

$$
X=\left\{a_{1} x_{1}, a_{2} x_{3}\right\}
$$

(c)

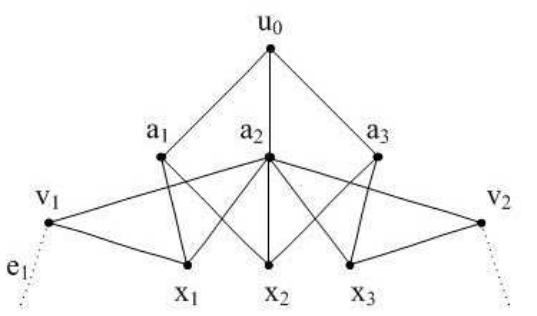

$$
X=\left\{a_{2} x_{1}, a_{2} x_{3}\right\}
$$

Figure 4.4.15

is isomorphic to $G(X) / L, G^{\prime \prime \prime}(X) / L^{\prime \prime \prime}(X)$ is 4-edge-connected. By Theorem 1.2.2 (i), $G^{\prime \prime \prime}(X) / L^{\prime \prime \prime}(X)-\left\{e_{1}, e_{2}\right\}$ has two edge-disjoint spanning trees $T_{1}$ and $T_{2}$. Then $T_{1}+e_{1}$ and $T_{2}+e_{2}$ are two edge-disjoint spanning trees of $G^{\prime \prime \prime}(X)$. Thus $F\left(G^{\prime \prime \prime}(X)\right)=0$ and by Theorem 1.2.4 (ii), $G^{\prime \prime \prime}(X)$ is collapsible. Thus by Theorem 1.2.1 (i) and Theorem 1.2.6 (i), $G^{\prime \prime}(X), G^{\prime}(X)$ and $G(X)$ are all collapsible, a contradiction to Claim 3. This verifies Claim 14.

By Claim 8 and Claim 11, for $\forall e \in X \cap E_{G}\left(u_{0}\right), e$ is not in $L(v)$ for any $v \in D_{3}^{\prime \prime}(G)$; by Claim 8 and Claim 14, for $\forall e \in X-E_{G}\left(u_{0}\right), e$ is in at most one triangle, which contains 


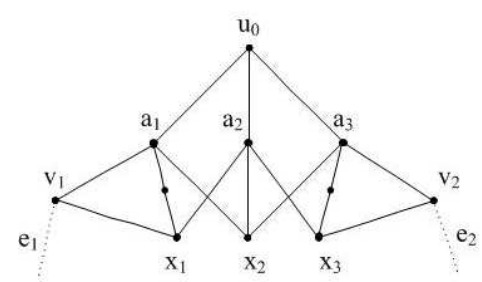

$L(X) \subseteq G(X)$

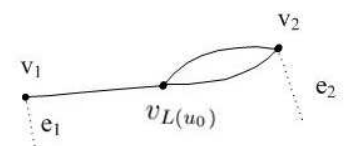

$L^{\prime \prime}(X)=L^{\prime}(X) / H \subseteq G^{\prime \prime}(X)$

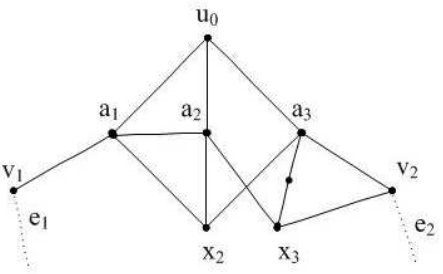

$L^{\prime}(X)=L(X) / \pi(C) \subseteq G^{\prime}(X)$

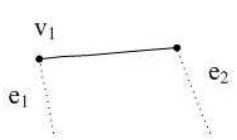

$L^{\prime \prime \prime}(X)=L^{\prime \prime}(X) / C^{\prime} \subseteq G^{\prime \prime \prime}(X)$

Figure 4.4.16

at most two elements of $D_{3}^{\prime \prime}(G)$. Thus by Claim 4 ,

$$
\begin{aligned}
6 & \leq\left|D_{3}(G)\right|=\left|D_{3}^{\prime}(G)\right|+\left|D_{3}^{\prime \prime}(G)\right| \\
& \leq\left|\left\{u_{0}, x_{2}\right\}\right|+2\left|X-E_{G}\left(u_{0}\right)\right| \\
& \leq\left|\left\{u_{0}, x_{2}\right\}\right|+2|X|=6
\end{aligned}
$$

So all equalities must hold, that is $D_{3}^{\prime}(G)=\left\{u_{0}, x_{2}\right\}$ and $X \cap E_{G}\left(u_{0}\right)=\emptyset$. Since $L\left(x_{2}\right)=L\left(u_{2}\right)$, by Claim 11, $X \cap E_{G}\left(x_{2}\right)=\emptyset$. Thus $X \subseteq\left\{a_{1} x_{1}, a_{2} x_{1}, a_{2} x_{3}, a_{3} x_{3}\right\}$. Since $X \notin\left\{\left\{a_{1} x_{1}, a_{2} x_{1}\right\},\left\{a_{2} x_{3}, a_{3} x_{3}\right\}\right\},\left|X \cap\left\{a_{1} x_{1}, a_{2} x_{1}\right\}\right|=\left|X \cap\left\{a_{2} x_{3}, a_{3} x_{3}\right\}\right|=1$. Since each edge $e$ in $X$ is in a unique triangle, which contains two elements of $D_{3}^{\prime \prime}(G)$, then $D_{3}^{\prime \prime}(G)=\left\{x_{1}, x_{3}, v_{1}, v_{2}\right\}$ where $L\left(v_{1}\right)=L\left(x_{1}\right)$ and $L\left(v_{2}\right)=L\left(x_{3}\right)$. Denote $L\left(D_{3}\right)=$ $L\left(u_{0}\right) \cup\left(\cup_{v \in D_{3}^{\prime \prime}(G)} L(v)\right)$. Then $G / L$ is 4-edge-connected. By Claim $11-14, L\left(D_{3}\right)$ has the three possible cases depicted as Fig 4.4.15.

If $X=\left\{a_{1} x_{1}, a_{3} x_{3}\right\}$ (Fig 4.4.15 (a), also Fig 4.4.16), Denote $C$ to be the 4-circuit $a_{1} v_{1} x_{1} v_{a_{1} x_{1}}$ in $G(X)$. Denote $G^{\prime}(X)=G(X) / \pi(C)$ and denote $L^{\prime}(X)=L(X) / \pi(C)$, which is a subgraph of $G^{\prime}(X)$. Denote $H=L^{\prime}(X)-\left\{v_{1}, v_{2}\right\}$. Note that $H \cong L\left(u_{0}\right)\left(a_{3} x_{3}\right) / a_{1} x_{1}$. By Lemma 4.3.2 (i) and Theorem 1.2.1 (vi), $H$ is collapsible. Denote $G^{\prime \prime}(X)=G^{\prime}(X) / H$ and $L^{\prime \prime}(X)=L^{\prime}(X) / H$, which is a subgraph of $G^{\prime \prime}(X)$. Denote $H^{\prime}=L^{\prime \prime}(X)-y_{1}$. S- 
ince 2-circuit is collapsible, $H^{\prime}$ is collapsible. Denote $C^{\prime}$ to be the 2-circuit $v_{L\left(u_{0}\right)} v_{2}$ in $G^{\prime \prime}(X)$. Denote $G^{\prime \prime \prime}(X)=G^{\prime \prime}(X) / C^{\prime}$ and $L^{\prime \prime \prime}(X)=L^{\prime \prime}(X) / C^{\prime}$, which is isomorphic to $K_{2}$. Since $G^{\prime \prime \prime}(X) / L^{\prime \prime \prime}(X)$ is isomorphic to $G(X) / L, G^{\prime \prime \prime}(X) / L^{\prime \prime \prime}(X)$ is 4-edge-connected. By Theorem 1.2.2 (i), $G^{\prime \prime \prime}(X) / L^{\prime \prime \prime}(X)-\left\{e_{1}, e_{2}\right\}$ has two edge-disjoint spanning trees $T_{1}$ and $T_{2}$. Then $T_{1}+e_{1}$ and $T_{2}+e_{2}$ are two edge-disjoint spanning trees of $G^{\prime \prime \prime}(X)$. Thus $F\left(G^{\prime \prime \prime}(X)\right)=0$ and by Theorem 1.2.4 (ii), $G^{\prime \prime \prime}(X)$ is collapsible. Thus by Theorem 1.2.1 (i) and Theorem 1.2.6 (i), $G^{\prime \prime}(X), G^{\prime}(X)$ and $G(X)$ are all collapsible, a contradiction to Claim 3.

If $X=\left\{a_{1} x_{1}, a_{2} x_{3}\right\}$ or $\left\{a_{2} x_{1}, a_{2} x_{3}\right\}$, then by a similar argument we could claim that $G(X)$ is collapsible, a contradiction.

This complete the proof of Theorem 4.4.1.

Proof of Theorem 4.1.7. Assume that $L(G)$ is not complete. By Theorem 1.1.4 (i), $\kappa^{\prime}\left(G_{0}\right) \geq 3$. Since $L(G)$ is 4-connected, $G_{0}$ is essentially 4-edge-connected. By Lemma 4.3.1, for $\forall u \in D_{3}\left(G_{0}\right), u$ lies in a local subgraph $L(u)$ either isomorphic to a contraction of a member in $\left\{L_{1}, L_{2}, L_{3}, L_{4}\right\}$ or $L_{5}$. By Theorem 4.4.1 and Theorem 1.2.1(iii), $G_{0}\left(e^{\prime}, e^{\prime \prime}\right)$ has a spanning $\left(v\left(e^{\prime}\right), v\left(e^{\prime}\right)\right)$-trail for any pair of vertices $e^{\prime}, e^{\prime \prime} \in V\left(G_{0}\right)$. Then by Lemma 4.2.5, $G\left(e^{\prime}, e^{\prime \prime}\right)$ has a dominating $\left(v\left(e^{\prime}\right), v\left(e^{\prime \prime}\right)\right)$-trail, for any $e^{\prime}, e^{\prime \prime} \in E(G)$. By Lemma 4.2.3 and Lemma 4.2.6, Theorem 4.1.7 is proved. 


\section{Chapter 5}

\section{Group Colorability of Multigraphs}

\subsection{Introduction}

Graphs considered in this chapter are multigraphs that are finite and loopless. A nontrivial 2-regular connected graph is called a circuit. Let $G$ be a graph with vertex set $V(G)$ and edge set $E(G)$. We define an equivalence relation " $"$ on $E(G)$ such that $e_{1} \sim e_{2}$ if $e_{1}=e_{2}$, or if $e_{1}$ and $e_{2}$ form a circuit in $G$. Edges in the same equivalence class are parallel edges, and they have the same endpoints. For $u, v \in V(G)$, let $m(u, v)$ denote the number of parallel edges between $u$ and $v$. The multiplicity of $G$, denoted by $M(G)$, is the maximum size of an equivalence class. The simplification of $G$, denoted by $G_{0}$, is the simple graph obtained by replacing each equivalence class by a single edge. For a graph $H$ and a positive integer $k$, we define $k H$ to be the graph obtained by replacing each edge of $H$ by a class of $k$ parallel edges.

Group coloring was first introduced by Jeager et al. [21]. He introduced a concept of group connectivity as a generalization of nowhere-zero flows. He also introduced group coloring as a dual concept to group connectivity.

An orientation $D$ of $G$ is a map $t_{D}: E(G) \rightarrow V(G)$ such that $t_{D}(e)$ is a vertex incident with $e$ in $G$, and the edge $e$ is oriented from $t_{D}(e)$ to the other endpoint. The 
graph $G$ under the orientation $D$ is sometimes denoted by $D(G)$. In $D(G)$, an oriented edge is called an arc, and we write $e=u v$ to mean $t_{D}(e)=u$.

Let $\Gamma$ be a nontrivial group and let $F(G, \Gamma)$ be the set of all functions $f: E(G) \rightarrow \Gamma$. For a function $f \in F(G, \Gamma)$, a $(\Gamma, f)$-coloring of $D(G)$ is a function $c: V(G) \rightarrow \Gamma$ such that $c(u) c(v)^{-1} \neq f(e)$ when $u v$ is an arc; $D(G)$ is $\Gamma$-colorable if and only if for any $f \in F(G, \Gamma)$ there exists a $(\Gamma, f)$-coloring. It is known $([21])$ that whether $G$ is $\Gamma$-colorable is independent of the choice of the orientation. Therefore, we say simply that $G$ is $\Gamma$-colorable when some orientation of $G$ is $\Gamma$-colorable. The group chromatic number of a graph $G$, denoted by $\chi_{g}(G)$, is defined to be the minimum positive integer $m$ for which $G$ is $\Gamma$-colorable for every group $\Gamma$ of order at least $m$.

For a subgraph $H$ of $G,(G, H)$ is said to be $\Gamma$-extendible if for any $f \in F(G, \Gamma)$ and any $\left(\Gamma,\left.f\right|_{E(H)}\right)$-coloring $c^{\prime}$ of $H$, there is a $(\Gamma, f)$-coloring $c$ of $G$ such that $\left.c\right|_{E(H)}=c^{\prime}$. The coloring $c$ is then called an extension of $c^{\prime}$.

Prior research on group chromatic number was restricted to simple graphs, and considered only Abelian groups in the definition of $\chi_{g}(G)$. The following results were under the assumptions that the groups involved are Abelian groups. However, they remain valid without this assumption.

Theorem 5.1.1 (Lai and Zhang, [29]) For any connected simple graph G,

$$
\chi_{g}(G) \leq \Delta(G)+1
$$

with equality if and only if $G$ is a cycle or $G$ is complete.

Theorem 5.1.2 Let $G$ be a simple graph.

(i) (Lai and Zhang, [30]) If $G$ is $K_{5}$-minor free, then $\chi_{g}(G) \leq 5$.

(ii) (Lai and Li, [25]) If $G$ is $K_{3,3}$-minor free, then $\chi_{g}(G) \leq 5$.

The bound of Theorem 5.1.2 (ii) is sharp. A 3-colorable simple planar graph with $\chi_{g}(G)=5$ is constructed in $[22]$. 
Note that although group coloring is a generalization of vertex coloring, they have different behaviors: for any bipartite graph $G, \chi(G)=2$ while $\chi_{g}(G)$ can be arbitrary large. Lai and Zhang [29] showed that for any complete bipartite graph $K_{m, n}$ with $n \geq$ $m^{m}, \chi_{g}\left(K_{m, n}\right)=m+1$. Moreover, group coloring of multigraphs is different from that of simple graphs. Consider $K_{2}$, for an example. By Brooks' Theorem and TM5.1.1, $\chi\left(K_{2}\right)=\chi_{g}\left(K_{2}\right)=2$ while $\chi_{g}\left(m K_{2}\right)=m+1$ for $m \in \mathbf{Z}^{+}$by the definition of $\chi_{g}(G)$.

The main purpose of this note is to extend Theorem 5.1.1 to multigraphs.

Theorem 5.1.3 For any connected multigraph $G$,

$$
\chi_{g}(G) \leq \Delta(G)+1
$$

where equality holds if and only if $G=k C_{n}$ or $k K_{n}$ for some positive integer $k$ and $n$.

In order to prove our theorem, we will first show some properties of group coloring and then introduce the degeneration method. Finally we will give the upper bound of $\chi_{g}(G)$ for multigraphs.

\subsection{Properties of Group Coloring}

In [29], Lai and Zhang proved the following properties of $\chi_{g}$. Although they assumed that graphs are simple and groups are Abelian groups, these properties still hold without such assumptions, as their proofs did not utilize the property that the binary operations of the involved groups are commutative.

Proposition 5.2.1 (Lai and Zhang, [29]) For any simple graph $G, \chi_{g}(G)=2$ if and only if $G$ is a forest.

Proposition 5.2.2 (Lai and Zhang, [29]) If $G$ is a multigraph and $\Gamma$ is a group, then each of the following holds: 
(i) If $G$ is $\Gamma$-colorable under an orientation $D$, then $G$ is $\Gamma$-colorable under every orientation of $G$.

(ii) $G$ is $\Gamma$-colorable if and only if each block of $G$ is $\Gamma$-colorable.

By Proposition 5.2.2 (i), $\chi_{g}(G)$ is not dependent on the choice of orientations of $G$. Furthermore, Proposition 5.2.2 (ii) shows that it suffices to consider 2-connected graphs in our proofs.

Proposition 5.2.3 (Lai and Zhang, [29]) If $G$ is a multigraph and $\Gamma$ is a group, then each of the following holds:

(i) Let $H \subseteq G$. If $(G, H)$ is $\Gamma$-extendible and $H$ is $\Gamma$-colorable, then $G$ is $\Gamma$-colorable.

(ii) Let $H_{2} \subseteq H_{1} \subseteq G$. If $\left(G, H_{1}\right)$ and $\left(H_{1}, H_{2}\right)$ are $\Gamma$-extendible, then $\left(G, H_{2}\right)$ is also $\Gamma$-extendible.

Let $\Gamma$ and $\Gamma^{\prime}$ be two groups and let $\varphi: \Gamma \rightarrow \Gamma^{\prime}$ be a homomorphism. Then $\operatorname{im}(\varphi)$, the image of $\Gamma$ under $\varphi$, is a subgroup of $\Gamma^{\prime}$.

Proposition 5.2.4 (Lai and Zhang, [29]) Let $\varphi: \Gamma \rightarrow \Gamma^{\prime}$ be a homomorphism and $G$ be a multigraph. If $G$ is im $(\varphi)$-colorable, then $G$ is also $\Gamma$-colorable.

Note the fact that: if $N$ is a normal subgroup of $\Gamma$, then the function $\pi: \Gamma \rightarrow$ $\Gamma / N(\Gamma / N$ is called the quotient group of $\Gamma)$ defined by $\pi(a)=a N$ is a homomorphism of $\Gamma$ onto $\Gamma / N$. Also note the fact that if $\Gamma$ and $\Gamma^{\prime}$ are two finite cyclic groups with orders $m$ and $n$, respectively, then there exists a homomorphism of $\Gamma$ onto $\Gamma^{\prime}$ if and only if $n \mid m$. Thus, by Proposition 5.2.4, we have the following result.

Corollary 5.2.5 (Lai and Zhang, [29]) Let G be a multigraph. Then each of the following holds: 
(i) Let $\Gamma$ be a group and $N$ be any subgroup of $\Gamma$. If $G$ is $\Gamma / N$-colorable, then $G$ is $\Gamma$ colorable.

(ii) For any positive integers $k$ and $n$, if $G$ is $Z_{n}$-colorable, then $G$ is also $Z_{k n}$-colorable.

Lemma 5.2.6 If $G$ is a multigraph and $k \in \mathbf{Z}^{+}$, then $\chi_{g}(k G) \geq k\left(\chi_{g}(G)-1\right)+1$.

Proof. By the definition of $\chi_{g}(G)$, there exist a group $\Gamma$ of order $\chi_{g}(G)-1$ and a function $f \in F(G, \Gamma)$ such that there are no $(\Gamma, f)$-colorings of $G$. Let $\Gamma^{\prime}=\Gamma \times \mathbf{Z}_{k}$ (where $\left.\left|\Gamma^{\prime}\right|=k\left(\chi_{g}(G)-1\right)\right)$. Define $f^{\prime} \in F\left(k G, \Gamma^{\prime}\right)$ by $f\left(e_{i}\right)=(f(e), i)$ for $1 \leq i \leq k$ where $\left\{e_{1}, e_{2}, \ldots, e_{k}\right\}$ are the $k$ parallel edges in $E(k G)$ corresponding to $e \in E(G)$. There are no $\left(\Gamma^{\prime}, f^{\prime}\right)$-colorings of $k G$, since any $\left(\Gamma^{\prime}, f^{\prime}\right)$-coloring of $k G$ can give rise to a $(\Gamma, f)$-coloring of $G$. Therefore, $k G$ is not $\Gamma^{\prime}$-colorable, and $\chi_{g}(k G) \geq k\left(\chi_{g}(G)-1\right)+1$.

\subsection{Degeneration Method}

Theorem 5.3.1 Suppose that $V(G)$ can be linearly ordered as $v_{1}, v_{2}, \ldots, v_{n}$ such that $d_{G_{i}}\left(v_{i}\right) \leq k(i=1,2, \ldots, n)$, where $G_{i}=G\left[\left\{v_{1}, v_{2}, \ldots, v_{i}\right\}\right]$. Then for any group $\Gamma$ of order at least $k+1,\left(G_{i+1}, G_{i}\right)(i=1,2, \ldots, n-1)$ is $\Gamma$-extendible and so $G$ is $\Gamma$-colorable.

Such a list of $V(G)$ in Theorem 2.3.1 is called $k$-degenerated. An immediate corollary of Theorem 2.3.1 is given below.

Corollary 5.3.2 (Lai and Zhang, [29]) If $G$ is a multigraph, then each of the following holds:

(i) $\chi_{g}(G) \leq \max _{H \subseteq G}\{\delta(H)\}+1$.

(ii) $\chi_{g}(G) \leq \Delta(G)+1$. 
Since every nontrivial simple graph without a subdivision of $K_{4}$ has a vertex of degree at most 2, by Proposition 5.2.1 and Theorem 2.3.1, we have the following result:

Corollary 5.3.3 (Lai and Zhang, [29]) Let $G$ be a nontrivial simple graph without subdivision of $K_{4}$. Then $\chi_{g}(G)=3$ if and only if $G$ has a cycle.

\subsection{Group Chromatic Number of Multigraphs}

Brooks proved that for any connected graph $G, \chi(G) \leq \Delta(G)+1$ where equality holds if and only if $G$ is an odd cycle or a complete graph. Lai and Zhang [29] proved Theorem 5.1.1 as a strengthening of Brooks' Theorem for the group chromatic number of simple graphs. In this section, we shall extend Theorem 5.1.1 to Theorem 5.1.3. We start with two lemmas.

Lemma 5.4.1 Let $\Gamma$ be a group. If $S_{1}$ and $S_{2}$ are subsets of $\Gamma$ such that $\left|S_{2}\right|>\left|S_{1}\right|$, then there exist $x$ and $y$ in $S_{2}$ such that $S_{1} x \neq S_{1} y$.

Proof. We argue by contradiction and assume that $\forall x, y \in S_{2}, S_{1} x=S_{1} y$. Let $\left|S_{1}\right|=m$. Since $\left|S_{2}\right|>\left|S_{1}\right|$, we can pick $m+1$ distinct elements $b_{1}, b_{2}, \ldots, b_{m+1}$ from $S_{2}$. If $1 \leq$ $i<j \leq m+1$, then $S_{1} b_{i}=S_{1} b_{j}$. Fix $a \in S_{1}$. Since $a b_{i} \in S_{1} b_{i}=S_{1} b_{1}(1 \leq i \leq m+1)$, $\left\{a b_{i}: 1 \leq i \leq m+1\right\} \subseteq S_{1} b_{1}$. Since $m=\left|S_{1} b_{1}\right| \geq\left|\left\{a b_{i}: 1 \leq i \leq m+1\right\}\right|$, there exist $1 \leq k<l \leq m+1$ such that $a b_{k}=a b_{l}$. Hence $b_{k}=b_{l}$, contrary to the assumption that $b_{1}, b_{2}, \ldots, b_{m+1}$ are distinct.

Lemma 5.4.2 If $G$ is a 2-connected graph whose simplification is neither a cycle nor a complete graph, then there exist three vertices $v_{1}, v_{2}, v_{n}$ in $G$ such that both of the following holds:

(i) $v_{1} v_{n}, v_{2} v_{n} \in E(G)$ and $v_{1} v_{2} \notin E(G)$, and

(ii) $G-\left\{v_{1}, v_{2}\right\}$ is connected. 
Proof. An ordered triple $\left(v_{1}, v_{2}, v_{n}\right)$ is called "good" if it satisfies both (i) and (ii). We argue by contradiction and assume that $G$ is a counterexample. So there are no good ordered triples in $V(G)$. Since $G$ and its simplification $G_{0}$ have the same vertex connectivity, without loss of generality, we may assume that $G$ is simple. Let $C=$ $v_{1} v_{2} \ldots v_{k}$ be a longest circuit in $G$.

If $C$ is not a Hamilton circuit of $G$, let $H$ be one component of $G-C$. Let $V_{1}=$ $\left\{v_{i} \in V(C): v_{i} u \in E(G), u \in V(H)\right\}$. Since $G$ is 2-connected, then $\left|V_{1}\right| \geq 2$. We can assume that $v_{1} \in V_{1}$, and $v_{1} u_{1} \in E(G)$ where $u_{1} \in V(H)$. Then $v_{2} \notin V_{1}$ by the choice of $C$. Therefore $\left(u_{1}, v_{2}, v_{1}\right)$ is good, a contradiction to the choice of $G$.

So $C$ must be a Hamilton circuit of $G$. Since $G=G_{0}$ is not a cycle, $C$ must have a chord, say $v_{1} v_{i}$. Then $v_{2} v_{k} \in E(G)$, otherwise $\left(v_{2}, v_{k}, v_{1}\right)$ is a good triple. Since $\left(v_{1}, v_{3}, v_{2}\right)$ is not a good triple, $v_{1} v_{3} \in E(G)$. Inductively, since $\left(v_{1}, v_{j}, v_{j-1}\right)$ is not a good triple(for $j=4,5, \cdots, i-1), v_{1} v_{j} \in E(G)$. Therefore $v_{1}$ is adjacent to all vertices of $\left\{v_{2}, v_{3}, \cdots, v_{i}\right\}$. Similarly, $v_{1}$ is adjacent to all vertices of $\left\{v_{i+1}, v_{i+2}, \cdots, v_{k}\right\}$. That is $v_{1}$ is adjacent to every other vertex of $G$. Since $G=G_{0}$ is not a complete graph, there exist $v_{i_{1}}$ and $v_{i_{2}}$ such that $v_{i_{1}} v_{i_{2}} \notin E(G)$. Thus $\left(v_{i_{1}}, v_{i_{2}}, v_{1}\right)$ is a good triple, a contradiction to the choice of $G$.

Proof of Theorem 5.1.3 By Corollary 5.3.2 (ii), we only need to show that when the equality holds, $G$ must be either a $k C_{n}$ or a $k K_{n}$ for some positive integer $k$ and $n$. Let $G$ be a multigraph such that $\chi_{g}(G)=\Delta(G)+1$ and $n=|V(G)|$. By Propsition 5.2.2 (ii), we may assume that $G$ is 2-connected.

Claim $1 G$ is regular.

If $G$ not regular, then $\max _{H \subseteq G}\{\delta(H)\} \leq \Delta(G)-1$. Therefore, by Corollary 5.3.2 (i), $\chi_{g}(G) \leq \max _{H \subseteq G}\{\delta(H)\}+1 \leq \Delta(G)$, a contradiction to $\chi_{g}(G)=\Delta(G)+1$. 
Claim $2 G_{0}$ is either a cycle or a complete graph.

Suppose that $G_{0}$ is neither a cycle nor a complete graph. Since $\chi_{g}(G)=\Delta(G)+1$, there exist a group $\Gamma$ of order $\Delta(G)$ and a function $f \in F(G, \Gamma)$ such that $G$ has no $(\Gamma, f)$-colorings.

By Lemma 5.4.2, $G$ has three vertices $v_{1}, v_{2}, v_{n}$ such that both $v_{1} v_{n}, v_{2} v_{n} \in E(G)$ and $v_{1} v_{2} \notin E(G)$, and such that $G-\left\{v_{1}, v_{2}\right\}$ is connected. Now we arrange the vertices of $G-\left\{v_{1}, v_{2}\right\}$ in non-increasing order of their distance from $v_{n}$, say $v_{3}, \ldots, v_{n}$. Then the list $\left\{v_{1}, v_{2}, \cdots, v_{n}\right\}$ is such that each vertex other than $v_{n}$ is adjacent to at least one vertex following it. Thus each vertex other than $v_{n}$ is adjacent to at most $\Delta(G)-1$ vertices preceding it.

Let $D$ be an orientation such that every arc between $v_{i}$ and $v_{j}$ is directed from $v_{j}$ to $v_{i}$ if $i<j$ and from $v_{i}$ to $v_{j}$ otherwise. Define a map $c: V(G) \rightarrow \Gamma$ as follows. For $i=1,2$, let $e_{i}$ denote an arc from $v_{n}$ to $v_{i}$. Choose $a_{1}, a_{2} \in \Gamma$ such that $f\left(e_{1}\right) a_{1}=f\left(e_{2}\right) a_{2}$. Define $c\left(v_{i}\right)=a_{i}(i=1,2)$. For $v_{j}(3 \leq j \leq n)$, let $A_{j}=\left\{f(e) c\left(v_{i}\right): e \in E\left[v_{j}, v_{i}\right]\right.$ and $i=$ $1,2, \cdots, j-1\}$. If $3 \leq j<n$, then $\left|A_{j}\right| \leq d_{G\left[\left\{v_{1}, v_{2}, \cdots, v_{j}\right\}\right]}\left(v_{j}\right) \leq \Delta(G)-1$ and $\Gamma-A_{j} \neq \emptyset$; if $j=n$, then $\Gamma-A_{n} \neq \emptyset$ since $f\left(e_{1}\right) a_{1}=f\left(e_{2}\right) a_{2}$. Hence we can choose $c\left(v_{j}\right) \in \Gamma-A_{j}$ $(3 \leq j \leq n)$, so that $c$ is a $(\Gamma, f)$-coloring of $G$, contrary to the assumption that $G$ has no $(\Gamma, f)$-colorings.

Claim 3 If $G=k C_{n}$ or $k K_{n}$, then $\chi_{g}(G)=\Delta(G)+1$.

If $G=k C_{n}$, then by Corollary 5.3.2 (ii) and Lemma 5.2.6, $\Delta(G)+1 \geq \chi_{g}(G) \geq$ $k(3-1)+1=\Delta(G)+1$, and so $\chi_{g}(G)=\Delta(G)+1$. Similarly, if $G=k K_{n}$, then $\chi_{g}(G)=\Delta(G)+1$.

Claim 4 If $G_{0}=C_{n}$, then $G=k C_{n}$, where $k=M(G)$. 
Assume that $G \neq k C_{n}$. By Claim 1, $G$ is regular. It follows that $G$ must satisfy both $G_{0}=C_{2 k}=u_{1} v_{1} u_{2} v_{2} \ldots u_{k} v_{k}$, and $m\left(u_{i} v_{i}\right)=a, m\left(v_{i} u_{i+1}\right)=b$ with $a \neq b$, where $i=1,2, \cdots, k$ and where the subscripts are taken modulo $k$. Without loss of generality, assume $a>b$. Let $D$ be an orientation of $G$ such that every $\operatorname{arc} e \in E\left[u_{i}, v_{i}\right]$ is directed from $u_{i}$ to $v_{i}$ and every arc $e \in E\left[v_{i}, u_{i+1}\right]$ is directed from $v_{i}$ to $u_{i+1}(i=1,2, \cdots, k$, where subscripts are taken modulo $k$ ).

Since $\chi_{g}(G)=\Delta(G)+1$, there exist a group $\Gamma$ of order $\Delta(G)=a+b$ and a function $f \in$ $F(G, \Gamma)$ such that $G$ has no $(\Gamma, f)$-colorings. Let $H=G\left[\left\{u_{1}, v_{1}, u_{2}, v_{2}, \ldots, u_{k-1}, v_{k-1}\right\}\right]$. Since $H_{0}$ is a path, it follows by Corollary 5.3.2 that $\chi_{g}(H) \leq \Delta(H)=a+b$ and then $H$ has a $\left(\Gamma,\left.f\right|_{H}\right)$-coloring $c^{\prime}$. Let $c: V(G) \rightarrow \Gamma$ be a function where $\left.c\right|_{V(H)}=c^{\prime}$.

Let $\Gamma_{1}=\Gamma \backslash\left\{f(e): e \in E\left[v_{k-1}, u_{k}\right]\right\}$ and $\Gamma_{2}=\left\{f(e): e \in E\left[v_{k}, u_{1}\right]\right\}$. Pick $y_{0} \in$ $\Gamma \backslash\left\{f(e): e \in E\left[u_{k}, v_{k}\right]\right\}$. Since $\left|\left\{y_{0}^{-1} x^{-1} c\left(v_{k-1}\right) c\left(u_{1}\right)^{-1}: x \in \Gamma_{1}\right\}\right|=\left|\Gamma_{1}\right| \geq a>b \geq$ $\left|\Gamma_{2}\right|$, choose $x_{0} \in \Gamma_{1}$ such that $y_{0}^{-1} x_{0}^{-1} c\left(v_{k-1}\right) c\left(u_{1}\right)^{-1} \notin \Gamma_{2}$. Let $c\left(u_{k}\right)=x_{0}^{-1} c\left(v_{k-1}\right)$ and $c\left(v_{k}\right)=y_{0}^{-1} c\left(u_{k}\right)$. Now $c$ is a $(\Gamma, f)$-coloring of $G$, contrary to the assumption that $G$ has no $(\Gamma, f)$-colorings. This completes the proof of Claim 4.

Claim 5 If $G_{0}=K_{n}$, then $G=k K_{n}$, where $k=M(G)$.

Assume that $G \neq k K_{n}$ and $n \geq 4$. Since $\chi_{g}(G)=\Delta(G)+1$, there exist a group $\Gamma$ of order $\Delta(G)$ and a function $f \in F(G, \Gamma)$ such that there are no $(\Gamma, f)$-colorings of $G$. By Claims $1, G$ is regular. It follows that there exist $u, v_{1}, v_{2} \in V(G)$ with $m\left(u v_{1}\right)=a$, $m\left(u v_{2}\right)=b$ and $m\left(v_{1} v_{2}\right)=d$ such that $a<b$. Let $H=G-\left\{u, v_{1}, v_{2}\right\}$. Let $D$ be such an orientation that arcs in $E\left[\left\{u, v_{1}, v_{2}\right\}, V(H)\right]$ are all directed into $H$; arcs in $E\left[u, v_{i}\right]$ are all directed from $u$ to $v_{i}(i=1,2)$ and arcs in $E\left[v_{2}, v_{1}\right]$ are all directed from $v_{2}$ to $v_{1}$.

Since $H$ is not regular, it follows by Corollary ??(i) that $\chi_{g}(H) \leq \Delta(H) \leq \Delta(G)$. Thus $H$ has a $\left(\Gamma,\left.f\right|_{H}\right)$-coloring $c$. For any $v \in\left\{v_{1}, v_{2}, u\right\}$, define $A_{v}=\Gamma \backslash\{f(e) c(x): x \in$ $V(H), e \in E[v, x]\}$. Since $|\Gamma|=\Delta(G),\left|A_{v_{1}}\right| \geq a+d,\left|A_{v_{2}}\right| \geq b+d$, and $\left|A_{u}\right| \geq a+b$. Taking a subset if needed, we may assume that $\left|A_{v_{1}}\right|=a+d,\left|A_{v_{2}}\right|=b+d$, and $\left|A_{u}\right|=a+b$. Since $\left|\left\{f(e): e \in E\left[v_{2}, v_{1}\right]\right\}\right| \leq d$ and $\left|A_{v_{1}}\right|=a+d$, it follows by Lemma 5.4.1 that there 
exist $x_{1}, x_{2} \in A_{v_{1}}$ such that $\left\{f(e) x_{1}: e \in E\left[v_{2}, v_{1}\right]\right\} \neq\left\{f(e) x_{2}: e \in E\left[v_{2}, v_{1}\right]\right\}$.

Let $c_{1}$ be an extension of $c$ on $G\left[V(H) \cup v_{1}\right]$ such that $c_{1}\left(v_{1}\right)=x_{1}$. For any $v \in\left\{v_{2}, u\right\}$, define $A_{v}^{\prime}=A_{v} \backslash\left\{f(e) x_{1}: e \in E\left[v, v_{1}\right]\right\}$. Note that $\left|A_{v_{2}}^{\prime}\right| \geq b$ and $\left|A_{u}^{\prime}\right| \geq b$. If $\left|A_{v_{2}}^{\prime}\right|>b$, then choose $c_{1}(u) \in A_{u}^{\prime}$ and $c_{1}\left(v_{2}\right) \in A_{v_{2}}^{\prime} \backslash\left\{f(e)^{-1} c_{1}(u)\right\}$, and now $c_{1}$ is a $(\Gamma, f)$-coloring of $G$, contrary to the assumption that $G$ has no $(\Gamma, f)$-colorings. Thus

$$
\left|A_{v_{2}}^{\prime}\right|=b \text {, and similarly }\left|A_{u}^{\prime}\right|=b \text {. }
$$

Assume that there is a $z \in A_{u}^{\prime}$ such that $\left\{f(e)^{-1} z: e \in E\left[u, v_{2}\right]\right\} \neq A_{v_{2}}^{\prime}$. Since $\left|A_{v_{2}}^{\prime}\right|=$ $b=m\left(u v_{2}\right) \geq\left|\left\{f(e)^{-1} z: e \in E\left[u, v_{2}\right]\right\}\right|$, we can pick $y \in A_{v_{2}}^{\prime} \backslash\left\{f(e)^{-1} z: e \in E\left[u, v_{2}\right]\right\}$ and extend $c_{1}$ to a map $c_{2}: V(G) \mapsto \Gamma$ by assigning $c_{2}\left(v_{2}\right)=y$ and $c_{2}(u)=z$. By the choices of $y$ and $z$, it is routine to verify that $c_{2}$ is indeed a $(\Gamma, f)$-coloring of $G$, contrary to the assumption that $G$ has no $(\Gamma, f)$-colorings. Hence we may assume that

$$
\forall z \in A_{u}^{\prime},\left\{f(e)^{-1} z: e \in E\left[u, v_{2}\right]\right\}=A_{v_{2}}^{\prime} .
$$

Let $c_{0}$ be an extension of $c$ on $G$ such that $c_{0}\left(v_{1}\right)=x_{2}$. For any $v \in\left\{v_{2}, u\right\}$, define $A_{v}^{\prime \prime}=A_{v} \backslash\left\{f(e) x_{2}: e \in E\left[v, v_{1}\right]\right\}$. By the choice of $x_{1}$ and $x_{2}, A_{v_{2}}^{\prime} \neq A_{v_{2}}^{\prime \prime}$. So we can pick $y_{0} \in A_{v_{2}}^{\prime \prime} \backslash A_{v_{2}}^{\prime}$. As $G$ has no $(\Gamma, f)$-colorings, it follows by a a similar argument to conclude (1) that we must also have $\left|A_{v_{2}}^{\prime \prime}\right|=\left|A_{u}^{\prime \prime}\right|=b$.

Since $\left|A_{u}\right|=a+b \leq 2 b, A_{u}^{\prime \prime} \cap A_{u}^{\prime} \neq \emptyset$. Take $z_{0} \in A_{u}^{\prime \prime} \cap A_{u}^{\prime}$. Define $c_{2}\left(v_{2}\right)=y_{0}$ and $c_{2}(u)=z_{0}$. By $(2)$ and by the fact that $y_{0} \notin A_{v_{2}}^{\prime}$, it is routine to verify that $c_{0}$ is indeed a $(\Gamma, f)$-coloring of $G$, contrary to the assumption that $G$ has no $(\Gamma, f)$-colorings. This completes the proof of Claim 5.

After we have established these claims, it is straightforward to see that Theorem 5.1.3 now follows from Claims 3, 4 and 5 .

Since $\Delta(G) \leq M(G) \Delta\left(G_{0}\right)$, Corollary 3.3 below follows from Theorem 5.1.3 immediately.

Corollary 5.4.3 For any graph $G, \chi_{g}(G) \leq M(G) \Delta\left(G_{0}\right)+1$ with equality if and only if $G=M(G) C_{n}$ or $G=M(G) K_{n}$. 


\section{Bibliography}

[1] A. Ainouche, Quasi claw-free graphs, Discrete Math. 179 (1998), 13-26.

[2] L. Beineke, Derived graphs and digraphs Beiträge zur Graphentheorie, Teubner, Leipzig, 1968.

[3] F. T. Boesch, C. Suffel, and R. Tindell, The spanning subgraphs of eulerian graphs, J. Graph Theory, 1 (1977) 79-84.

[4] J. A. Bondy and U. S. R. Murty, Graph Theory. Springer, New York, 2008.

[5] H. J. Broersma, and E. Vumer, On Hamiltonicity of $P_{3}$-dominating graphs, Math. Meth. Oper. Res. 69 (2009), 297-306.

[6] P. A. Catlin, Super-Eulerian graphscollapsible graphs, and four-cycles, Congr. Numer. 58 (1987) 233-246.

[7] P. A. Catlin, A reduction method to find spanning eulerian subgraphs, J. Graph Theory 12 (1988) 29-45.

[8] P. A. Catlin, Super-Eulerian graphs, a survey, J. Graph Theory, 16 (1992) 177-196.

[9] P. A. Catlin, The reduction of graph families closed under contraction, Discrete Math. 160 (1996), 67-80.

[10] P. A. Catlin, Z. Han and H-J. Lai, Graphs without spanning eulerian subgraphs, Discrete Math., 160 (1996) 81-91. 
[11] P. A. Catlin, A. M. Hobbs and H.-J. Lai, Operations and graph families, Discrete Math. 230 (2001), 71-98.

[12] P. A. Catlin and H.-J. Lai, Spanning trails joining two given edges, "Graph theory, combinatorics, and applications", eds. by Y. Alavi, G. Chartrand, O. R. Ollermann, A. J. Schwenk, John Wiley and Sons, Inc. (1991), 207-222.

[13] Z. H. Chen and H.-J. Lai, Reduction techniques for super-Eulerian graphs and related topics (a survey), Combinatorics and graph theory 95, Vol. 1 (Hefei), pp. 53-69, World Sci. Publishing, River Edge, NJ, 1995.

[14] Z. H. Chen, H.-J. Lai, H. Y. Lai and G. Weng, Jackson's conjecture on eulerian subgraphs, Combinatorics, Graph Theory, Algorithms and Applications, (eds. by Y. Alavi et al), 53-58, World Scientific, River Edge, NJ (1994).

[15] R. Gould, Advances on the Hamiltonian Problem - A Survey, Graphs and Combinatorics, 19 (2003) 7-52.

[16] R. Halin: Uber einen satz von K. Wagner zum vierfarbenproblem. Math. Ann, 153, 47-62 (1964).

[17] F. Harary, Graph Theory, Edison-Wesley Publishing Company, Reading, (1969).

[18] F. Harary and C. St. J. A. Nash-Williams, On eulerian and hamiltonian graphs and line graphs, Canad. Math. Bull. 8 (1965), 701-709.

[19] F. Harary and W. Tutte: A dual form of Kuratowski's Theorem. Can. Math. Bull, 8, 17-20 (1965).

[20] F. Jaeger, A note on subeulerian graphs, J. Graph Theory 3 (1979) 91-93.

[21] F. Jaeger, N. Linial, C. Payan, and M. Tarsi, Graph Connectivity of Graphs-A Nonhomogeneous Analogue of Nowhere-Zero Flow Properties, J. Combin. Theory Series B 56(1992), 165-182.

[22] D. Král, O. Pangrác and H.-J. Voss, A note on group colorings, J. Graph Theory 50(2005), 123-129. 
[23] M. Kriesell, All 4-Connected Line Graphs of Claw Free Graphs Are HamiltonianConnected. J. Combin. Theory Ser. B 82 (2001), 306-315.

[24] H.-J. Lai, Graphs whose edges are in small cycles. Disc. Math., 94(1991) 11-22.

[25] H.-J. Lai and X. Li, Group chromatic number of graph. Graphs and Combinatorics, $21(2005)$ 469-474.

[26] H.-J. Lai, X. Li, Y. Ou and H. Poon, Spanning trails joining given edges, Graphs and Combinatorics, 21 (2005) 77-88.

[27] H.-J. Lai, Y. H. Shao, G. Yu and M. Zhan, Hamiltonian connectedness in 3-connected line graphs, Discrete Applied Math. 157 (2009), no. 5, 982-990.

[28] H.-J. Lai, Y. H. Shao and M. Zhan, Every 4-connected line graph of a quasi claw-free graph is Hamiltonian connected, Discrete Math., 308 (2008) 5312-5316.

[29] H.-J. Lai and X. Zhang: Group colorability of graphs. Ars Combinatoria, 62, 299-317 (2002).

[30] H.-J. Lai and X. Zhang: Group chromatic number of graphs without $K_{5}$-minors. Graphs and Combinatorics, 18, 147-154 (2002).

[31] M. M. Matthews and D. P. Sumner, Hamiltonian results in $K_{1,3}$-free graphs, J. Graph Theory, 8, (1984), 139-146.

[32] W. R. Pulleyblank, A note on graphs spanned by eulerian graphs. J. Graph Theory 3 (1979) 309-310.

[33] N. Robertson, unpublished notes.

[34] Z. Ryjáček, Hamiltonian circuits in $N_{2}$-locally connected $K_{1,3}$-free graphs, J. Graph Theory, 14 (1990), 321-331.

[35] Z. Ryjáček, On a closure concept in claw-free graphs, J. Combin. Theory Ser. B, 70 (1997), 217-224.

[36] Z. Ryjáček, Almost claw-free graphs, J. Graph Theory, 18 (2006), 469-477. 
[37] Z. Ryjáček and P. Vrána, Line graphs of multigraphs and Hamiltonian-connectedness of claw-free graphs, to appear.

[38] Y. Shao, Claw-free graphs and line graphs, Ph. D. Dissertation, West Virginia University, (2005).

[39] R. Škrekovski: Choosability of $K_{5}$-minor free graphs. Discrete Math, 190, 223-226 (1998).

[40] C. Thomassen, Reflections on graph theory, J. Graph Theory, 10 (1986), 309-324.

[41] C. Thomassen: Every planar graph is 5-choosable. J. Comb. Theory, Ser. B, 62, 180-181 (1994).

[42] K. Wagner, Uber eine eigneschaft der ebenen komplexe, Math. Ann, 144, 570-590 (1937).

[43] S. Zhan, Hamiltonian connectedness of line graphs. Ars Combinatoria, 22 (1986), 89-95.

[44] S. M. Zhan, On Hamiltonian line graphs and connectivity, Discrete Math. 89 (1991) 89-95. 